\title{
Design considerations for variable power split hydraulic drives for industrial applications
}

\author{
Michael Jan Christian Shellenberger \\ West Virginia University
}

Follow this and additional works at: https://researchrepository.wvu.edu/etd

\section{Recommended Citation}

Shellenberger, Michael Jan Christian, "Design considerations for variable power split hydraulic drives for industrial applications" (1999). Graduate Theses, Dissertations, and Problem Reports. 956.

https://researchrepository.wvu.edu/etd/956

This Thesis is protected by copyright and/or related rights. It has been brought to you by the The Research Repository @ WVU with permission from the rights-holder(s). You are free to use this Thesis in any way that is permitted by the copyright and related rights legislation that applies to your use. For other uses you must obtain permission from the rights-holder(s) directly, unless additional rights are indicated by a Creative Commons license in the record and/ or on the work itself. This Thesis has been accepted for inclusion in WVU Graduate Theses, Dissertations, and Problem Reports collection by an authorized administrator of The Research Repository @ WVU. For more information, please contact researchrepository@mail.wvu.edu. 


\title{
Design Considerations for Variable Power Split Hydraulic Drives for Industrial Applications
}

\author{
By \\ Michael J.C. Shellenberger
}

\begin{abstract}
A THESIS
Submitted to the College of Engineering and Mineral Resources at West Virginia University
\end{abstract}

in partial fulfillment of the requirements

for the degree of

Master of Science in

Mechanical Engineering

Department of Mechanical and Aerospace Engineering

Morgantown, West Virginia

1999

Committee Chair: Victor H. Mucino, Ph. D.

Kenneth H. Means, Ph. D.

W. Scott Wayne, Ph. D. 


\section{Abstract}

Continuously variable transmissions (CVTs) offer some advantages over conventional transmissions in industrial applications. During discrete shifts, conventional transmissions suffer from momentary power transmission lapses, which may produce undesirable effects in the system. Currently, the types of CVTs utilized in industry are hydrostatic drives and hydrodynamic couplings. However, these drives are heavy, presenting an obstacle in applications where weight is a concern. In a power split continuously variable transmission (PS-CVT) the power that flows through the hydraulic element is reduced. This allows the overall size and weight of the transmission to be reduced.

The objective of this thesis is to develop a conceptual design procedure for development of a power split hydraulic variable transmission to be used in industrial applications. The procedure consists of a 15 step process which is outlined by means of a flow chart. The results of the procedure can be used to estimate the overall size and efficiencies of the conceptual transmission and to produce $2 \mathrm{D}$ and $3 \mathrm{D}$ scale drawings of the design. This procedure offers a means to improve the efficiency of the conceptual design stage of a PS-CVT.

An industrial pump was used as an example application to illustrate the conceptual design procedure. Two conceptual designs were completed utilizing different hydraulic variable elements. The two variable elements considered were a hydrostatic drive and a hydrodynamic coupling. The two examples illustrated the procedure that can be used to determine whether a PS-CVT is a viable alternative for an industrial pumping system. 


\section{Acknowledgements}

I would like to take this opportunity to express my thanks to all of the people that helped make my graduate studies at West Virginia University successful. First of all I would like to thank my research advisor, Dr. Victor H. Mucino, for his guidance and patience and for the intellectual challenges that provided me with a great deal of inspiration throughout my research. Thanks also to my remaining committee members, Dr. Kenneth H. Means and Dr. W. Scott Wayne, for their continuous guidance throughout this project.

My thanks also to Stan Stephenson and Lewis Norman at Halliburton Energy Services for their support and input during my research.

I would also like to thank Michael W. McWhorter and David Morris for their guidance while I was in the process of writing this thesis.

Last, but definitely not least, I would like to thank the three most important people in my life, my mother and father, Shirley and Ronald Shellenberger, and my fiancé Traci Curry. Thanks mom and dad for your unending love and support through seven long years of college. And thanks to Traci for putting up with me and your support, understanding, encouragement and love throughout my time here at graduate school. 


\section{Table of Contents}

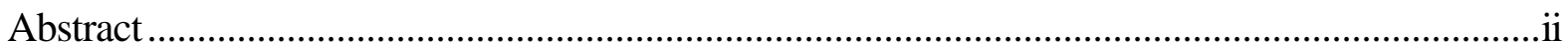

Acknowledgements ...................................................................................................................ii

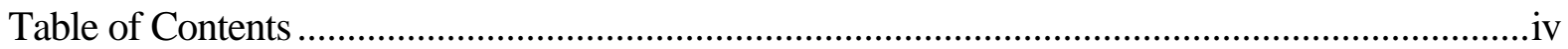

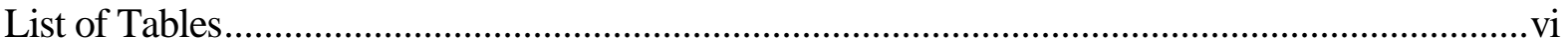

List of Figures ...........................................................................................................

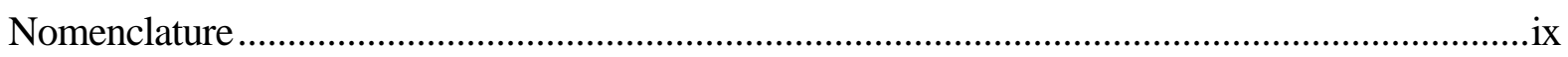

Chapter 1 Introduction ........................................................................................................ ix

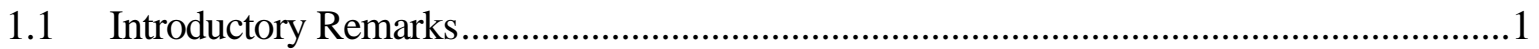

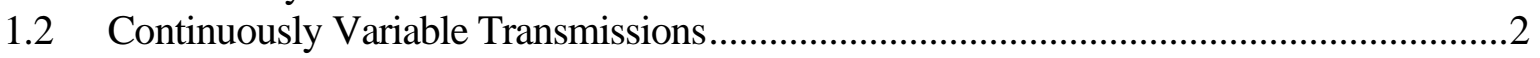

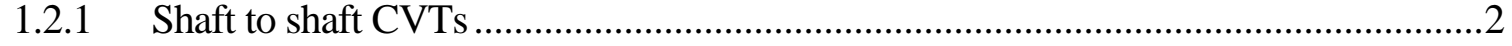

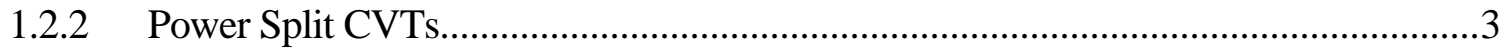

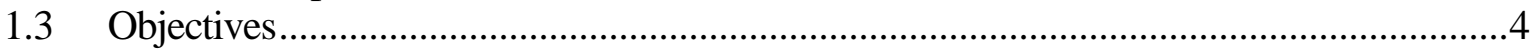

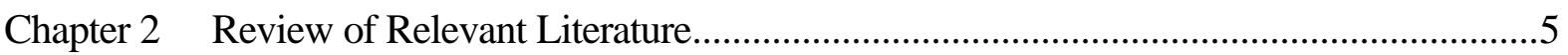

2.1 Power Split Continuously Variable Transmission Configurations ......................................5

2.2 Extended range PS-CVTs ....................................................................................

2.3 PS-CVT Design for Applications ................................................................................

Chapter 3 PS-CVT Concepts and Components ………………………………………….......12

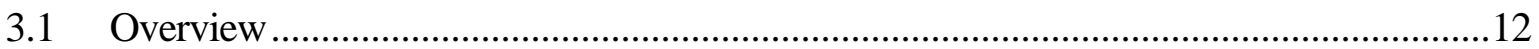

3.2 Input and Output Configuration Gear Arrangements ..................................................12

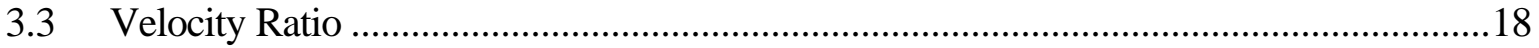

3.3.1 Analysis of the Velocity Ratio using Kinematics ...................................................... 18

3.3.2 Analysis of Velocity Ratio using Force Analysis .....................................................21

3.4 Velocity Ratio Curve and Span of Velocity Ratios ..........................................................2

3.5 Power Flow through Variable Element ..................................................................26

3.5.1 Calculation of Gamma in the Ideal Case ………..................................................26

3.5.2 Calculation of Gamma with Power Loss ..................................................................31

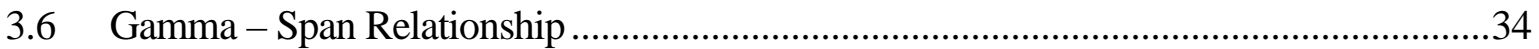

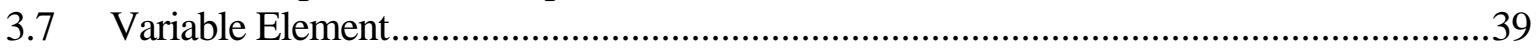

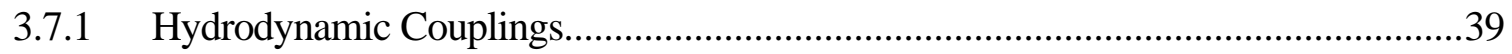

3.7.2 Hydrostatic Drives.........................................................................................41 
Chapter 4 Conceptual Design Procedure

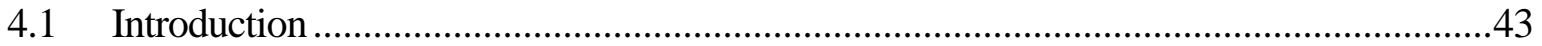

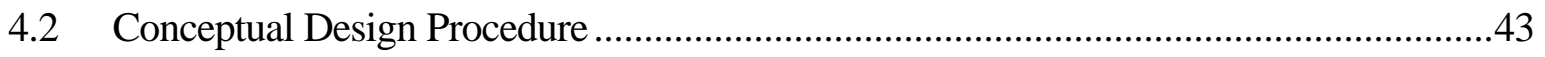

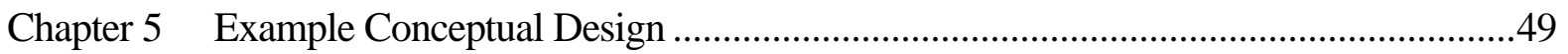

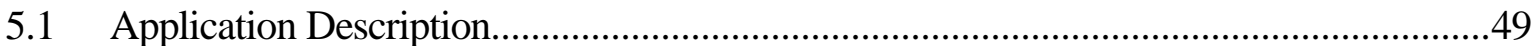

5.2 Conceptual design using a hydraulic coupling .....................................................50

5.3 Conceptual design using a hydrostatic drive .....................................................61

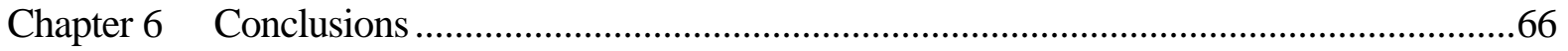

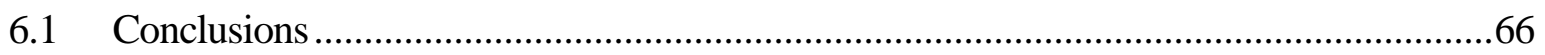

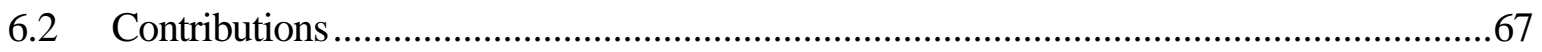

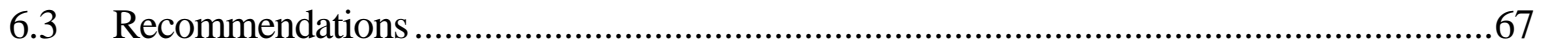

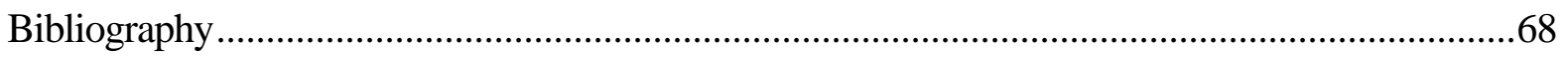

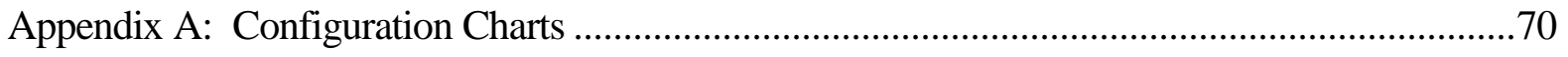

Appendix B: Gear Sizes of Example Hydrodynamic Coupling Conceptual Design....................86

Appendix C: Gear Sizes of Example Hydrodynamic Hydrostatic Drive Conceptual Design ........89

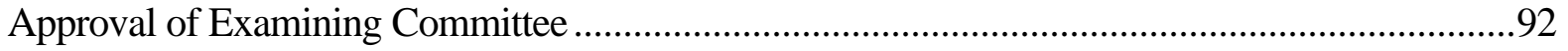


Table 1.1: Classification of shaft to shaft continuously variable transmissions ............................. 2

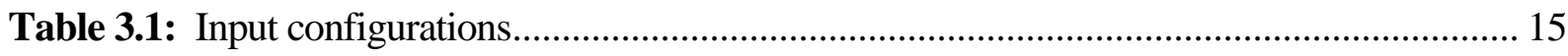

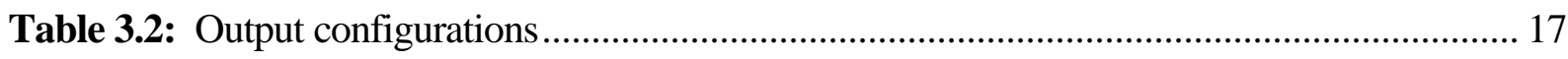

Table 5.1: Dimension comparison of developed PS-CVT concept with automatic transmissions.59 


\section{List of Figures}

Figure 3.1: The three basic variable element and planetary gear arrangements ............................13

Figure 3.2: Example configurations of split power transmission. ………………………............ 14

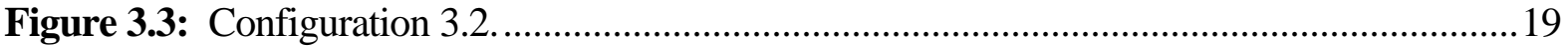

Figure 3.4: Free body diagram of configuration 3.2............................................................21

Figure 3.5: Velocity ratio curve for configuration 3.2 with arbitrary values of $\gamma_{\mathrm{h}}, \gamma_{\mathrm{g}}$, and $\gamma_{\mathrm{c}} \ldots \ldots . .24$

Figure 3.6: Ideal power flow through configuration 3.2.........................................................2

Figure 3.7: Gamma vs. $\gamma_{\mathrm{h}}$ for configuration 3.2 using arbitrary values ........................................30

Figure 3.8: Power balance block diagram ...........................................................................

Figure 3.9: Example power balance curves for configuration 3.2..............................................33

Figure 3.10: Estimated overall efficiency for example configuration 3.2 ……………...............34

Figure 3.11: Possible combinations of $\gamma_{\mathrm{g}}$ and $\gamma_{\mathrm{c}}$ for configuration 3.2 with a span of 1.5 .............35

Figure 3.12: Gamma vs. $\gamma_{\mathrm{g}}$ for configuration 3.2 with various spans ............................................37

Figure 3.13: Absolute gamma vs. the ratio coverage of the variable element for configuration 3.2 ..................................................................................

Figure 3.14: Comparison of the three combinations of $\gamma_{\mathrm{g}}$ and $\gamma_{\mathrm{c}}$ from Figure $3.10 \ldots \ldots \ldots \ldots \ldots . . . . . . . . .38$

Figure 3.15: Voith SVN hydraulic variable speed turbo coupling. ..................................................39

Figure 3.16: Relative torque versus speed ratio $\left(\omega_{\text {out }} / \omega_{\mathrm{in}}\right)$ for hydrodynamic coupling from the Voith Catalog. ................................................................................................. 40

Figure 3.17: Hydrostatic transmission configuration with a variable pump and motor.................41

Figure 3.18: Overall efficiency at maximum displacement for Sauer-Sundstrand Series

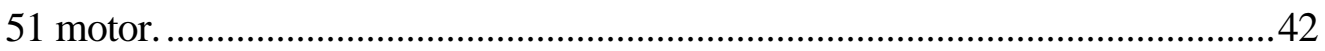

Figure 4.1: Conceptual design procedure flow diagram............................................................4

Figure 5.1: Block diagram of automatic transmission to be replaced in example application......50

Figure 5.2: Possible combinations of $\gamma_{\mathrm{g}}$ and $\gamma_{\mathrm{c}}$ which yield a span of 1.52 for example

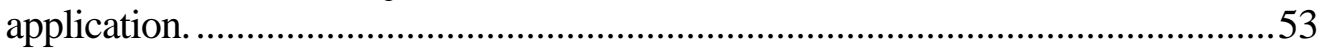

Figure 5.3: Power balance curves for example application........................................................55

Figure 5.4: Block diagram of PS-CVT design using a Voith coupling for a well service pump.

Figure 5.5: Block diagram of PS-CVT design using a Voith coupling for a well service pump..58

Figure 5.6: $2 \mathrm{D}$ rendering of the conceptual design using a coupling 
Figure 5.7: Estimated efficiency of the conceptual design using coupling...............................61

Figure 5.8: Description of example conceptual design system with hydrostatic drive ................62

Figure 5.9: Block diagram of PS-CVT design using a hydrostatic drive for a well service

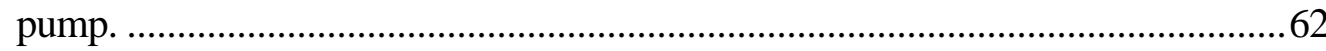

Figure 5.10: Power balance curve for conceptual PS-CVT using a hydrostatic drive.................63

Figure 5.11: Estimated efficiency of the PS-CVT with hydrostatic drive. ................................63

Figure 5.12: 2D illustration of the PS-CVT utilizing a hydrostatic drive ..................................65 


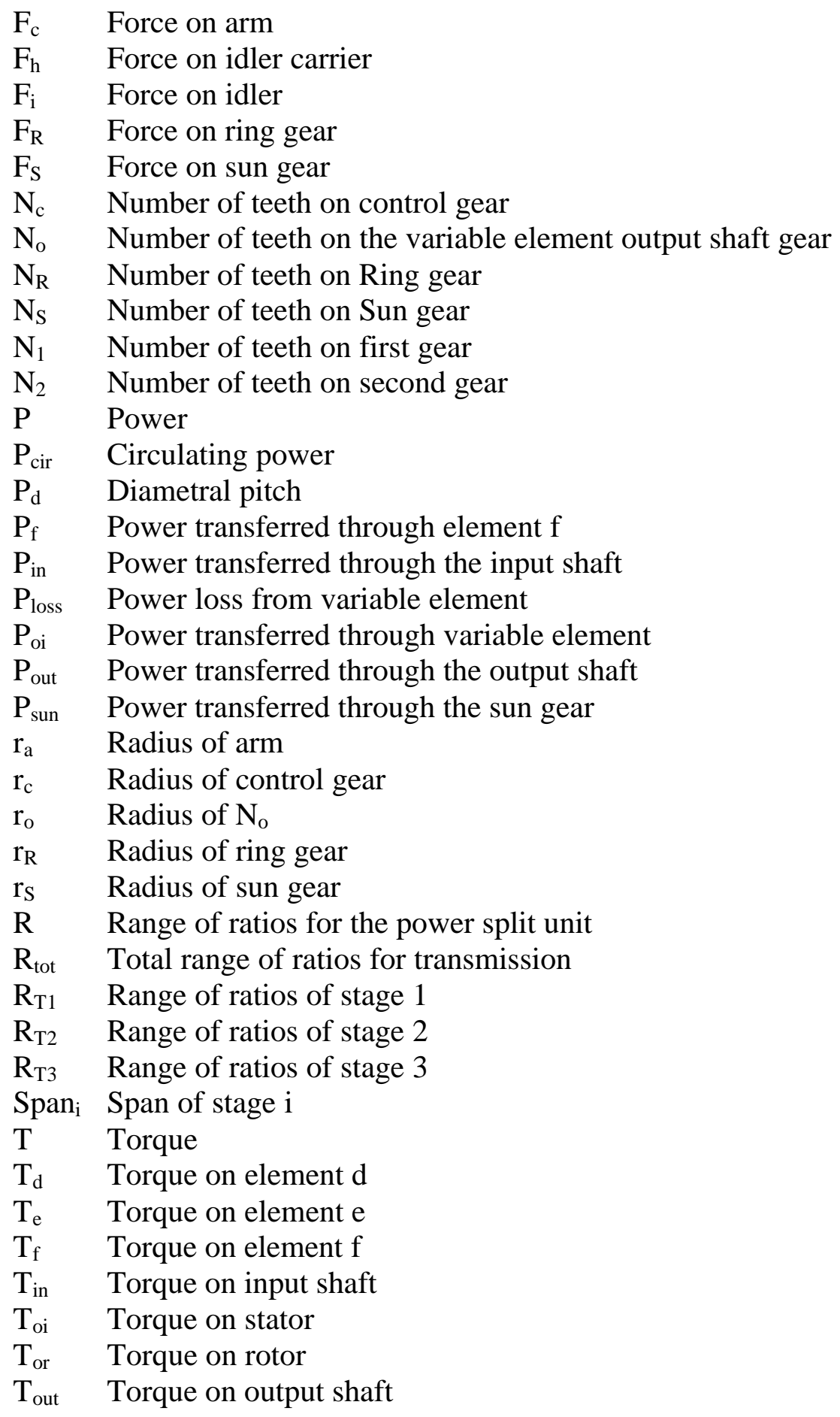




\title{
Greek Letters
}

\author{
$\gamma_{\mathrm{c}} \quad \mathrm{N}_{\mathrm{c}} / \mathrm{N}_{\mathrm{o}}$ \\ $\gamma_{\mathrm{g}} \quad \mathrm{N}_{\mathrm{R}} / \mathrm{N}_{\mathrm{S}}$ \\ $\gamma_{\mathrm{h}} \quad$ Angular velocity ratio of variable element \\ $\gamma_{\mathrm{hH}} \quad$ Highest angular velocity ratio of variable element \\ $\gamma_{\mathrm{hL}} \quad$ Lowest angular velocity ratio of variable element \\ $\gamma_{\mathrm{n}} \quad \mathrm{N}_{1} / \mathrm{N}_{2}$ \\ $\gamma_{\mathrm{w}} \quad$ Worm gear ratio \\ $\gamma_{1} \quad$ Gearbox ratio for stage 1 \\ $\gamma_{2}$ Gearbox ratio for stage 2 \\ $\gamma_{3} \quad$ Gearbox ratio for stage 3 \\ $\Gamma \quad$ Fraction of power which flows through variable element \\ $\eta \quad$ Variable element efficiency \\ $\omega_{c} \quad$ Angular velocity of control gear \\ $\omega_{\mathrm{d}} \quad$ Angular velocity of element d \\ $\omega_{\text {driven }}$ Angular velocity of the driven gear \\ $\omega_{\text {driver }}$ Angular velocity of the driver gear \\ $\omega_{\mathrm{e}} \quad$ Angular velocity of element $\mathrm{e}$ \\ $\omega_{\mathrm{f}} \quad$ Angular velocity of element $\mathrm{f}$ \\ $\omega_{\text {in }} \quad$ Input angular velocity \\ $\omega_{0} \quad$ Angular velocity of $\mathrm{N}_{\mathrm{o}}$ \\ $\omega_{\text {out }}$ Output angular velocity \\ $\omega_{\mathrm{p}}, \omega_{\mathrm{m}}$ Angular velocity of the pump \\ $\omega_{\mathrm{A}} \quad$ Angular velocity of arm relative to fixed link \\ $\omega_{\mathrm{F}} \quad$ Angular velocity of the first gear in train relative to fixed link \\ $\omega_{\mathrm{FA}} \quad$ Angular velocity of the first gear in train relative to arm \\ $\omega_{\mathrm{L}} \quad$ Angular velocity of the last gear in train relative to fixed link \\ $\omega_{\text {LA }}$ Angular velocity of the last gear in train relative to arm \\ $\omega_{\mathrm{R}} \quad$ Angular velocity of ring gear \\ $\omega_{S} \quad$ Angular velocity of sun gear \\ $\omega_{\mathrm{T}} \quad$ Angular velocity of the output of power split unit
}




\section{Chapter 1 Introduction}

\subsection{Introductory Remarks}

Transmissions are used to transmit torque and rotary motion from a power source to an output device. Two main types of transmissions are generally used for automotive and industrial applications. These are broadly classified as, geared and continuously variable. Geared transmissions consist of several fixed gear ratios and use discrete shifts to switch between them. With this type of transmission, the input/output angular velocity ratio is fixed for each gearshift. Therefore, to change the output velocity, the speed of the input device (power source) must be varied. On the other hand, continuously variable transmissions (CVTs) cover a continuous range of ratios, allowing the output speed to be varied while the input speed remains constant. Continuous ratios can offer an advantage over discrete shifts in some industrial applications. An example, is in high pressure pumping operations. In this application, the power lapses that occur during discrete shifts introduce the possibility of high pressures stalling the pump. This can result in severe damage to the pumping unit and power source.

For industrial applications where weight is a major concern, a power split continuously variable transmission (PS-CVT) would have advantages over shaft to shaft applications of a hydraulic CVT. Hydraulic CVTs that can handle high power requirements are very heavy. By reducing the power that flows though the hydraulic element, the overall size and weight can be reduced.

In the design exercise, a transmission designer is given a specific power source and an output device, and is given the task of developing a PS-CVT to suit the industrial application. 
In the design process, many obstacles need to be overcome. These include selecting the appropriate configuration of planetary gear trains and continuously variable element. Development of a design procedure will allow the designer to determine the feasibility of a PS-CVT concept for a specific application.

\subsection{Continuously Variable Transmissions}

Continuously variable transmissions can be classified into two major groups; shaft to shaft drives and power split (PS-CVT) continuously variable transmissions. Shaft to shaft CVTs are transmissions in which $100 \%$ of the power being transmitted flows through the variable element. These can be sub-classified into the five groups listed in Table 1.1, below (Lu 1998, Kluger and Fussner 1997). Several applications have used these types of transmissions.

\begin{tabular}{|c|c|}
\hline CVT Types & Examples \\
\hline All Gear & Epicyclic \\
\hline Traction & Toroidal and Nutating \\
\hline Hydraulic & $\begin{array}{c}\text { Hydrostatic, Torque converter, } \\
\text { and Coupling }\end{array}$ \\
\hline Chain & Chain \\
\hline Belt & $\begin{array}{c}\text { Flat, Rubber V, and Metal } \\
\text { Pushing }\end{array}$ \\
\hline
\end{tabular}

Table 1.1: Classification of shaft to shaft continuously variable transmissions.

\subsubsection{Shaft to shaft CVTs}

Shaft to shaft continuously variable transmissions have also been used in a wide range of applications ranging from automotive and utility vehicles (with relatively low power requirements) to large power plants. CVTs with rubber V belts have been used successfully in utility vehicles such as golf carts, snowmobiles, and SAE Mini-Baja cars. Metal pushing 
belts, toroidal, and nutating transmissions have been used in automobiles. These CVTs, however, have not yet been widely accepted although development for automotive applications continues. Hydrostatic drives have been used on construction equipment and farm equipment such as: cranes, dump trucks, front end loaders, and tractors. However, hydrostatic drives are generally heavy and are used where weight is not a concern and high

power needs to be transmitted at relatively low speeds. Hydrodynamic couplings and torque converters have been placed in high power applications such as power plant generators and work best at high speed ranges.

\subsubsection{Power Split CVTs}

Power split transmissions use differentials in combination with a variable element, such as a CVT. As the name implies the power is split so that a fraction of the power circulates through the CVT element to the differential while the remaining power circulates directly thought the differential. This type of CVT is a compromise between the ratio coverage of the variable element and the amount of power that is transferred through the variable element. Power split transmissions have the advantage of transferring powers that are greater than the capacity of the variable element, which is the limiting factor in most CVT applications.

To overcome the limitations of the narrower ratio coverage, the concept of a stepless multistage PS-CVT has been developed in this thesis. This type of transmission uses an external gearbox to amplify the ratios that the PS-CVT covers. Though this type of transmission incorporates shifts, these are considered stepless since the transmission ratio remains the same before and after the shift and the impact on engine speed is minimal. When the gearshifts occur the engine experiences only a slight decrease in velocity due to inertial 
loss of the driven device during the power lapse. The engine, however, does not have to reduce its velocity through its complete operating range, as it would with a discrete gear shift transmission.

As with shaft to shaft continuously variable transmissions, PS-CVTs have been developed for several applications. Conceptual designs have been developed for motorcycles. Single and multistage PS-CVTs have been developed for automotive applications using metal push belts and hydrostatic drives for the variable element. Multistage PS-CVTs have been developed for farm tractors using hydrostatic drives. Finally, hydrodynamic couplings and torque converters have been used to develop PS-CVTs for power plants, steel mills, and offshore and onshore pipeline systems.

\subsection{Objectives}

The main objective of this study is to develop a conceptual design procedure for a power split continuously variable transmission for an industrial application using a hydraulic variable element. The objective is to be accomplished through the completion of the following tasks:

1. A literature review on continuously variable transmissions with emphasis on power split multistage continuously variable transmissions.

2. Development of a design procedure for a conceptual design of a multistage power split continuously variable transmission.

3. Development of an example of an industrial application, to illustrate the design procedure. 


\section{Chapter 2 Review of Relevant Literature}

\subsection{Power Split Continuously Variable Transmission Configurations}

A search for relevant literature pertaining to power split continuously variable transmissions revealed that substantial work has been done in this area of transmission design. Several combinations of components and differentials have been used to develop power split continuously variable drives. Noted examples are illustrated below.

Lemmens (1972) described a power split continuously variable transmission that consists of a V-belt drive and a differential. The input shaft delivers the input power directly from the driving device to the driving gear of a chain drive and the driving pulley of the Vbelt drive. The chain drive connects the input shaft to the planetary carrier of the differential. The driven pulley of the V-belt drive is connected to the sun gear. The output shaft of the transmission is connected to the ring gear. The chain drive carries the larger percentage of input power to the differential, while the lower percentage is transferred through the V-belt. Infinitely variable speed ranges in both forward and reverse directions, and a geared neutral condition can be achieved by varying the $\mathrm{V}$-belt drive ratio.

Kumm (1991) presented a regenerative transmission that uses a flat belt CVT and a normal planetary mechanism. This transmission increased the efficiency and capacities of a flat-belt CVT by reducing the power which flows through it and permits the use of a CVT over a full speed range with two modes of operation. A high and low speed mode. In low speed mode, the input power is divided into two paths. The first path is through the planetary gearing to the output while the second path is from the planetary gearing through the CVT back to the input shaft. In high speed mode, the clutch that connects the output shaft to the 
ring gear of the planetary gear train is disengaged. Then, another clutch is engaged which permits the input power to be transmitted directly through the CVT to the output shaft. Reverse output speeds are made available by changing the velocity ratio control of the CVT in the low speed range without actuating clutches. This regenerative PS-CVT arrangement eliminates the capacity limits of the flat belt, but the power transmitted by the gear train is larger then the input power.

Takayama et al. (1991) combined a V-belt and a two way clutch to create a PS-CVT. Once the power from the prime mover is transferred to the input shaft of the transmission, two paths are able to transmit the power from the shaft. The main transmitting path is through the V-belt drive. The sub-power transmitting path consist of a two way clutch. When the V-belt is near the maximum drive ratio, the rotational speed of the output gear of the two way clutch becomes slower then its input gear. This results in power transfer from both the main path and sub-path to the output shaft of the transmission. This makes it possible to increase driving power and torque and also to decrease the load carried by the Vbelt. The benefit of this transmission over previous PS-CVTs is that the overall power capacity is increased while the weight is decreased due to a reduced number of clutches. This is a less optimum PS-CVT, because most of power is transmitted through the V-belt.

Cowen $(1992,1993)$ presented a PS-CVT design that combines a variable speed transmission unit connected with a planetary gear train. Cowen's design allowed the use of any type of variable speed unit. In this configuration the input was connected to the input of the variable speed unit and the sun gear of the planetary gear train. The output of the variable speed unit was connected to a secondary shaft. This shaft is connected to the ring gear of the planetary gear train by a control gear or gears. The output shaft is connected to 
the planetary carrier. When the secondary shaft and the ring gear are connected such that they rotate in the same direction, the input power is divided into two paths. The first is directly through the sun gear to the output shaft. The second is through the variable speed unit, secondary shaft, control gears, and the planetary gear train to the output shaft. With this transmission configuration, it is possible to achieve a function such that only a fraction of the total power load is transmitted through the variable speed transmission unit.

A family of configurations based on a simple planetary-CVT presented by Cowen (1992, 1993) was presented by Mucino et al. (1995). This set of eight PS-CVT configurations contained two planetary gear trains and a CVT unit. The combination of these three main sub-systems does not require the use of clutches or chain drives. The corresponding equations for the transmission velocity ratio and belt forces were derived for each configuration. These systems can be adjusted with relative ease to match the input/output requirements for various applications. A particular feature that this system offers is the possibility of designing the mechanism in such a way that the power circulation is directed to take place through the gear sets at low speeds, just when the requirements for torque are greatest.

\subsection{Extended range PS-CVTs}

A power split continuously variable transmission represents a compromise design since the variable speed range of the transmission is narrowed to reduce the amount of power that flows through the variable element. Much research has been devoted to extending the range of PS-CVTs. The various methods that have been used are discussed below. 
Orshansky and Weseloh (1972) investigated the characteristics of a multiple range hydromechanical transmission. The transmission consisted of a variable displacement pump, fixed displacement motor, two planetary gear trains, and several clutches. The transmission presented had two hydrostatic modes and three power split modes. In hydrostatic modes, the input power was transmitted through the hydrostatic drive. The output was coupled to one of the two planetary gear trains by clutching. Two power split modes were accomplished by clutching the input shaft to a shaft that contained the sun gears of both planetary gear trains. As in the hydrostatic modes the output was connected to one of the planetary gear trains by clutching. A third power split mode occurred when the second planetary gear train was connected to the output element, and the hydrostatic drive was taken back and used through its full range. It was concluded that multirange transmissions have the advantage of high efficiency and the ability to transmit large horsepower with hydraulic units of relatively small size. They are also increasingly advantageous where conditions require wider torque variation at full power and a high efficiency over a wider range then single stage PS-CVTs.

White (1974) derived two families of variable-ratio transmission mechanisms. Each of these mechanisms employ two coaxial differential gears and an input coupled variable ratio unit. The two families of mechanisms are classified as two path twin differential and two path cross coupled differential gear trains. The transmissions provide variable speedratios from a minimum number of geared units and allow transfer between two drive-ranges by means of clutches engaged at zero speed-difference. A method was presented to determine the speed ratios for each stage. Since the shift between the two stages occurs at a zero speed difference, the feasibility of each arrangement could be determined. A table was developed 
which ensures an arrangement can be chosen from these two families with confidence that all solutions have been covered.

Hausenbauer (1976) presented computer models that investigated the effects of adding a PS-CVT in series to a 4-speed manual gearbox in automobiles. The power split transmission was of the input coupled type utilizing a hydrostatic drive as the variable element. Stepless shifts of the manual gearbox extended the range of the PS-CVT. The computer simulation work resulted in projected fuel economy improvements of approximately $50 \%$ over that of the conventional 4-speed gearbox. The model was also used to assess the ease with which the vehicle could be driven.

White (1977) illustrated another configuration in which a gearbox was used to extend the range of a PS-CVT. The two-speed gearbox connects to the variable element output shaft to the differential. The shift between the two gear ranges occurs when the velocity of the hydrostatic drive is zero. Therefore, there is no discontinuity in the transmitted torque and there is no wear on the clutch. This unit required further gear trains to allow zero and reverse output speeds to be achieved through synchronous shift clutches. This imposes a penalty in the form of a decreased power/weight ratio. The mechanism represents one of a number of possible approaches to the problem of improving the performance of stepless variable transmissions while widening the spread of speed ratios.

\subsection{PS-CVT Design for Applications}

Power split continuously variable transmissions have been designed for several applications. Most of the literature found was for vehicles including automobiles, buses, and 
motorcycles. Several methods of designing and analyzing PS-CVTs for specific applications are presented below.

Beachley et al. (1984) evaluated a power split continuously variable transmission for automotive applications. The study focused on PS-CVT systems using a single differential with parameters such that the ratio coverage is extended beyond that of the continuously variable unit. Both single regime and two regime systems were considered. It was not necessary to consider the mechanical details of either the variable unit or the differential because the work was based on mathematical relationships. The data developed in this study can serve as a guide to help determine whether transmissions of this type would be suitable, from efficiency considerations for use in conventional automobiles. The results of this study suggest that no split path extended range CVT should be considered for automotive applications without first performing a realistic analysis of the efficiency aspects. The analysis should be based on the efficiency characteristics of the particular continuously variable unit to be used, as the efficiency penalty can be high in many cases.

Dorey and Vaughan (1984) investigated the design of the power split transmission for a vehicle drive and illustrated how the transmission characteristics could be matched to the particular requirements of the vehicle duty. This design study was accomplished using computer aided design due to the number of design alternatives available. A unified approach to the modeling procedure was used to enable computer programs, which describe the behavior of the complex transmissions, to be easily constructed and altered. This study stressed the importance of computer models in the design of PS-CVTs since they reduce the time required to develop a new transmission and greatly facilitate evaluation of modifications to the transmission configuration. 
Sheu (1996) presented a procedure for the conceptual design of hybrid transmissions for motorcycle applications. The hybrid transmissions are developed using combinations of fixed gear ratio, split power, and continuously variable transmission systems. Four design concepts were synthesized. These included: split power, split power and continuously variable, fixed gear and continuously variable, and fixed gear and split power transmission systems. Using a V-belt drive continuously variable unit and simple differential gears, topological structures of the hybrid transmissions were created. The feasibility of the proposed procedure was illustrated with a design example.

Lu et al. (1999) presented a simulator program that aids in the design and development of a power split continuously variable transmission. The simulator can be used to optimize the transmission and compare it with other transmissions. The objective of the design simulator was to develop a PS-CVT design to minimize the loading on the belt while providing for increased power transfer compared to existing designs. An optimized design was presented. A vehicle acceleration simulation indicated that the optimized PS-CVT could provide a continuously variable ratio change. It also illustrated that the fuel economy of a vehicle with a PS-CVT is better than that of a standard or a multi-ratio automatic transmission.

Although the literature review revealed several designs for specific applications, these configurations are typically for low power systems. For larger power industrial applications, PS-CVT units are not well documented. Units that fulfill these larger power requirements are address in this thesis. 


\section{Chapter 3 PS-CVT Concepts and Components}

\subsection{Overview}

Before the process of designing a split-power drive for heavy duty applications can be described, the main components that comprise the design are explained. These components include the input and output gear arrangements, the velocity ratio, power flow through the variable element, and the variable element itself. For each of these components, the kinematic relationships and performance curves are presented for the overall design of a power split transmission. These components are then used to describe the design process with an example application.

\subsection{Input and Output Configurations Gear Arrangements}

Variable elements and differentials can be arranged in one of three ways. The three combinations are illustrated in Figure 3.1. These three combinations can further be classified into two groups: variable shunt (Figure 3.1 A and B), and variable bridge (Figure 3.1 C). Variable shunts consist of a variable element connected to a single differential. The other end of the variable element is coupled to the input or output shaft. These configurations are referred to as input coupled variable shunt and output coupled variable shunt respectively. The variable bridge consists of a variable element bridged between two differentials. This design study focuses on the input coupled variable shunt configuration. There are two reasons for this. First, the input and output coupled variable shunts contain only one differential and are assumed lighter then the variable bridge. Second, based on the work of Hsieh and Yan (1990), input coupled variable shunt can be arranged to be mechanically more efficient than the output coupled variable shunt. 


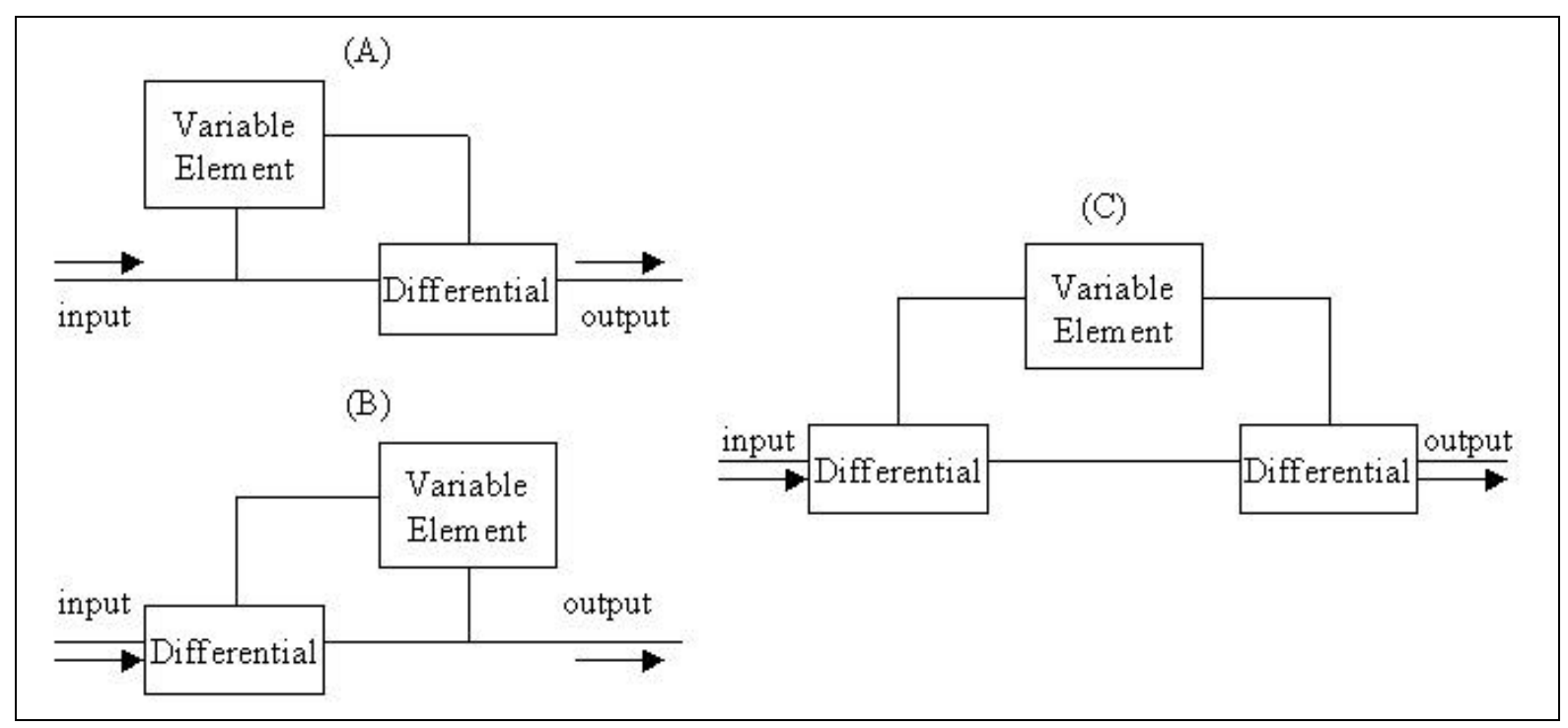

Figure 3.1: The three basic variable element and planetary gear arrangements.

A variable shunt drive consists of two main components: the branch control circuit and the differential. In all of the configurations presented, a third connecting element is used. This element connects the branch control circuit to the input shaft. It can be described as a fixed planetary, or simply a multiplier or reducer, depending on the arrangement of gears. The differential is a single planetary gear train consisting of a ring gear, sun gear, and planetary carrier in all of the configurations presented.

Two examples of typical configurations are illustrated in Figure 3.2, in which a variable transmission element is used with a through-shaft. The variable element divides the input power between the branch control unit (secondary shaft) and the primary shaft (through-shaft). Each transfers the power to the output shaft. In example 1, the input power is branched between the planet carrier and the sun gear, and the output power is transferred from the ring gear. Another possible configuration is example 2, where the output comes from the planet carrier and the input power is branched between the other two elements. These two examples also illustrate two possible multiplier/reducer elements, which connect 
the variable element to the planetary gear train. Example 1 contains a reducer, while example 2 contains a multiplier.

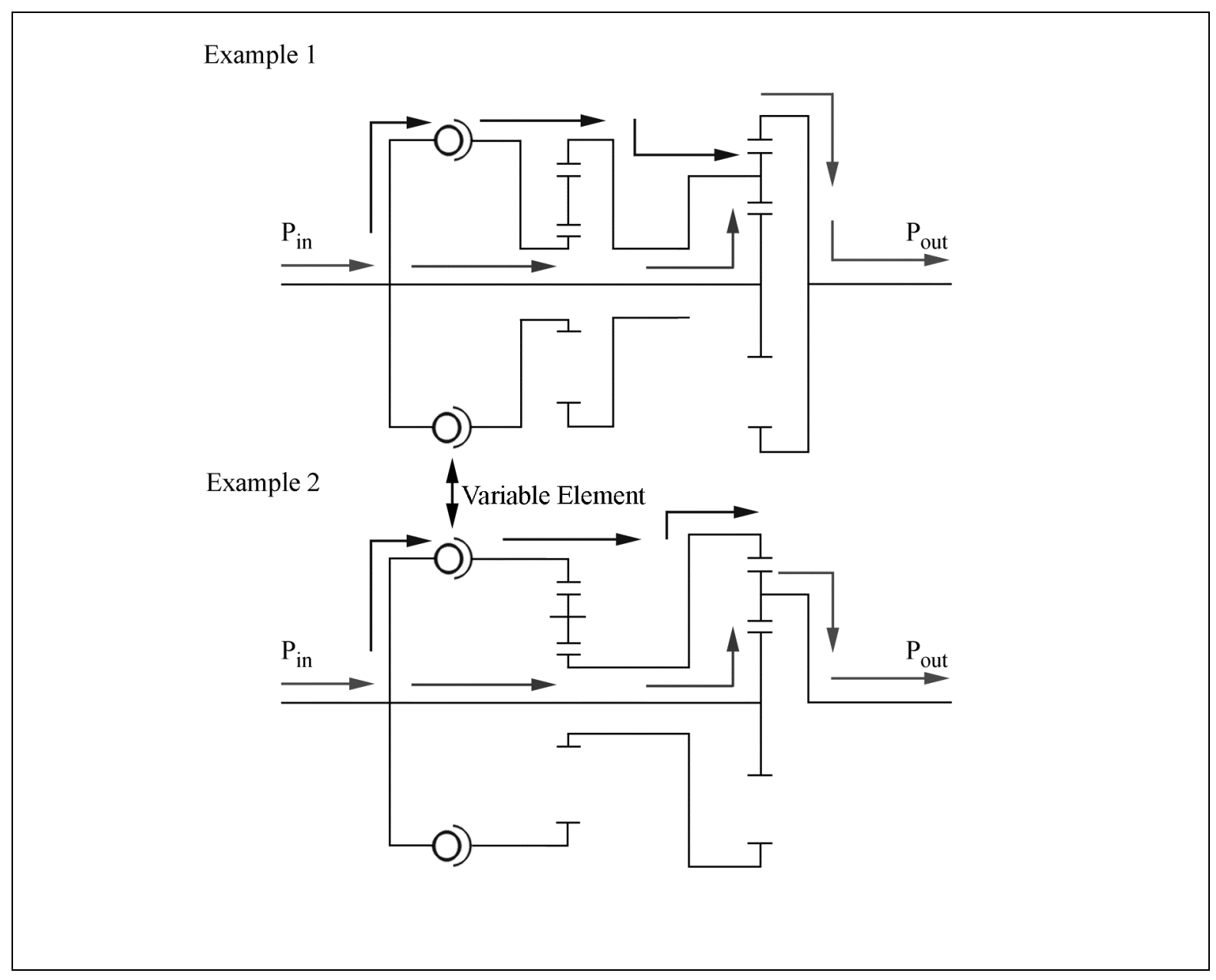

Figure 3.2: Example configurations of split power transmission.

A continuously variable transmission provides a continuous speed ratio change with a constant power flow. This is accomplished in this system by the variable element, which varies the angular velocity between the primary and the secondary shaft. The ratio of input angular velocity divided by the output angular velocity of the variable element is designated as $\gamma_{\mathrm{h}}$. By varying the angular velocity of one of the shafts, the angular velocity of one of the differential input elements is also varied. With one of the inputs of the differential constant 
and the other variable, the output angular velocity is varied. The variable element supplies a continuous flow of power through the transmission while providing a varying velocity ratio.

The configurations considered in this thesis have two main segments: one designated as input and a second one as output. The input segment contains the variable element and the multiplier/reducer, while the output segment contains the planetary gear train. Five different configurations where found for the multiplier/reducer. They can be connected in two ways to the variable element, for a total of ten possible input combinations. These combinations are illustrated in Table 3.1, which is continued on the following page. The secondary shaft can be connected to the variable element through the multiplier/reducer so that its rotation varies with $\gamma_{\mathrm{h}}$, while the primary shaft rotation is equivalent to the input angular velocity. These are configurations 1 though 5 in the table. In the other five configurations, the primary shaft's angular velocity is varied with $\gamma_{\mathrm{h}}$, and the secondary shaft rotation is equivalent to the multiplied or reduced input angular velocity.

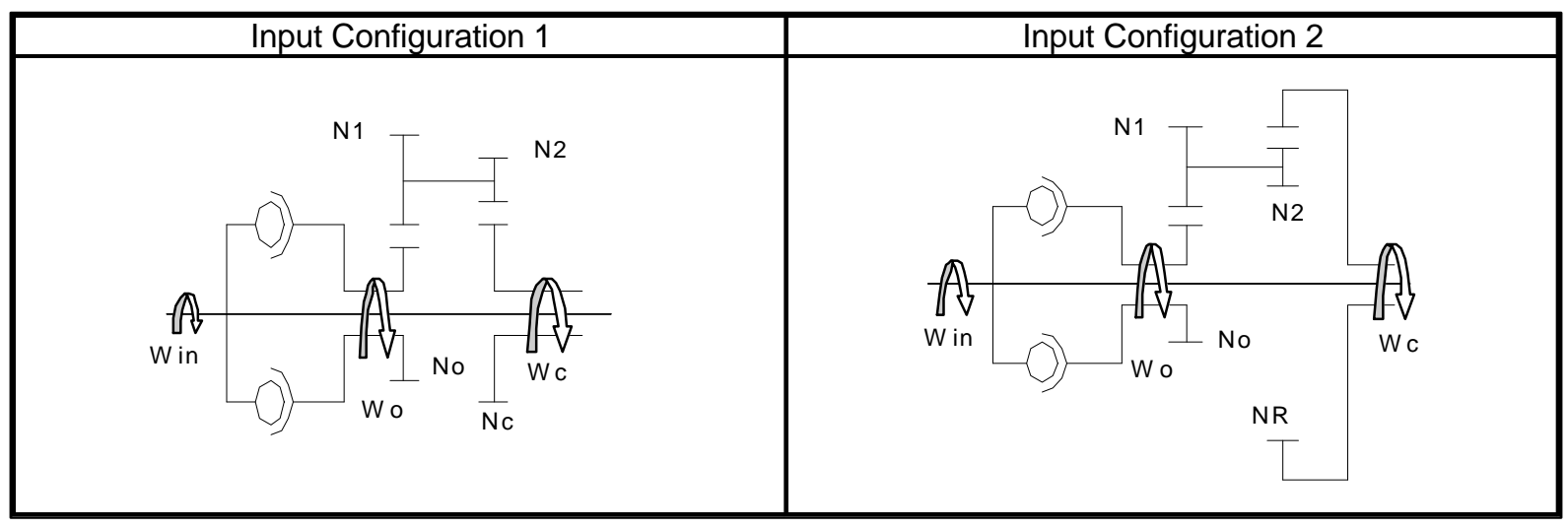

Table 3.1: Input Configurations. 


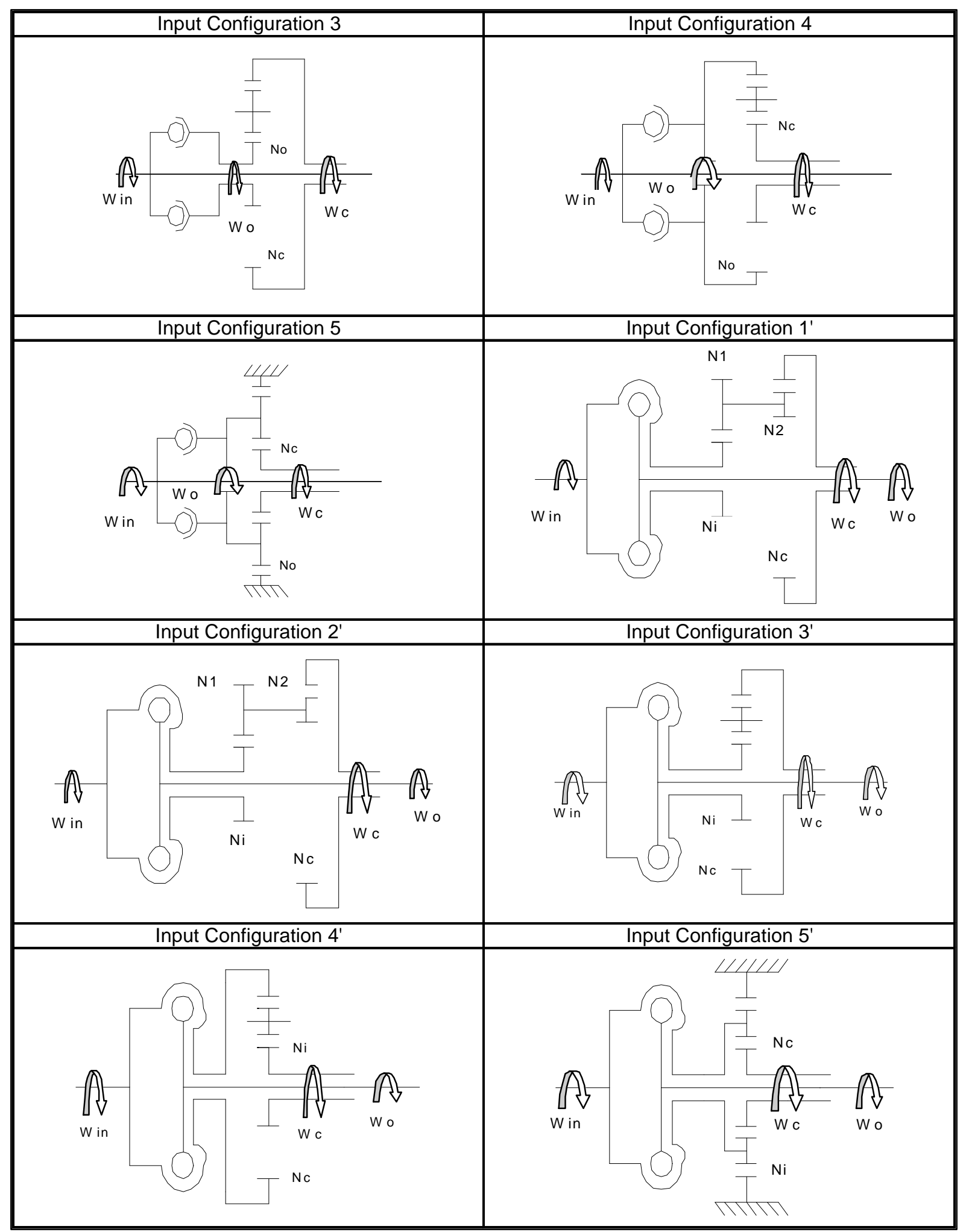

Table 3.1 continued: Input configurations. 
There are six possible combinations for two input elements and one output element of the planetary gear train. The six possible output configurations are illustrated in Table 3.2. These six, along with the ten inputs, combine for sixty different configurations. Each is designated by the name configuration, followed by the input number and then the output number. For example, the configuration that combines the input configuration 1 and output configuration 1 is designated configuration 1.1.

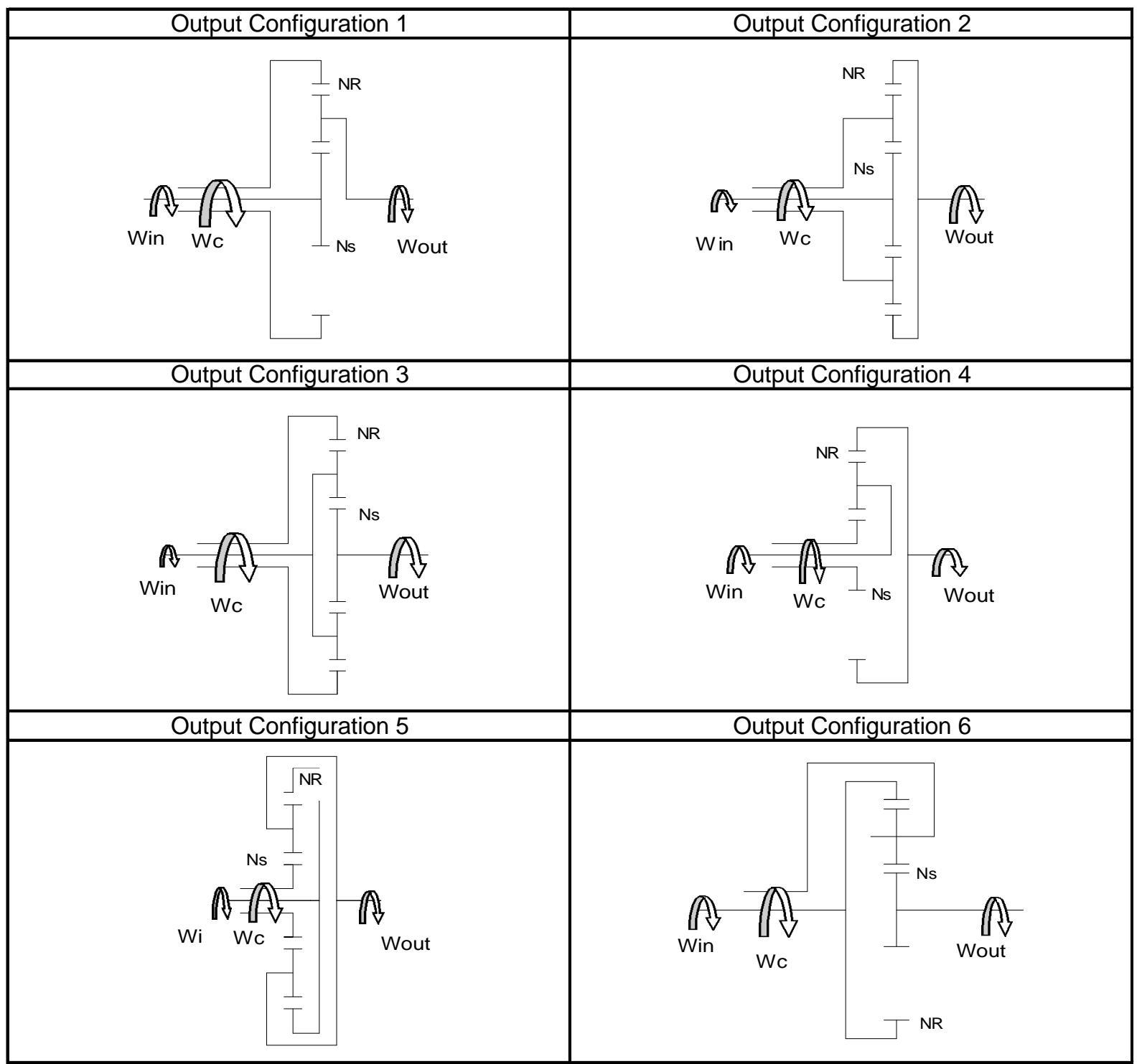

Table 3.2: Output configurations. 
To determine the best configuration for a given application, both the velocity ratio span and the fraction of power which flows through the variable element, gamma $(\Gamma)$ must be considered. The velocity ratio span, hereafter referred to simply as the span, is equal to the overall low-speed transmission ratio of the transmission divided by the overall high-speed ratio. Each configuration has its own unique span and $\Gamma$, the process for deriving these properties is discussed in the next few sections.

\subsection{Velocity Ratio}

The velocity ratio is defined as the input angular velocity divided by the output angular velocity, or $\omega_{\text {in }} / \omega_{\text {out. }}$ The variable element and the input/output configuration of the transmission each have an effect on its velocity ratio. The velocity ratio of a configuration can be derived by kinematics or force analysis. Example derivations of the velocity ratios are shown for configuration 3.2 using first kinematics, then force analysis. The two derivations are shown to provide a check. The example derivations illustrate the method used to derive the equation for the velocity ratios for all the configurations.

\subsubsection{Analysis of the Velocity Ratio using Kinematics}

In this section, kinematics is used to derive the velocity ratio, $\omega_{\mathrm{in}} / \omega_{\text {out }}$, for configuration

3.2. To derive the velocity ratio, the angular velocities of all the planetary gear train elements are used. The angular velocities of the sun and ring gears from inspection of Figure 3.3 are $\omega_{\text {in }}$ and $\omega_{\text {out }}$ respectively. The only angular velocity not known in this configuration is that of the planet carrier. To find this, an equation is derived for the input to output angular velocity ratio of the variable element. Using the notation provided by Figure 3.2 this equation is: 


$$
\frac{\omega_{i n}}{\omega_{o}}=\gamma_{h}
$$

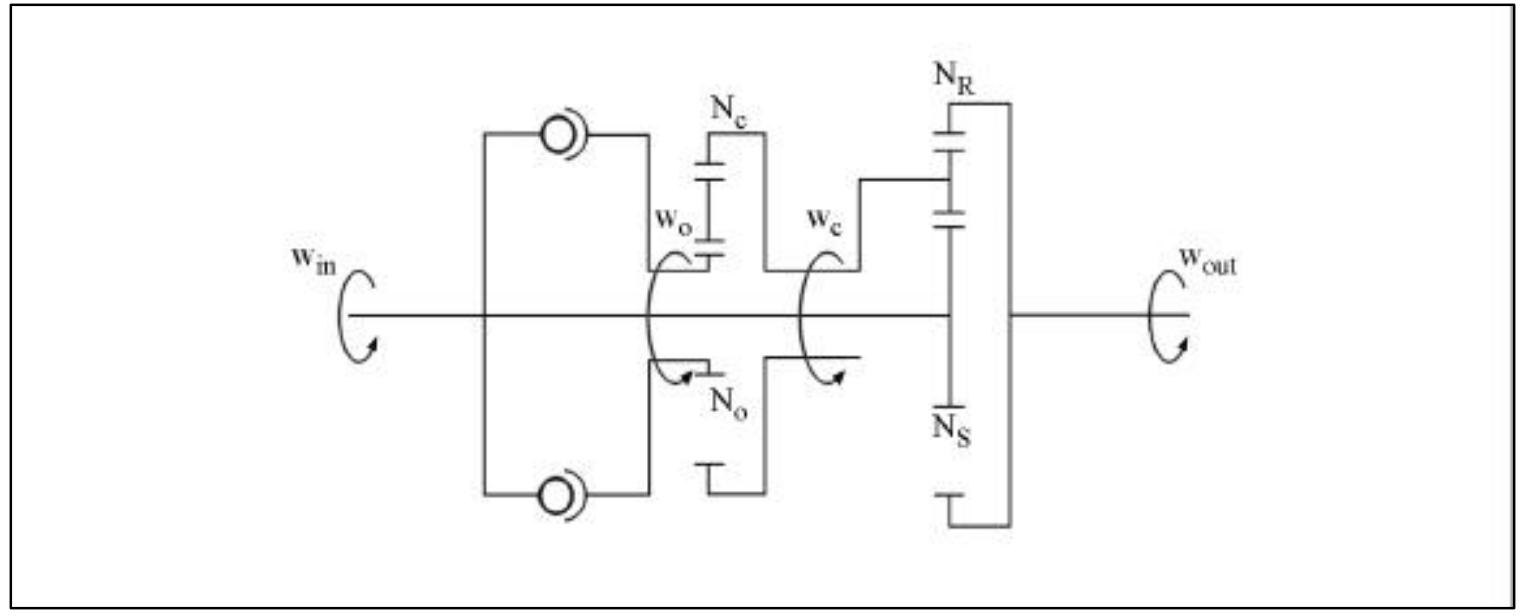

Figure 3.3: Configuration 3.2.

The relationship between $\omega_{\mathrm{o}}$ and $\omega_{\mathrm{c}}$ is then determined. This is done using the relation that $\omega_{\text {driven }} / \omega_{\text {driver }}$ is equal to the product of teeth of driving gears divided by the product of teeth of driven gears (Mabie and Reinholtz 1987).

$$
\frac{\omega_{o}}{\omega_{c}}=-\gamma_{c}
$$

Where $\gamma_{c}$ is equal to:

$$
\gamma_{c}=-\frac{N_{c}}{N_{o}}
$$

The result of solving equation 3.1 for combining with equation 3.2 may be written in terms of $\omega_{0}$ as follows:

$$
\omega_{c}=-\omega_{i n} \frac{\gamma_{c}}{\gamma_{h}}
$$


The angular velocities of all the planetary gear train elements are now known. Using the following equation for planetary gears (Mabie and Reinholtz 1987) and the previous derivation, an expression for $\omega_{\text {in }} / \omega_{\text {out }}$ can be determined.

$$
\frac{\omega_{L A}}{\omega_{F A}}=\frac{\omega_{L}-\omega_{A}}{\omega_{F}-\omega_{A}}=-\frac{N_{F}}{N_{S}}=-\gamma_{g}
$$

where

$$
\begin{aligned}
& \frac{\omega_{L A}}{\omega_{F A}}=\text { velocity ratio of last gear to first gear both relative to arm } \\
& \omega_{L}=\text { angular velocity of last gear in train relative to fixed link } \\
& \omega_{A}=\text { angular velocity of arm relative to fixed link } \\
& \omega_{F}=\text { angular velocity of first gear in train relative to fixed link }
\end{aligned}
$$

For this derivation, the ring gear is selected as the first gear in the planetary gear train and the sun gear is selected as the last gear. Substituting these in the rearranged equation 3.5 for the angular velocities of the first and last gears yields:

$$
\omega_{S}-\omega_{A}=\gamma_{g}\left(\omega_{A}-\omega_{R}\right)
$$

From inspection of Figure 3.2:

$$
\begin{aligned}
& \omega_{R}=\omega_{\text {out }} \\
& \omega_{A}=\omega_{c} \\
& \omega_{S}=\omega_{\text {in }}
\end{aligned}
$$

Substituting these and equation 3.4 for $\omega_{\mathrm{c}}$ into equation 3.6 and simplifying results in the following equation, which is the angular velocity ratio of configuration 3.2:

$$
\frac{\omega_{\text {in }}}{\omega_{\text {out }}}=-\frac{\gamma_{g} \gamma_{h}}{\gamma_{h}+\gamma_{c}\left(\gamma_{g}+1\right)}
$$




\subsubsection{Analysis of Velocity Ratio using Force Analysis}

The ratio of the input to output angular velocities can also be calculated using force analysis. The force analysis of configuration 3.2 is presented here as an alternative method to calculate the velocity ratio and as a check to the previous derivation using kinematics. Figure 3.4 is the free body diagram of configuration 3.2. This figure illustrates the forces and torques present in this configuration. By determining the relation between the input to output torque and using the assumption that there are no losses in the transmission the input to output velocity ratio is derived.

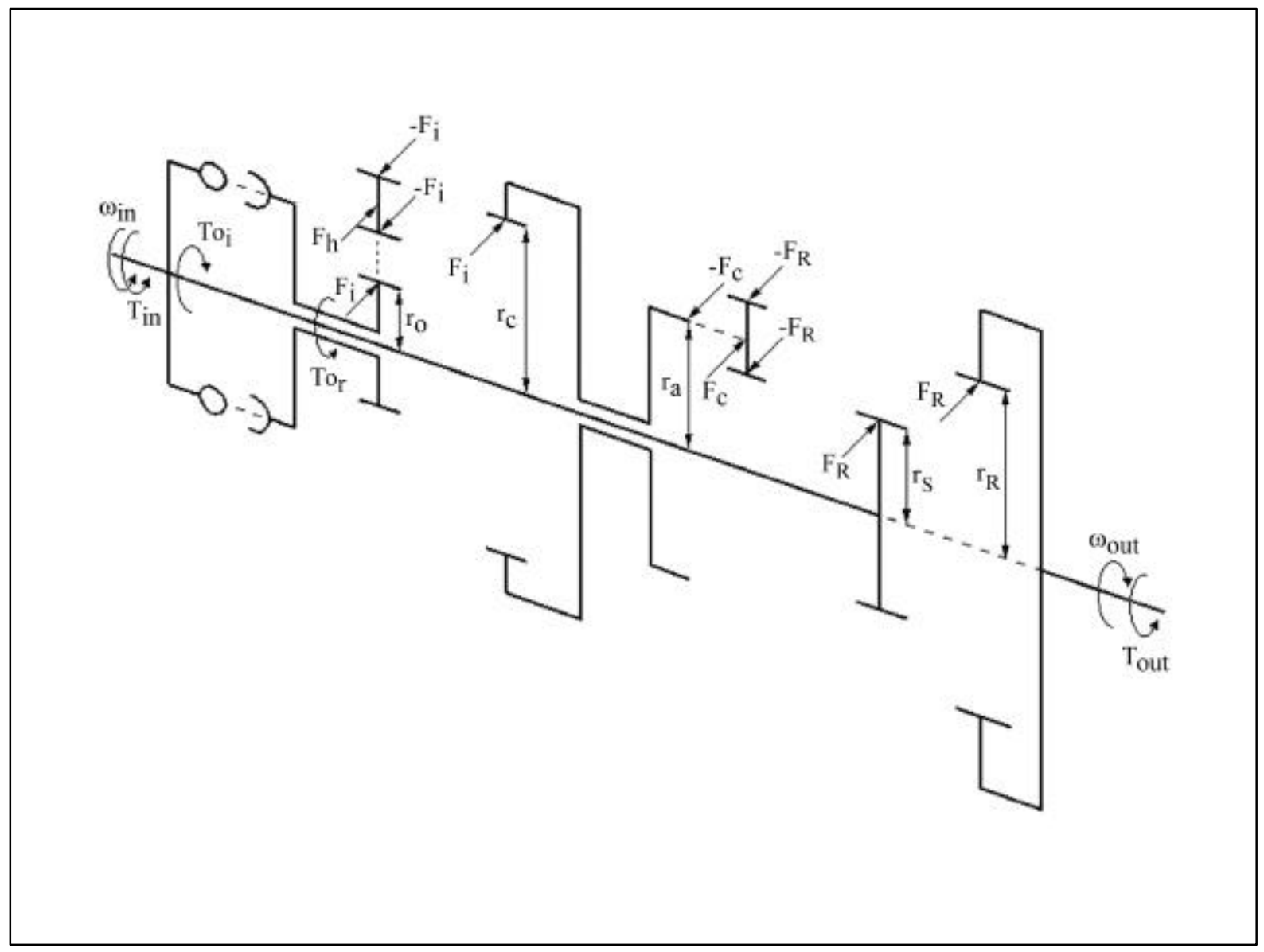

Figure 3.4: Free body diagram of configuration 3.2.

The first step in the derivation of angular velocity ratio using force analysis is to sum the torques on the input shaft. This summation yields the following equation: 


$$
\sum T=T_{i n}-T o_{i}-F_{R} r_{S}=0
$$

The torques can also be summed on the output shaft of the variable element or secondary shaft. This produces the following equation:

$$
\sum T=T o_{r}-F_{i} r_{o}=0
$$

Next, the forces on the idler gear are summed, this yields the following equality:

$$
F_{h}=2 F_{i}
$$

The torques on the planet gear carrier are summed, resulting in the following equation:

$$
\sum T=F_{c} r_{a}-F_{i} r_{c}=0
$$

Finally, the forces on the planet gear are summed, this yields:

$$
F_{c}=2 F_{R}
$$

Now, equations 3.9 through 3.11 can be substituted into equation 3.12 and simplified to produce:

$$
T o_{r}=T_{\text {out }} \frac{r_{o}}{r_{R} r_{c}}\left(r_{S}+r_{R}\right)
$$

Equation 3.8 can then be solved for $\mathrm{To}_{\mathrm{i}}$.

$$
T o_{i}=T_{\text {in }}-\frac{T_{\text {out }}}{\gamma_{g}}
$$

Assuming that the variable element has an efficiency of 1 , the power into the element equals the power out. The effect of the variable element's efficiency on the velocity ratio will be examined in a later section. The power in and power out can be expressed as a product of angular velocity and torque, which yields the following equation:

$$
\omega_{i n} T o_{i}=\omega_{o} T o_{r}
$$

Solving this for $\omega_{\mathrm{in}} / \omega_{\mathrm{o}}$ and substituting in equation 3.1 yeilds: 


$$
\frac{T o_{i}}{T o_{r}}=\frac{1}{\gamma_{h}}
$$

Equation 3.13 and 3.14 can now be substituted into Equation 3.16 and simplified to:

$$
\frac{T_{\text {in }}}{T_{\text {out }}}=\frac{\gamma_{c} \gamma_{g}}{\gamma_{h}+\gamma_{c}\left(\gamma_{g}+1\right)}
$$

Since it was assumed that the variable element has an efficiency of 1 , the total system is assumed to have the same efficiency. The total power in equals the total power out which is expressed as the following equation:

$$
\frac{\omega_{\text {in }}}{\omega_{\text {out }}}=-\frac{T_{\text {out }}}{T_{\text {in }}}
$$

Substituting equation 3.17 into equation 3.18 results in equation 3.7. The two derivations match. This method was repeated to calculate the velocity ratio for the other configurations. The results using either of the methods can be used to calculate the span of veloctiy ratios and $\Gamma$, which is covered in the following sections.

\subsection{Velocity Ratio Curve and Span of Velocity Ratios}

In the previous sections the method used to derive the velocity ratio for each configuration was presented. The equation for the velocity ratio of each configuration can be found in the configuration charts in Appendix A. In every case $\gamma_{\mathrm{h}}$ is the only variable in the equation. The other terms are based on the geometry of the configuration and therefore remain constant. Because of this, the velocity ratio can be plotted against the variator's ratio $\left(\gamma_{\mathrm{h}}\right)$, producing a velocity ratio curve. Figure 3.5 is an example curve plotted for configuration 3.2. Arbitrary values for $\gamma_{\mathrm{g}}$ and $\gamma_{\mathrm{c}}$, of 1.5 and .32 respectively, were used and $\gamma_{\mathrm{h}}$ was varied between 1 and 5. Inspection of the velocity ratio curves presented in the

configuration charts, reveals two properties of the velocity ratio. The first, is that the sign of 
the velocity ratio is either positive or negative. A positive velocity ratio signifies that the rotation of the output shaft is in the same direction as the input shaft, while a negative velocity ratio signifies the output rotates in the opposite direction as the input. The second property that is shown by the velocity curves is the relationship between the velocity ratio and $\gamma_{\mathrm{h}}$. Depending on the configuration, the velocity ratio is either proportional to or inversely proportional to $\gamma_{\mathrm{h}}$. This relationship is important in calculating the span of velocity ratios.

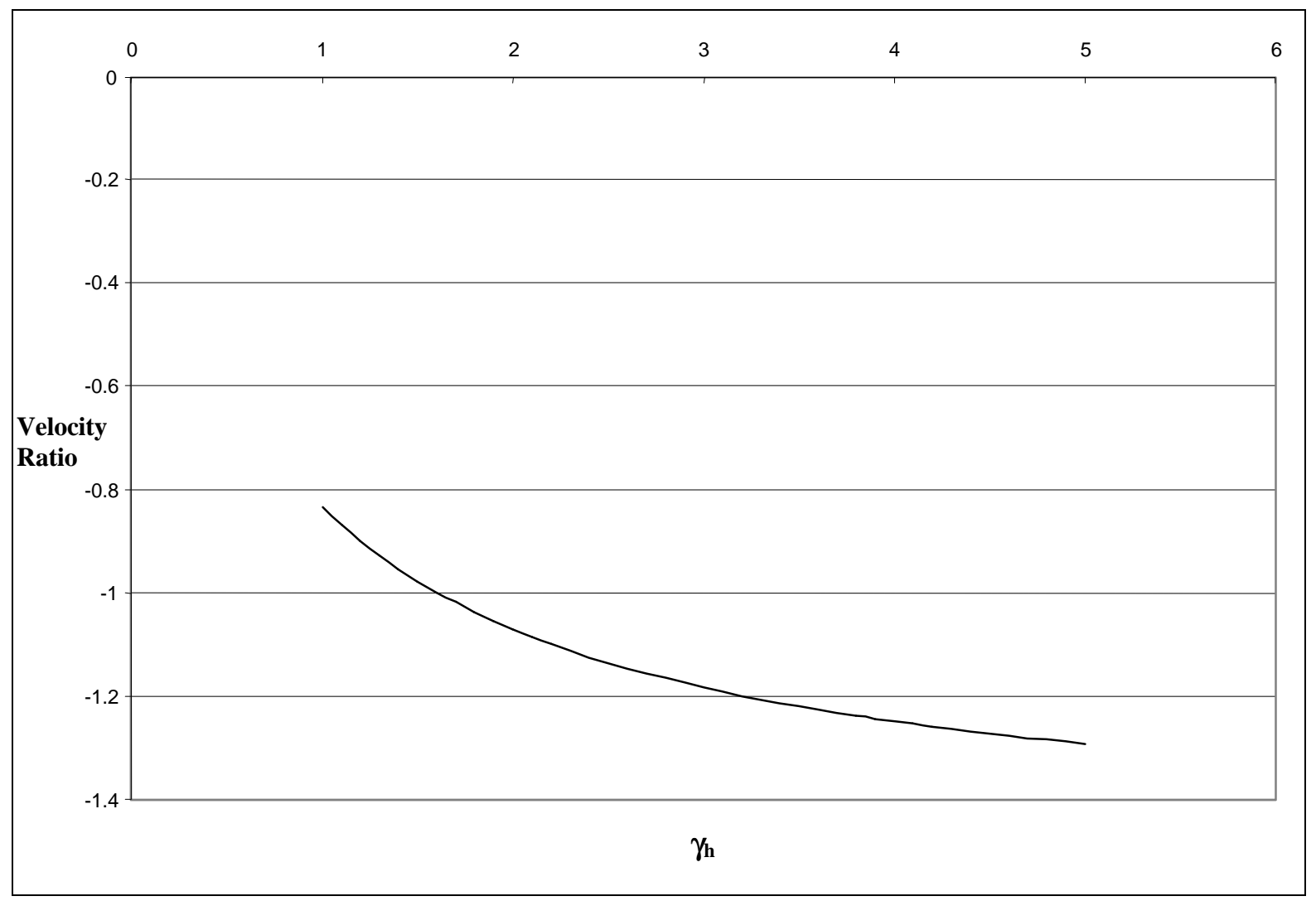

Figure 3.5: Velocity ratio curve for configuration 3.2 with arbitrary values of $\gamma_{\mathrm{h}}, \gamma_{\mathrm{g}}$, and $\gamma_{\mathrm{c}}$.

The span for a configuration is a value that describes the range of velocity ratios that is covered by that configuration. The span of a configuration is defined as its low-speed velocity ratio divided by its high-speed velocity ratio. The larger the span is for a configuration, the more velocity ratios the configuration covers. The velocity ratio curves 
can be used to compare the spans for different configurations. The larger the slope of a configuration's velocity ratio curve, the larger the span will be. The curves illustrate the trend of a configuration's velocity ratio, and allow the designer to compare the spans of different configurations without actually using specific numbers.

To calculate the span of a specific configuration, the velocity ratio equation and the relationship between the configuration's velocity ratio to the variable element's velocity ratio must be known. Equation 3.19 is the equation used to calculate the span for Configuration 3.2,

$$
\operatorname{Span}=\frac{\left[-\frac{\gamma_{g} \gamma_{h L}}{\gamma_{h L}+\gamma_{c}\left(\gamma_{g}+1\right)}\right]}{\left[-\frac{\gamma_{g} \gamma_{h H}}{\gamma_{h H}+\gamma_{c}\left(\gamma_{g}+1\right)}\right]}
$$

where $\gamma_{\mathrm{hL}}$ is the low-speed velocity ratio of the variable element and $\gamma_{\mathrm{hH}}$ is the high-speed velocity ratio of the variable element. To determine the equation of the low and high-speed velocity ratios the velocity ratio curve was used. From inspection of the velocity ratio curve of configuration 3.2 it is determined that the velocity ratio is proportional to the velocity ratio of the variable element. Therefore, the low and high-speed velocity ratios occur at the low and high-speed velocity ratios of the variable element respectively. The method to determine the equation for the span of a different configuration, consist of simply using the derived equation of the velocity ratio for that configuration and the relationship of the velocity ratio to the velocity ratio of the variable element. 


\subsection{Power Flow through Variable Element}

Gamma $(\Gamma)$ is defined as the fraction of power that circulates through the variable element. The efficiency of the variable element and the input/output configuration of the transmission each have an effect on $\Gamma . \Gamma$ can be calculated using an ideal case where the variable element's efficiency is 1 or using the actual efficiency of the variable element. Both cases are presented. The ideal case is used to determine the best configuration for a given application for two reasons. First, it is easier to derive. Second, the efficiency of the variable element will have an equal affect on all configurations. The second case, which takes into account the power loss due to the actual efficiency of the variable element, is used to specify the size of the variable element and to describe the transmission performance. The method of deriving the $\Gamma$ for each case is shown in the following two sections.

\subsubsection{Calculating Gamma in the Ideal Case}

In this section, the ideal $\Gamma$ for configuration 3.2 is derived. The example derivation describes the method used to derive the ideal $\Gamma$ for all configurations. To derive $\Gamma$, the power flow through the differential must be analyzed. The following derivation of the power flow through a differential is from Mechanisms and Dynamics of Machinery (Mabie and Reinholtz 1987).

The first step in analyzing the power flow through the differential is labeling the rotating elements of the system as elements $\mathrm{d}$, e, and $\mathrm{f}$. These are illustrated in Figure 3.6. One of these elements is the arm that caries the planetary gears and the other two are gears on independent shafts. Element $\mathrm{d}$ is always the member that projects from the differential to a 
point outside the system and connects to element e. Element e is always the member that transmits power to or from the differential to the branch control

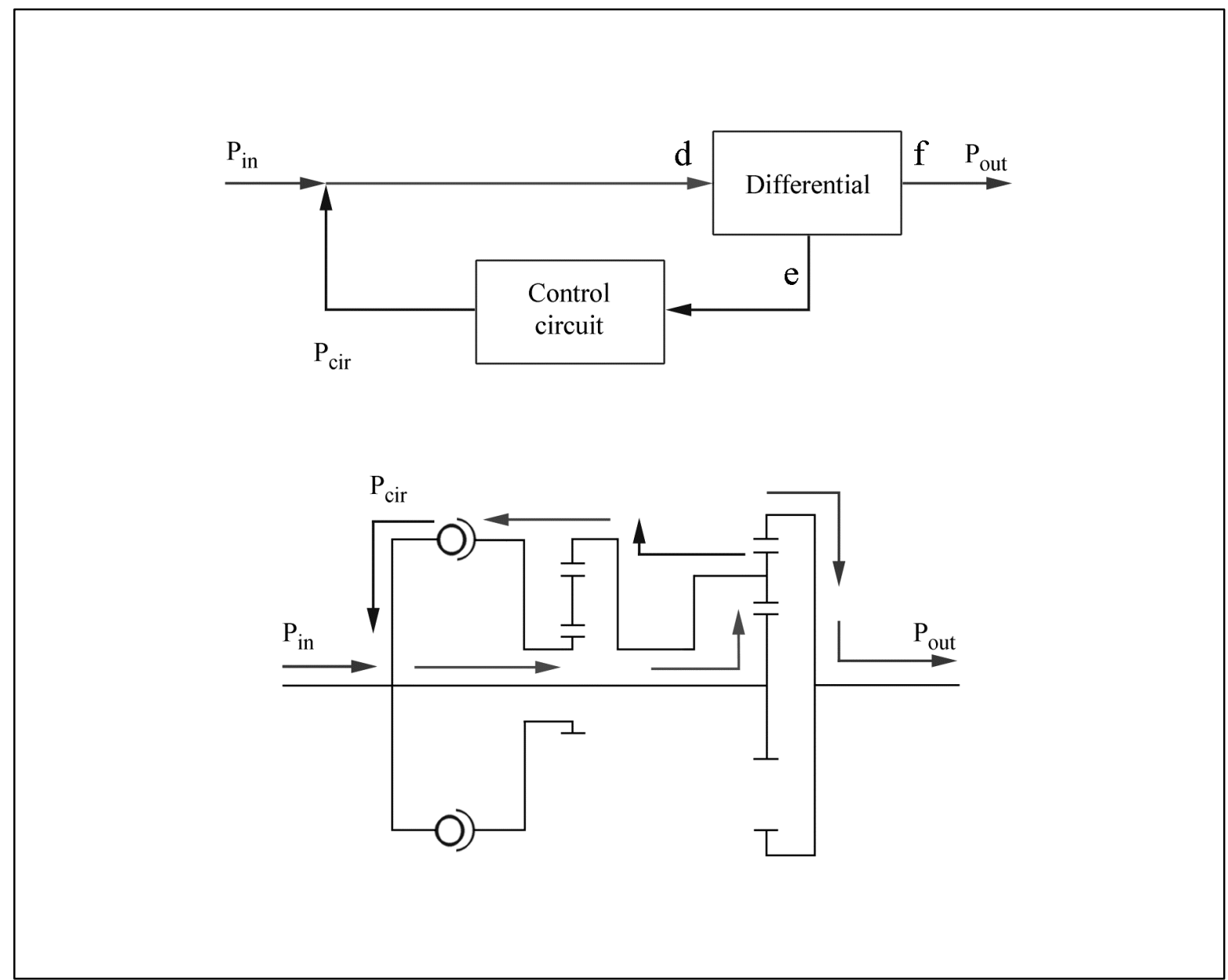

Figure 3.6: Ideal power flow through configuration 3.2.

circuit but does not transmit power directly to or from a point outside of the system. Element $\mathrm{f}$ is always the member projecting from the differential directly to a point outside the system but having no connection to the branch control circuit. Inspection of Figure 3.6 shows that for configuration 3.2 , element $\mathrm{d}$ is the sun gear connected to the input shaft, element $\mathrm{e}$ is the planetary carrier, and element $\mathrm{f}$ is the ring gear connected to the output shaft. Once the elements are defined, it can be seen that element e is the member that circulates the power 
through the variable element. Calculating the power flowing through element e gives the amount of power flowing through the variable element.

An expression for the amount of power circulating through the branch circuit can easily be developed from the general torque and energy relationships between the three elements. $\Gamma$ is defined by equation 3.20

$$
\Gamma=\frac{P_{c i r}}{P_{f}}=\frac{T_{e} \varpi_{e}}{T_{f} \bar{\varpi}_{f}}
$$

where circulating power $\left(\mathrm{P}_{\text {cir }}\right)$ is the power flowing through the branch control circuit, the power flowing through element $\mathrm{f}$ is $\mathrm{P}_{\mathrm{f}}, \mathrm{T}_{\mathrm{e}}$ and $\mathrm{T}_{\mathrm{f}}$ are the torques transferred by elements $\mathrm{e}$ and $f$ respectively and $\omega_{\mathrm{e}}$ and $\omega_{\mathrm{f}}$ are the angular velocities of those elements. By considering the differential as an isolated unit, the summation of torque's and powers can be equated to zero. This is shown in the following equations:

$$
\begin{gathered}
\sum T=T_{d}+T_{e}+T_{f}=0 \\
\sum P=T_{d} \varpi_{d}+T_{e} \varpi_{e}+T_{f} \varpi_{f}=0
\end{gathered}
$$

Solving the preceding equations simultaneously gives

$$
\Gamma=\frac{\varpi_{e}\left(\varpi_{f}-\bar{\varpi}_{d}\right)}{\bar{\omega}_{f}\left(\bar{\omega}_{d}-\bar{\varpi}_{e}\right)}
$$

or

$$
\Gamma=\frac{r(1-R)}{1-r}
$$

where

$$
r=\frac{\varpi_{e}}{\varpi_{d}}
$$




$$
R=\frac{\bar{\sigma}_{d}}{\bar{\sigma}_{f}}
$$

$\Gamma$ can now be derived for configuration 3.2 by finding $r$ and $R$ by inspection. Since $\omega_{\mathrm{d}}$ and $\omega_{\mathrm{f}}$ by definition are equal to the input and output angular velocities respectively, $\mathrm{R}$ is equivalent to $\omega_{\text {in }} / \omega_{\text {out }}$. For configuration 3.2, $\omega_{\text {in }} / \omega_{\text {out }}$ was derived and shown in Equation 3.7. By inspection of Figure $3.2 \omega_{\mathrm{e}}$ is equivalent to $\omega_{\mathrm{c}}$. Solving Equation 3.4 for $\omega_{\mathrm{c}} / \omega_{\mathrm{in}}$ results in Equation 3.27 which is equivalent to $\mathrm{r}$ for configuration 3.2.

$$
r=-\frac{\gamma_{c}}{\gamma_{h}}
$$

Substituting Equation 3.7 and 3.27 into Equation 3.24, allows $\Gamma$ to be calculated for this configuration. Using arbitrary values of $\gamma_{\mathrm{g}}=1.5, \gamma_{\mathrm{c}}=.32$, and varying $\gamma_{\mathrm{h}}$ between 1 and 5 an example curve for $\Gamma$ vs. $\gamma_{\mathrm{h}}$ can be plotted, as illustrated in Figure 3.7. 


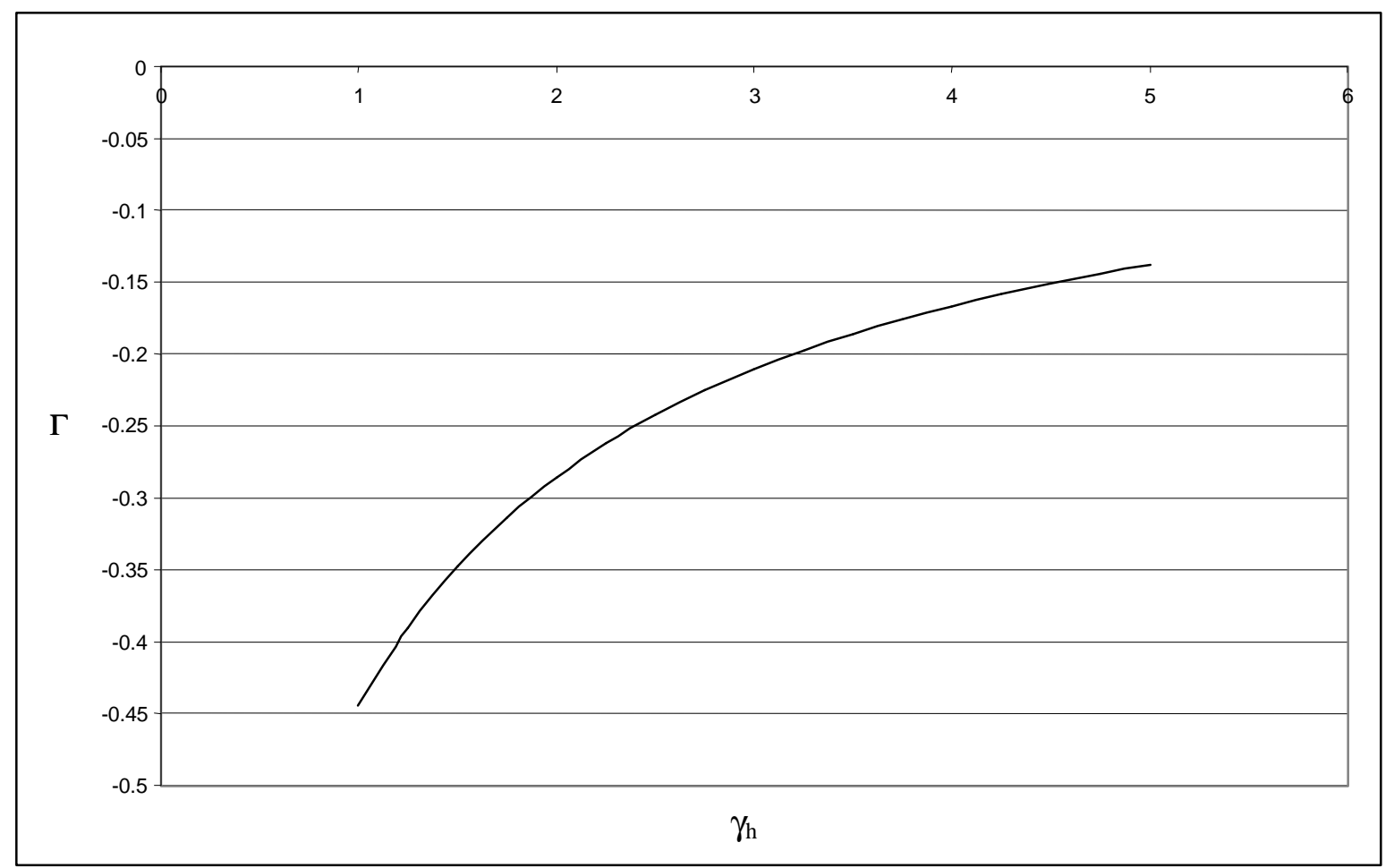

Figure 3.7: $\Gamma$ vs. $\gamma_{\mathrm{h}}$ for configuration 3.2 using arbitrary values.

Several issues can be addressed once $\Gamma$ is calculated for a configuration. First, if $\Gamma$ is positive, then power flows into or out of the differential through both elements e and $f$. This is a power re-circulation case, where power is actually flowing backwards through the variable element. If $\Gamma$ is negative, power flows into the differential through one of the elements (e or f) and out through the other. This is a power split case, since element $\mathrm{f}$ is always the output in an input coupled variable shunt transmission. Since $\Gamma$ is the percentage of power that flows through the branch element, $\Gamma$ greater than one or less than negative one means that there is a parasitic power flow. This is unsuitable because it means a variable element would have to be large enough to handle power greater then the input power. By using arbitrary values, plots of $\Gamma$ vs. $\gamma_{\mathrm{h}}$ were created for each configuration and can be found 
in Appendix A. The curves illustrate the trend of $\Gamma$ for each configuration, and allow the designer to compare $\Gamma$ of different configurations without actually using specific numbers.

\subsubsection{Calculation of Gamma with Power Loss}

Calculating $\Gamma$ for the ideal case gives a good estimate of how the power flows in the system but does not give an adequate calculation of the power that is supplied to the variable element. The power supplied to the variable element is higher than the power circulated in the planetary gear train, due to the efficiency of the variable element. This case is illustrated below in Figure 3.8. This power $\left(\mathrm{Po}_{\mathrm{i}}\right)$ affects the sizing of the variable element and the overall efficiency of the transmission. To determine $\mathrm{Po}_{\mathrm{i}}$ the power balance of the system is determined. The method of deriving the power balance is presented here using Configuration 3.2 as an example.

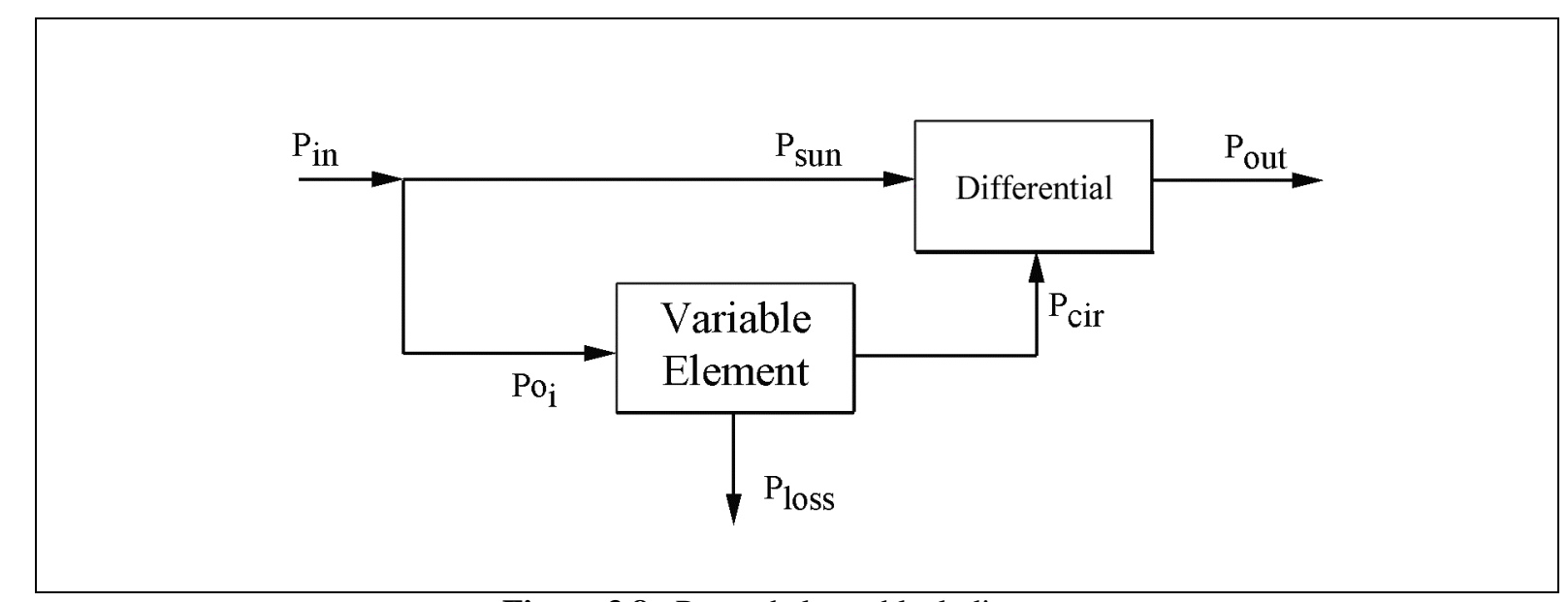

Figure 3.8: Power balance block diagram.

To develop the power balance for this system, the equations that govern the powers are determined from inspection of Figure 3.8. They are:

$$
\begin{aligned}
& \mathrm{P}_{\text {sun }}=\mathrm{P}_{\text {in }}+\mathrm{Po}_{\mathrm{i}} \\
& \mathrm{P}_{\text {cir }}=\mathrm{Po}_{\mathrm{i}}-\mathrm{P}_{\text {loss }}
\end{aligned}
$$




$$
\mathrm{P}_{\text {out }}=\mathrm{P}_{\text {sun }}+\mathrm{P}_{\text {cir }}
$$

The equation for $\mathrm{P}_{\text {loss }}$ using the definition of efficiency is

$$
\mathrm{P}_{\text {loss }}=\mathrm{Po}_{\mathrm{i}}(1-\eta)
$$

where $\eta$ is the efficiency of the variable element. $P_{\text {in }}$ is known from the specification of the applications engine. If $\mathrm{Po}_{\mathrm{i}}$ is found, then all the other powers can be solved. $\mathrm{Po}_{\mathrm{i}}$ can be expressed as

$$
\mathrm{Po}_{\mathrm{i}}=\mathrm{To}_{\mathrm{i}} \omega_{\mathrm{in}}
$$

In this equation $\omega_{\text {in }}$ is known and $\mathrm{To}_{\mathrm{i}}$ can be found by manipulating equations from section 1.3.2. The first step in deriving an expression for $\mathrm{To}_{\mathrm{i}}$ is to substitute $\gamma_{\mathrm{g}}$ and $\gamma_{\mathrm{c}}$ into equation 3.13 and solve for $\mathrm{To}_{\mathrm{r}}$. This yields:

$$
T_{\text {out }}=\frac{T o_{r}}{\gamma_{c}\left(\frac{1}{\gamma_{g}}+1\right)}
$$

Substituting this equation into 3.14 produces:

$$
T o_{i}=T_{i n}-\frac{T o_{r}}{\gamma_{c}\left(\gamma_{g}+1\right)}
$$

Rewriting equation 3.15 taking into account the efficiency of variable element results in the following equation:

$$
\eta \gamma_{h} T o_{i}=T o_{r}
$$

Substituting equation 3.35 into 3.34 and solving for $\mathrm{To}_{\mathrm{i}}$ results in an expression which can be used to solve for $\mathrm{To}_{\mathrm{i}}$. This expression is:

$$
T o_{i}=\frac{T_{i n}}{1+\frac{\eta \gamma_{h}}{\gamma_{c}\left(\gamma_{g}+1\right)}}
$$


Now that there is an equation for $\mathrm{To}_{\mathrm{i}}$ all of the powers in the system can be determined and a plot of the power balance can be created. Using the equations presented, a power balance curve for configuration 3.2 is illustrated in Figure 3.9. Arbitrary values of $\gamma_{\mathrm{g}}$ $=1.5, \gamma_{\mathrm{c}}=.32, \mathrm{P}_{\mathrm{in}}=425 \mathrm{hp} @ 2100 \mathrm{rpm}$ and varying a $\gamma_{\mathrm{h}}$ between 1 and 5 where used. For this example, an efficiency for the variable element of $80 \%$ was arbitrarily used.

The power balance illustrates several important design considerations. First, it allows the designer to size the variable element based on the maximum $\mathrm{Po}_{\mathrm{i}}$. Second, it illustrates that the efficiency of the variable element directly affects the size of the variable element and its effect should not be neglected. Comparing the ideal $\Gamma$ from the last section to the power balance presented here, further supports this since there is a $5 \%$ difference in the input

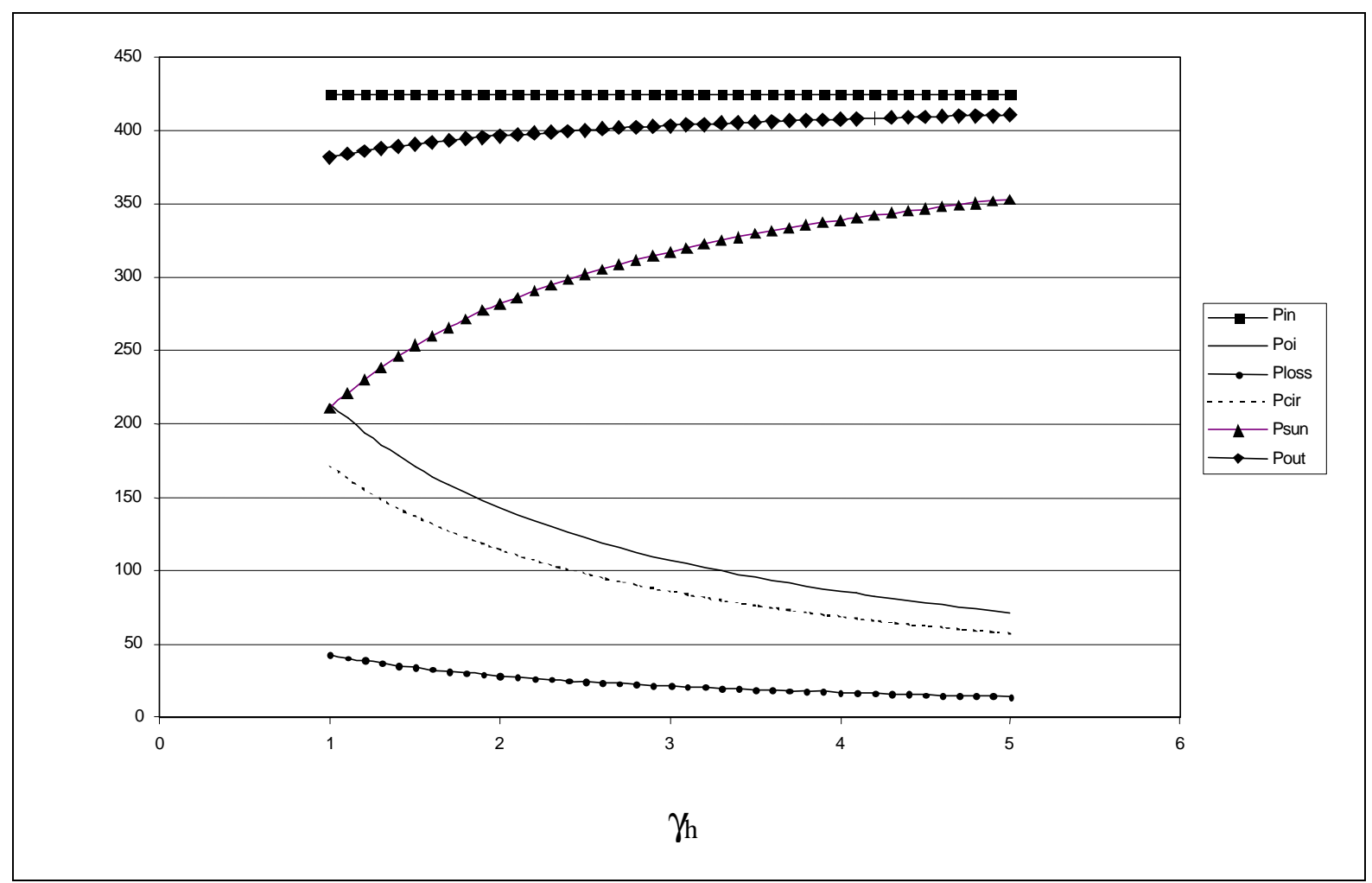

Figure 3.9: Example power balance curves for configuration 3.2. 


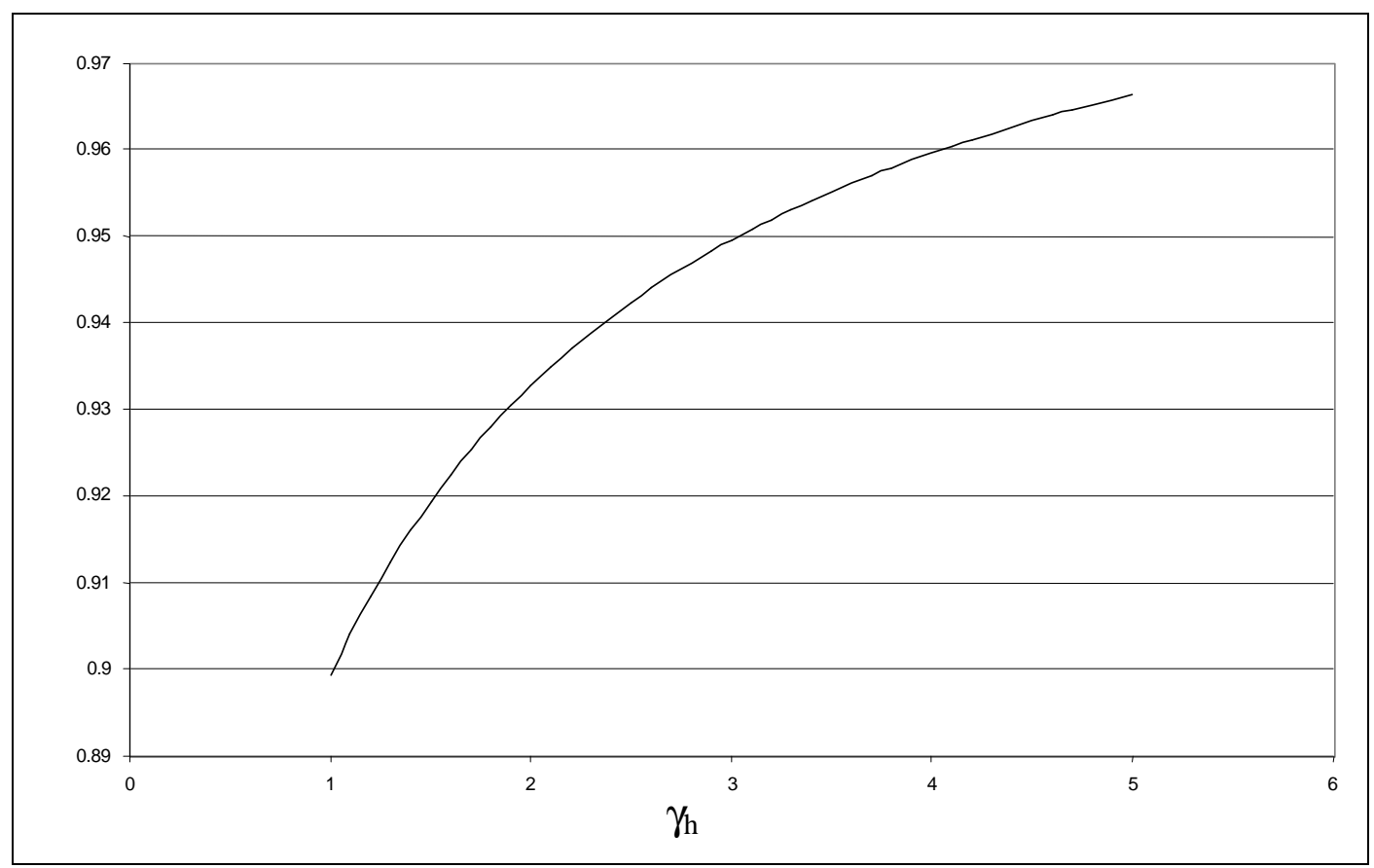

Figure 3.10: Estimated overall efficiency for example configuration 3.2.

power to the variable element. Therefore, the most efficient variable element not only increases the overall efficiency of the transmission but also decreases the size of the variable element and thus the overall size of the transmission. The last concept the power balance allows the designer to investigate is the overall efficiency of the transmission. The main power loss in the transmission is due to the efficiency of the variable element. Therefore, the overall efficiency can be estimated by dividing the power out by the power in across the entire operating range of the transmission.

\subsection{Gamma - Span Relationship}

The methods of calculating $\Gamma$ and the span for a given configuration have been presented, but before any design can begin the relationship between the two must be understood. Changing either of these has an effect on the other. The general relationship 
between the two is that the larger the span of a configuration, the larger $\Gamma$. This basic relationship is described using graphical analysis of an example.

Since equations for the span and $\Gamma$ where derived for Configuration 3.2, this configuration will be the used to examine the relationship between $\Gamma$ and the span. Inspection of the equations (Equations 3.19, 3.7 and 3.27) reveal five variables: $\gamma_{\mathrm{g}}, \gamma_{\mathrm{c}}, \gamma_{\mathrm{h}}, \gamma_{\mathrm{hL}}$, and $\gamma_{\mathrm{hH}}$. Two of these are determined by the designer, these are $\gamma_{\mathrm{g}}$ and $\gamma_{\mathrm{c}}$. The others are defined by the variable element. There is an infinite combination of these two variables, that result in the same span for this configuration. This is illustrated in Figure 3.11, where the combinations of $\gamma_{\mathrm{g}}$ and $\gamma_{\mathrm{c}}$ where found for a span of 1.5. This was done, by solving Equation 3.19 for $\gamma_{c}$ yielding:

$$
\gamma_{c}=\frac{\gamma_{h L} \gamma_{h H}(1-\text { span })}{\left(\gamma_{h H} \operatorname{span}-\gamma_{h L}\right)\left(\gamma_{g}+1\right)}
$$

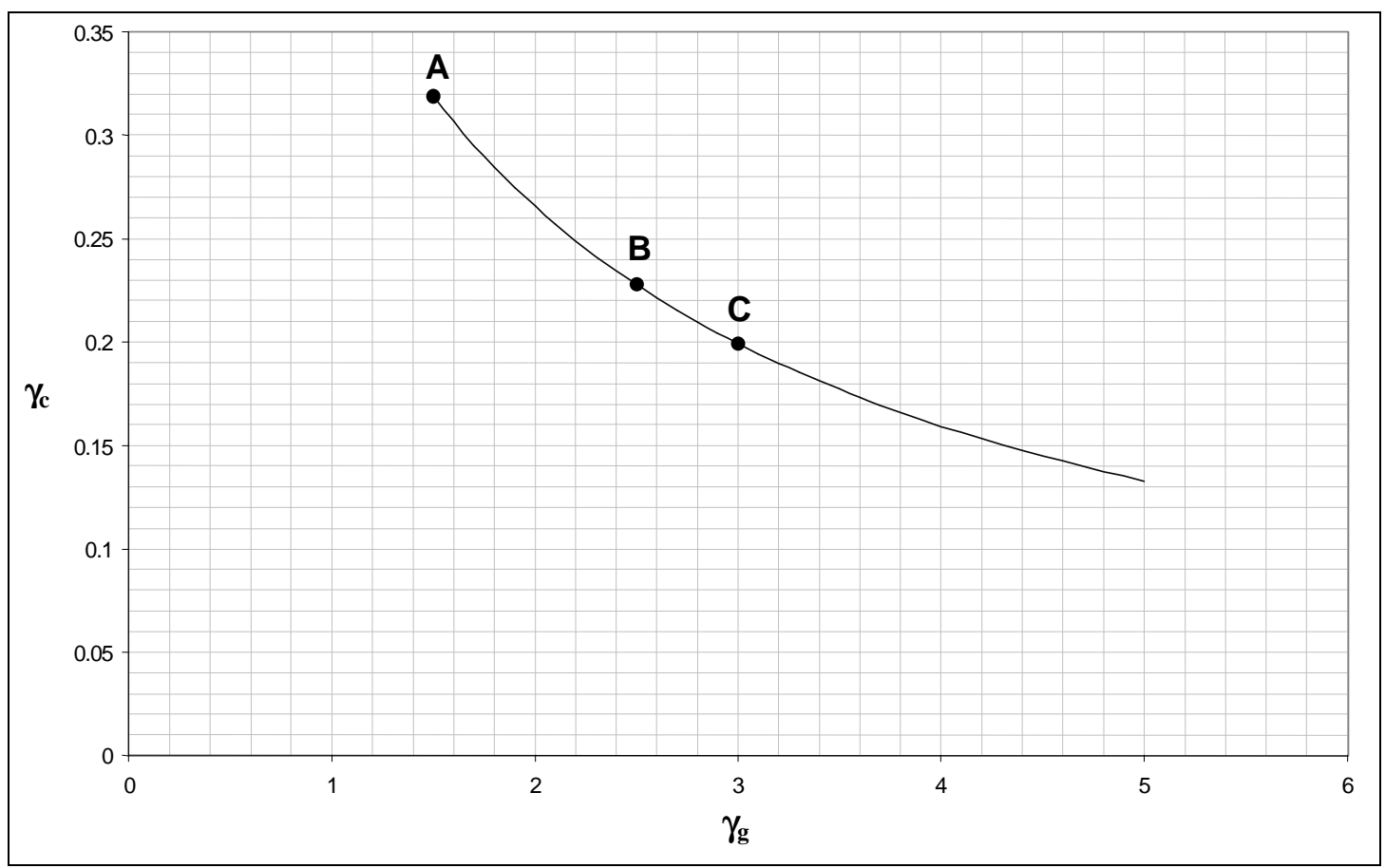

Figure 3.11: Possible combinations of $\gamma_{\mathrm{g}}$ and $\gamma_{\mathrm{c}}$ for configuration 3.2 with a span of 1.5. 
Then by setting the span $=1.5, \gamma_{\mathrm{hH}}=1, \gamma_{\mathrm{hL}}=5$, and varying of $\gamma_{\mathrm{g}}$ and solving for $\gamma_{\mathrm{c}}$, several sets of data were calculated and used for the plot.

Since there are several combinations of $\gamma_{\mathrm{g}}$ and $\gamma_{\mathrm{c}}$ that yield the same span, their affects on $\Gamma$ need to be understood to determine which of the combinations to use for a given application. To determine this, Equation 3.37 is substituted into Equations 3.7 and 3.27. The results of these substitutions are shown below.

$$
\begin{aligned}
\frac{\omega_{\text {in }}}{\omega_{\text {out }}} & =-\frac{\gamma_{g} \gamma_{h}}{\gamma_{h}+\frac{\gamma_{h L} \gamma_{h H}(1-\operatorname{span})}{\left(\gamma_{h H} \operatorname{span}-\gamma_{h L}\right)\left(\gamma_{g}+1\right)}} \\
r & =\frac{\gamma_{h L} \gamma_{h H}(1-\operatorname{span})}{\left(\gamma_{h H} \operatorname{span}-\gamma_{h L}\right)\left(\gamma_{g}+1\right) \gamma_{h}}
\end{aligned}
$$

$\Gamma$ is now in terms of $\gamma_{\mathrm{g}}$ and span. The effect of $\gamma_{\mathrm{g}}$ can be illustrated by calculating $\Gamma$ while varying $\gamma_{\mathrm{g}}$ and fixing span and $\gamma_{\mathrm{h}}$. This was done for several values of span and the data are illustrated in Figure 3.12. This figure shows that the combination of $\gamma_{\mathrm{g}}$ and $\gamma_{\mathrm{c}}$ results in the same $\Gamma$ for any span. A curve can be plotted (Figure 3.13) which illustrates the relationship between the span and $\Gamma$ of configuration 3.2. This figure is in terms of the absolute value of $\Gamma$ and percentage of ratio coverage of the variable element. For example, if the variable element has a span of 5 and a span of 5 is required by the application then $100 \%$ of the power will flow through the variable element. The method of creating this plot can be used to create similar plots for each configuration. The plots of $\Gamma$ vs. the ratio coverage are very useful and will be utilized in the design procedure. 


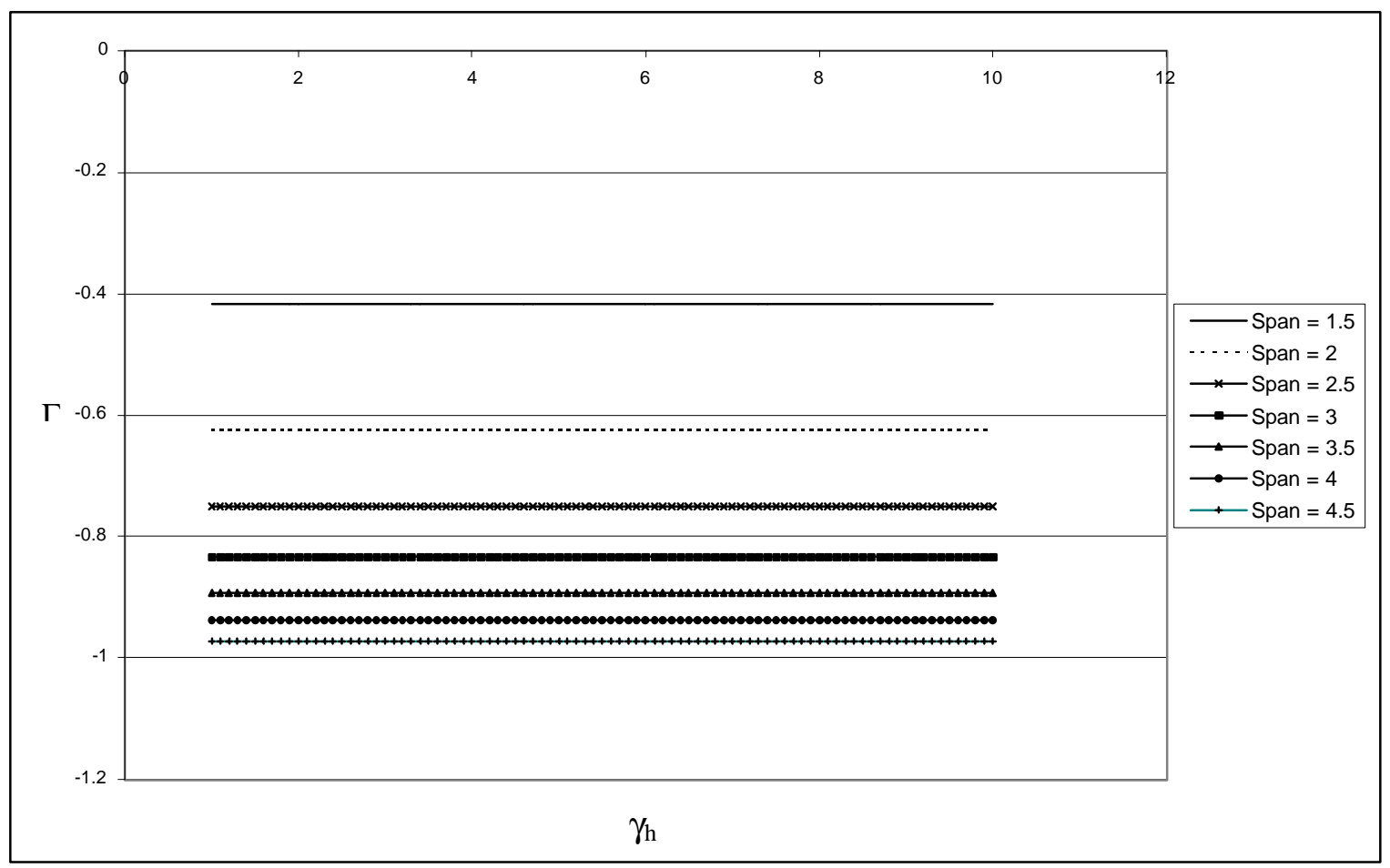

Figure 3.12: $\Gamma$ vs. $\gamma_{\mathrm{g}}$ for configuration 3.2 with various spans.

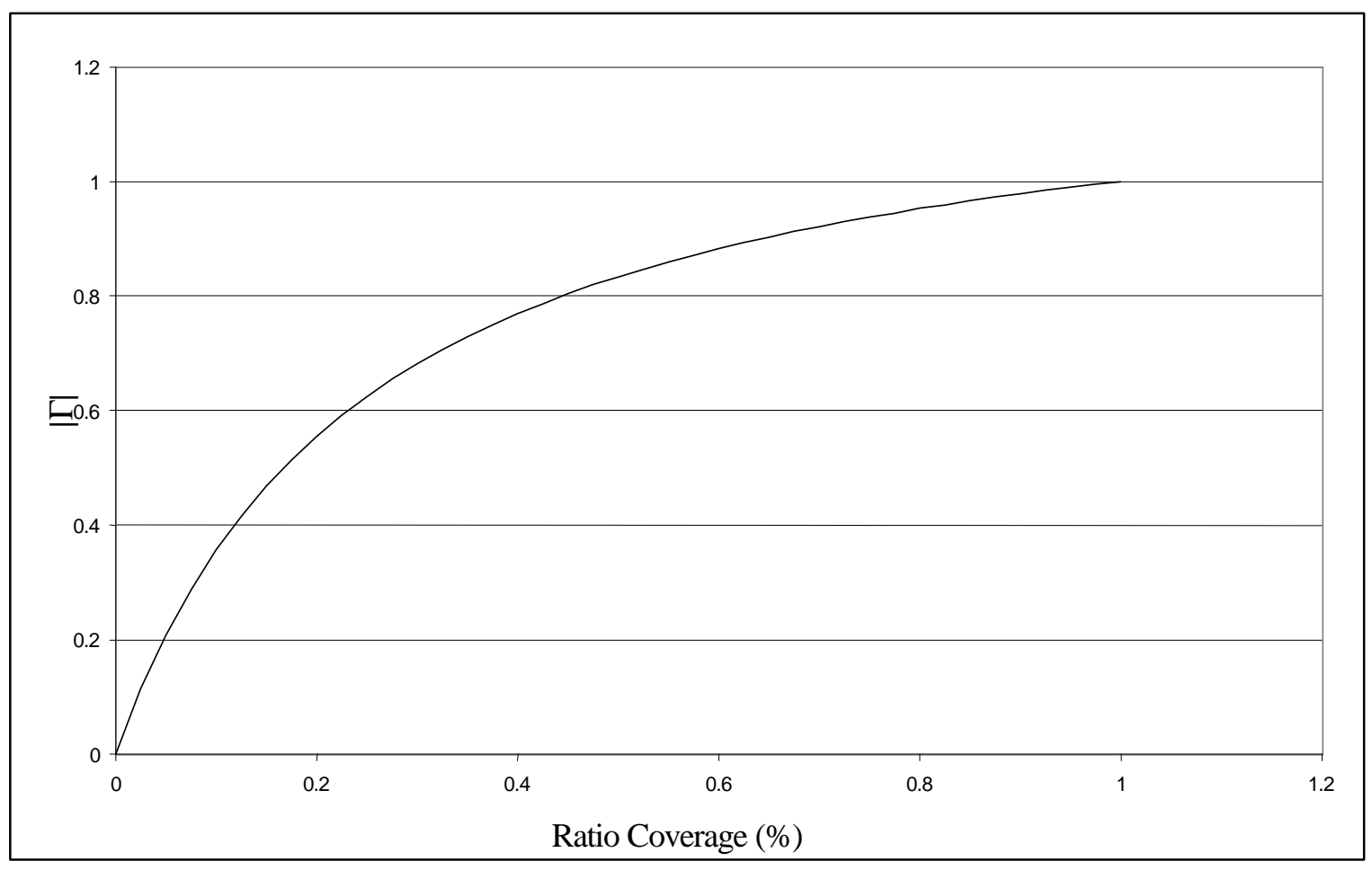

Figure 3.13: Absolute $\Gamma$ vs. the ratio coverage of the variable element for configuration 3.2 
Though the combinations of $\gamma_{\mathrm{g}}$ and $\gamma_{\mathrm{c}}$ which result in a certain span also equate to the same value of $\Gamma$, the different combinations have a large affect on the input to output angular velocity ratios of a configuration. Three combinations of $\gamma_{\mathrm{g}}$ and $\gamma_{\mathrm{c}}$ from Figure 3.11 (points A, B, and C) where used to plot the input to output velocity ratios for these points. This plot, Figure 3.14, shows that even though $\Gamma$ is the same, the velocity ratios covered are different. This effect can be considered in the design procedure.

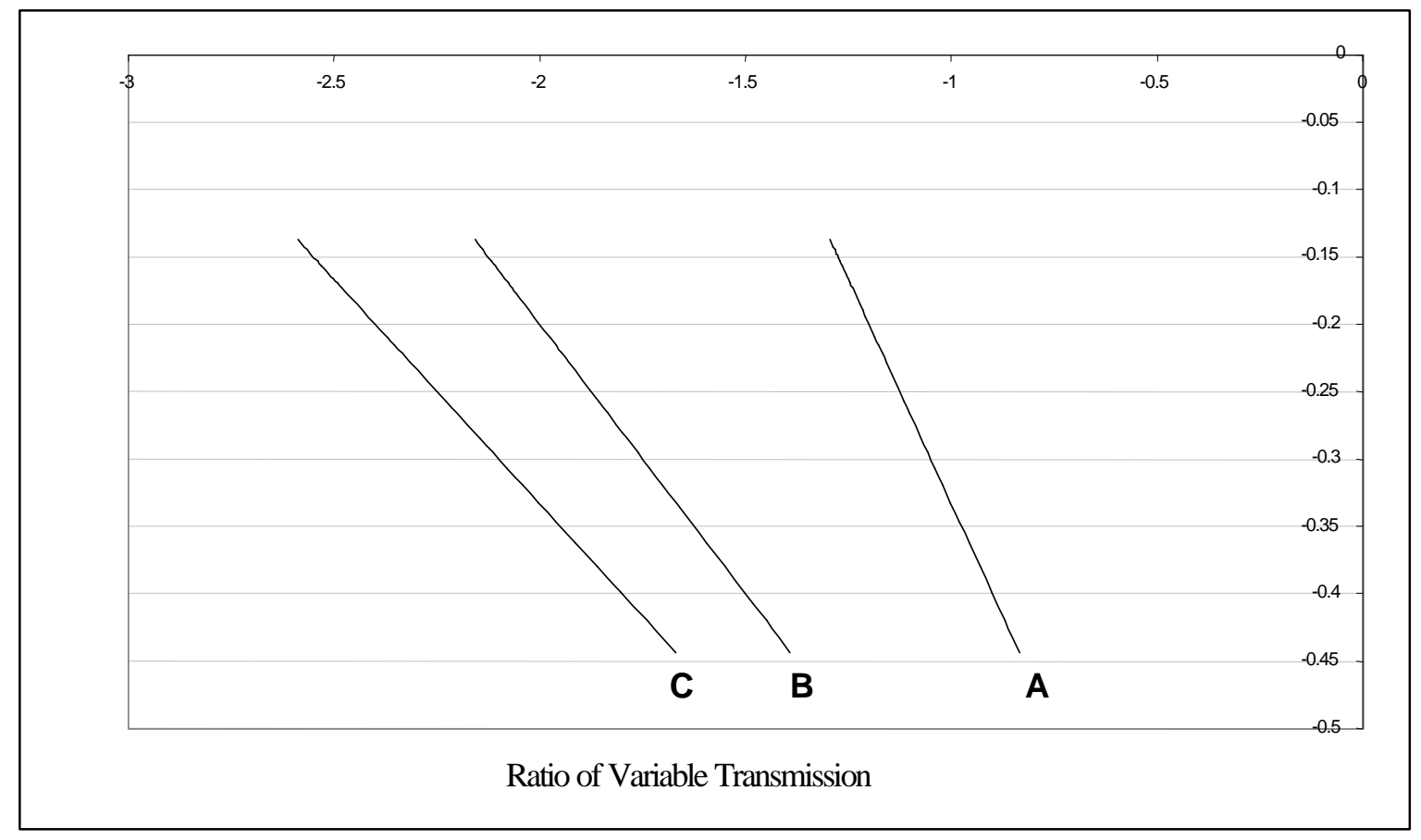

Figure 3.14: Comparison of the three combinations of $\gamma_{g}$ and $\gamma_{c}$ from Figure 3.10. 


\subsection{Variable Element}

Any shaft to shaft continuously variable drive could be used as the variable element in a PS-CVT. For this study, two types of hydraulic elements are considered. Both are used in examples of the design procedure. These are hydrodynamic couplings and hydrostatic drives. Information was acquired on both types, and used below to describe them in detail.

\subsubsection{Hydrodynamic Couplings}

The first variable element used in an example of the design procedure is a hydrodynamic coupling. In this report, information from catalogs provided by the company Voith Transmissions Inc. were used. The type of variable speed turbo coupling selected is illustrated in Figure 3.15 (Voith 1997).

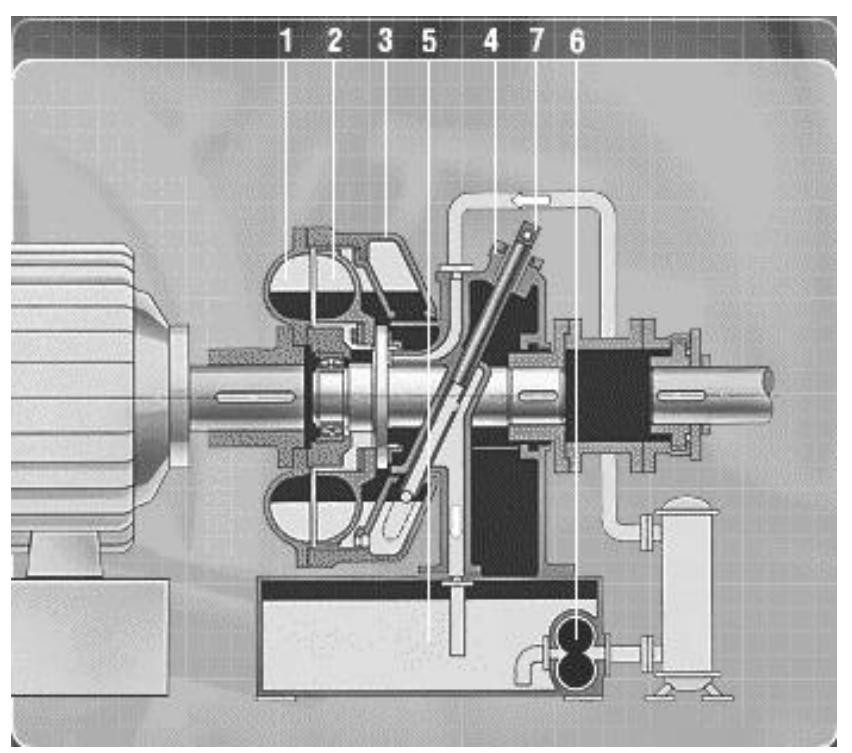

Figure 3.15: Voith SVN hydraulic variable speed turbo coupling.

The hydraulic coupling is a fluid coupling which transmits the power input by the mass forces of a fluid which is circulated in a closed system between and impeller on the driving shaft and a runner on the driven shaft. The impeller and runner are labeled 1 and 2 respectively in Figure 3.15. The oil fill in the working compartment can be varied between 
completely filled and drained. The amount of oil in the working compartment determines the speed at the output side of the coupling and is dependent upon the position of the scoop tube. The scoop tube is labeled 7 in the figure. The Voith SVN hydraulic coupling is available in sizes from 100 to $1000 \mathrm{~kW}$ (Voith 1997). The actual selection of the coupling is associated with operational ranges required for the transmission.

The performance characteristics of these type of hydraulic couplings are illustrated in Figure 3.16 (Voith 1997), in which a "decreasing" torque curve for the output shaft is illustrated. The speed ratio refers to the output-to-input velocity ratio $\left(\omega_{\text {out }} / \omega_{\text {in }}\right)$ as does the relative torque $\left(\mathrm{T}_{\text {out }} / \mathrm{T}_{\text {in }}\right)$. The family of curves indicates the scoop tube position changing from minimum (almost horizontal) to maximum (almost vertical).

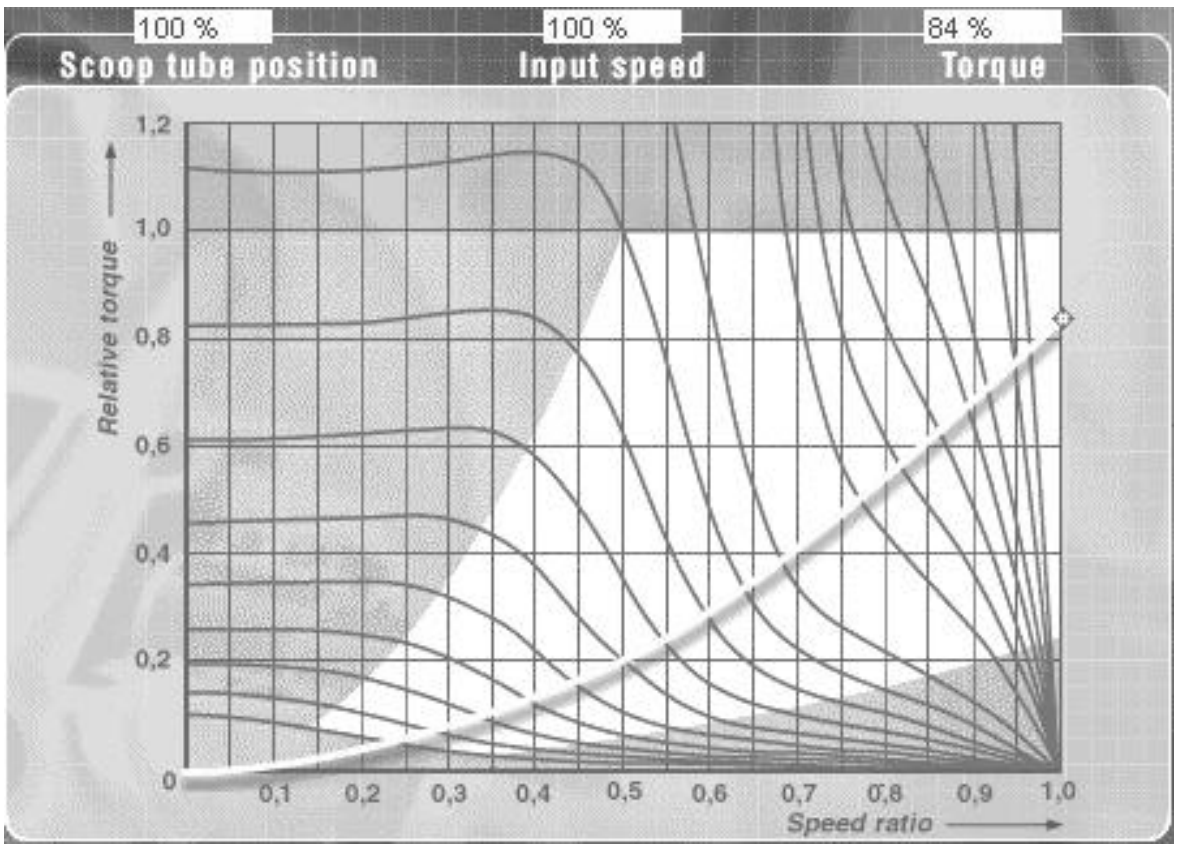

Figure 3.16: Relative torque versus speed ratio $\left(\omega_{\text {out }} / \omega_{\text {in }}\right)$ for hydrodynamic coupling from the Voith Catalog. 


\subsubsection{Hydrostatic Drives}

There were several companies that manufacture hydrostatic drives, and in general they have similar features. For this design study information provided by Sauer-Sundstrand was used. A typical hydrostatic drive is illustrated in Figure 3.17 (Sauer-Sundstrand 1997).

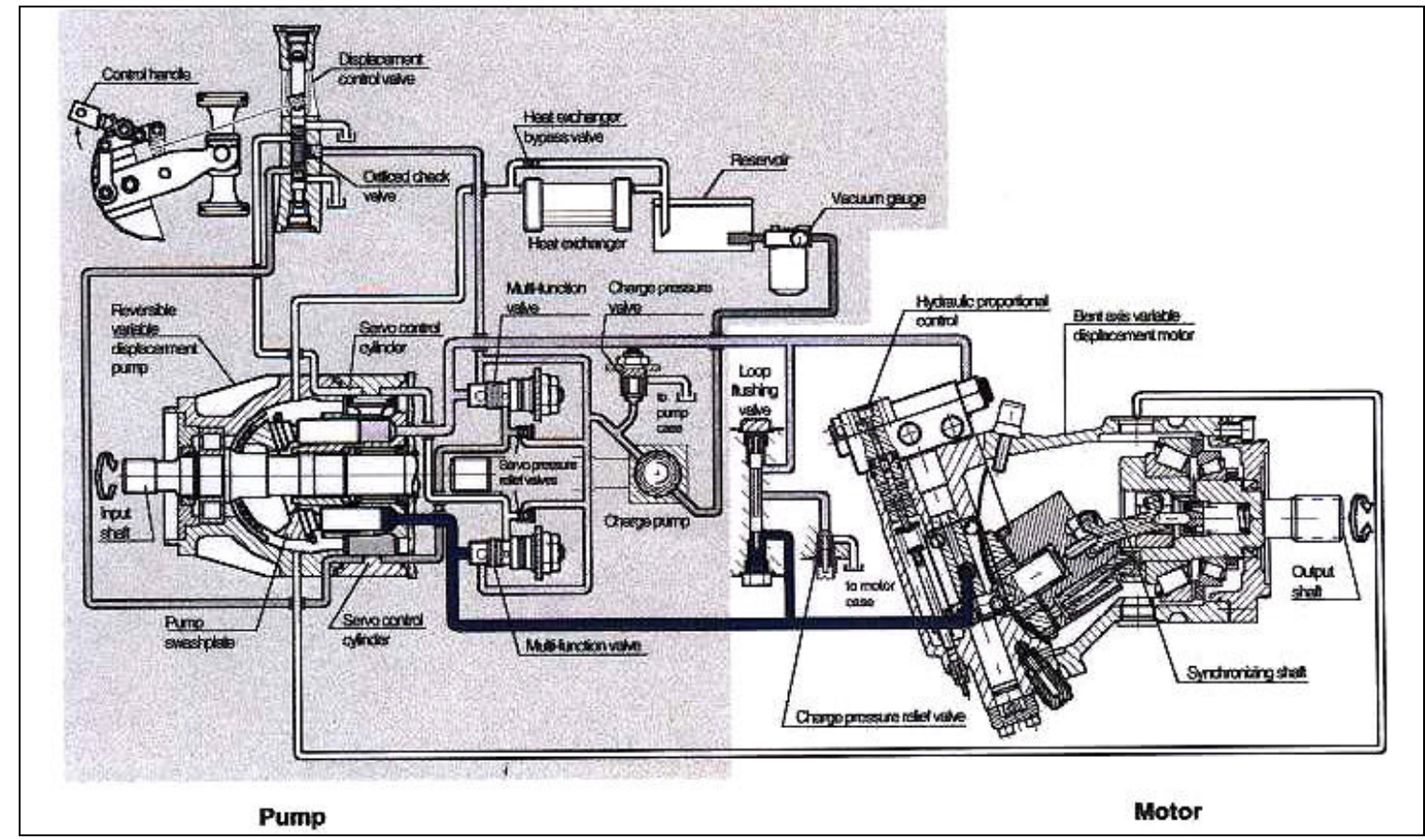

Figure 3.17: Hydrostatic transmission configuration with a variable pump and motor.

A hydrostatic drive consists of 2 main components a hydraulic pump and a hydraulic

motor. Each of these can have either a fixed or variable displacement. These 4 components can be combined to create 4 different circuits, which exhibit different performance characteristics. It was determined that a variable displacement pump driving a variable displacement motor was the best circuit for this application (Bretz 1998).

In general these types of elements are more efficient overall and more compact than torque converters and hydrodynamic couplings. The efficiency of a hydrostatic drive is based on three factors. These factors are the percentage of maximum displacement, percentage of rated continuous speed, and system pressure. Figure 3.18 shows a typical 
efficiency plot for a Series 51 Sauer-Sundstrand motor. Note that this is only for maximum displacement.

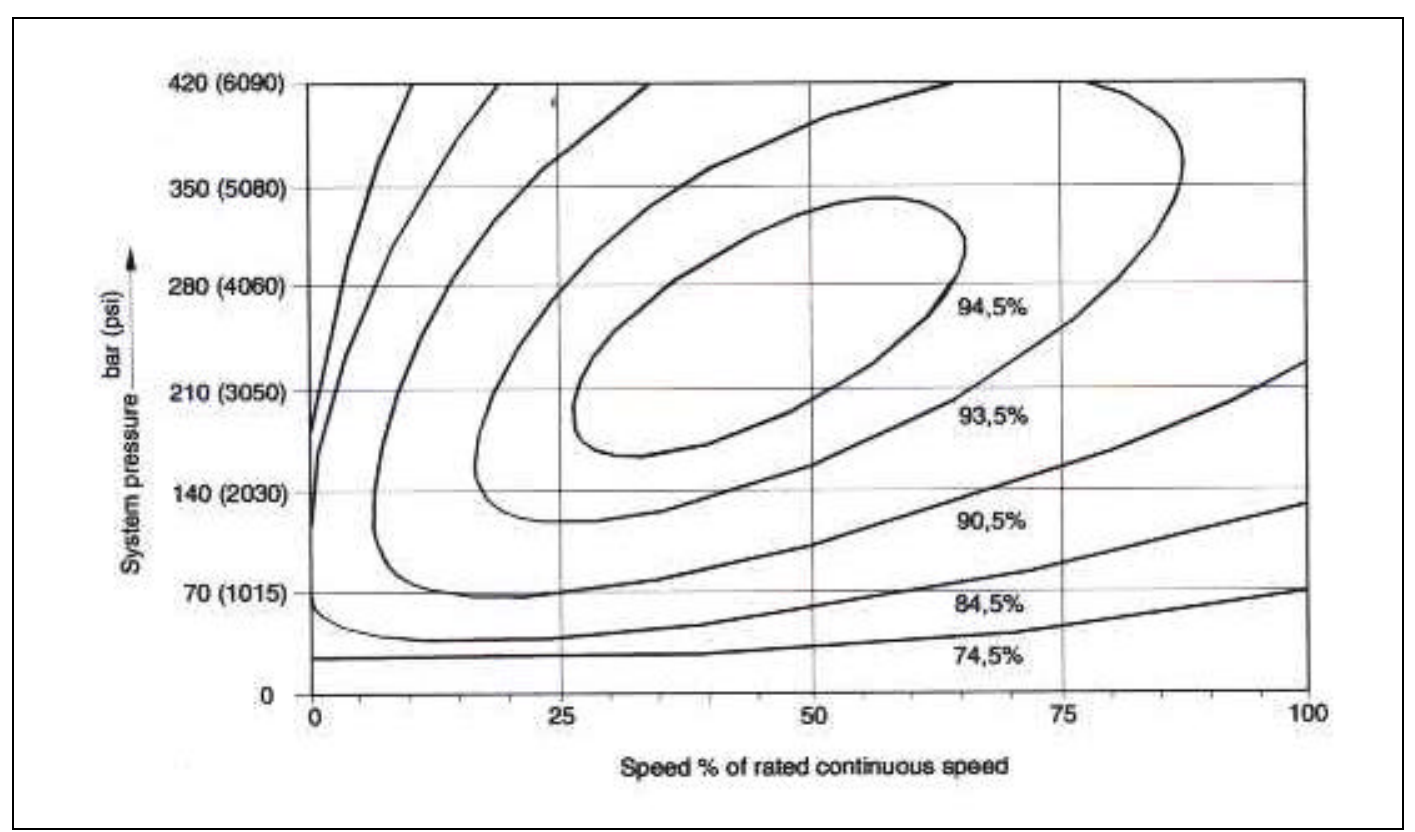

Figure 3.18: Overall efficiency at maximum displacement for Sauer-Sundstrand Series 51 motor.

To determine the pump and motor combination that would best suit the two applications, Sauer-Sundstrand was contacted. Due to the large number of variables in selecting a pump and motor combination and obtaining efficiency data for the configurations Sauer-Sundstrand used computer modeling. Using input and output information provide by the theoretical calculations Sauer-Sundstrand was able to match a hydrostatic drive to meet the needs of the example application. The specifics are presented later in the example of the conceptual design procedure. 


\section{Chapter 4 Conceptual Design Procedure}

\subsection{Introduction}

Given the engine characteristics, output requirements, and eligible configurations, how does one design the gear train? This involves determining the gear ratios, sizing the gears and selecting a variable element, and is usually the result of a conceptual design procedure. This chapter outlines the steps required to complete a conceptual design for a multistage power split continuously variable transmission for an industrial application.

\subsection{Conceptual Design Procedure}

The complete design procedure has been outlined in flow chart form, Figure 4.1. The flow chart contains all the necessary steps required to develop a conceptual design of a multistage PS-CVT. This process starts with defining the input variables, which are the input power, input speed range, and output speed requirements, and ends with selected gear ratios and sizes, specification of variable element and theoretical efficiency of the design. The design procedure contains 14 steps, which are numbered in Figure 4.1. To help the reader follow the steps of the flow chart in the description below, step numbers are included in parentheses at the beginning of each step description.

To begin the conceptual design procedure, the specifications of the application must be defined (1). The values that are required are: input power, the operating speed range of the input device, and the transmission output speed range. Once these specifications are determined, the actual process can begin. The first step in the procedure is to determine the maximum allowable $\Gamma$ (2). The larger the allowable $\Gamma$, the larger the variable 


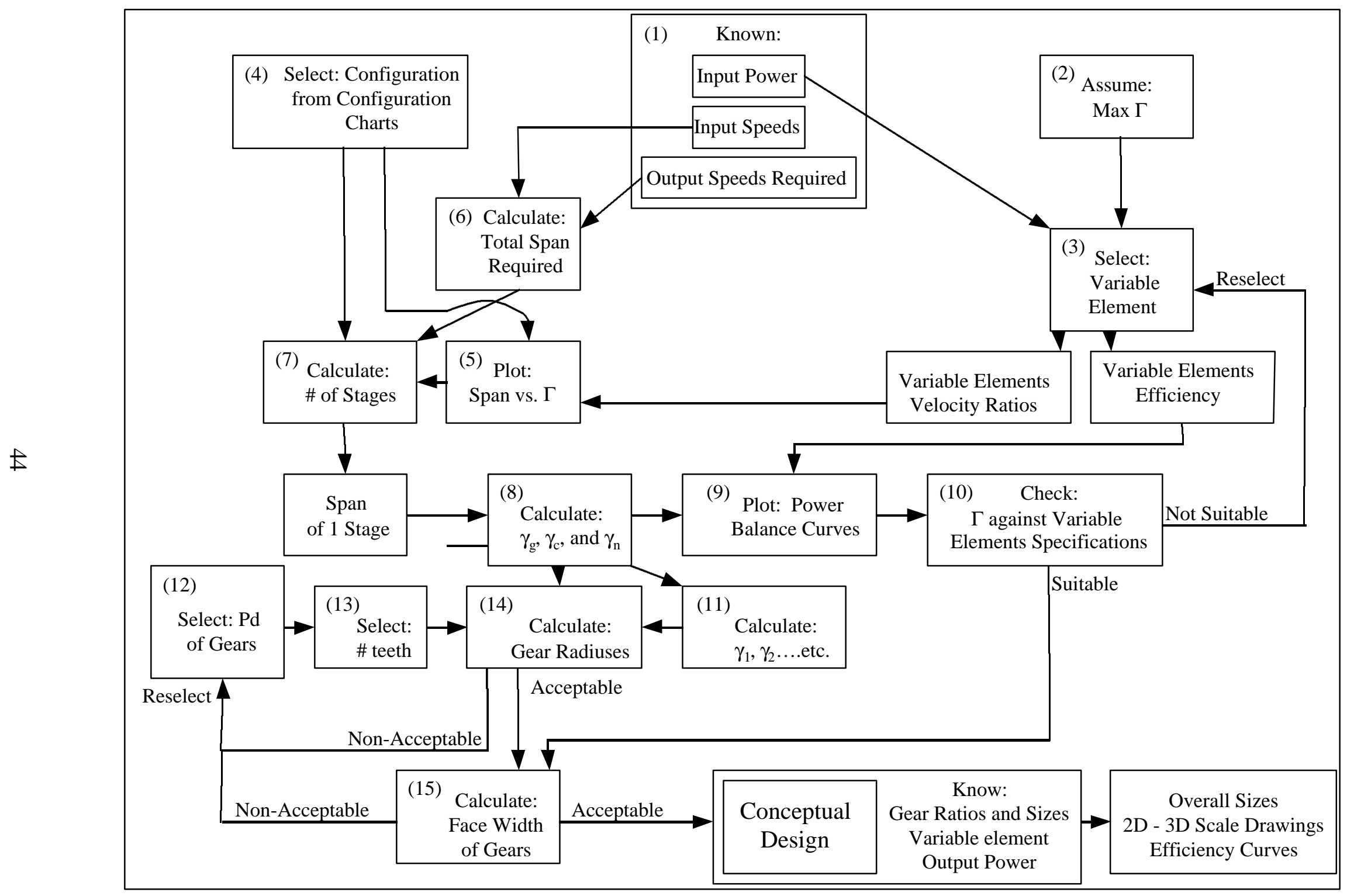

Figure 4.1: Conceptual design procedure flow diagram. 
element. Smaller $\Gamma$ result in the less power flow through the variable element and therefore, a higher overall efficiency for the transmission. Based on the sizes of the power capacities of the variable elements available and the total input power the designer can estimate a maximum $\Gamma$. After the maximum $\Gamma$ has been determined, it can be used in combination with the input power to select a variable element (3). An initial variable element is selected from available literature (this selection will be checked later in the procedure). Selection of the variable element results in specification of the velocity ratio range and the efficiency of the variable element.

The next selection made by the designer, is the configuration to use for the conceptual design (4). The designer uses the charts, found in Appendix A, and selects one of the sixty combinations based on the $\Gamma$ and velocity ratios curves which meet the requirements of the application. Once this step is completed, the equations for the properties of that configuration can be used with the variable element's velocity ratio range to plot a span vs. $\Gamma$ curve (5). From this curve, the allowable span for a stage due to the maximum $\Gamma$ is determined. Next, using the input speed range and the transmission output speed range, required the overall span of the transmission can be determined (6). Using the allowable span for a stage, and the overall span required, the number of stages that is needed can be determined (7). Starting with one stage, the number of stages is increased until the span required for a stage is less then the allowable span. If the span required for a given number of stages is slightly larger then the allowable span and does not warrant another stage, the maximum $\Gamma$ can be slightly increased. This can be done without returning to step 2 to repeat all the steps as it will be checked later in the design procedure. 
The span required for one stage has been calculated and the process of determining gear ratios and sizes can begin. The first ratios that are calculated are the ratios of the control unit $\left(\gamma_{c}\right.$ and $\left.\gamma_{n}\right)$ and the planetary gear train $\left(\gamma_{g}\right)(8)$. These can be determined using the relationships developed in the previous chapter. The next step is to plot the power balance curves. This is done using the gear ratios from step 8 and the efficiency of the variable element which was a result of step 3. The main product of this plot is a value of the actual power that flows through the variable element $(\Gamma)$. This actual $\Gamma$ is used as a check to determine if the specified variable element can handle the maximum calculated power from the power balance curves (10). If the specified variable element is not suitable then a new one must be selected and the process repeated to this point until a suitable one is found.

The next step is to determine the ratios for the external gearbox, $\gamma_{1}, \gamma_{2}$, etc. (11). These are the ratios which are used to vary the stages, and thus the number of stages determines the number of ratios which need to be calculated. The first ratio is determined by making the low speed ratio of stage one equivalent to the lowest speed ratio required. Then the second stage low speed ratio is made equivalent to the high speed ratio of stage two. This process is repeated until all the ratios have been calculated. If this process is done correctly the high speed ratio of the last stage should match the high speed requirement of the transmission. When these values are determined, all of the transmission gear ratios are known. 
The next two steps, determining the diametral pitch $(\mathrm{Pd})$ and the number of teeth for each gear, determine the sizes (diameter and face width) of the gears. Changing either of these values effects the sizes of the gears. The process to determine the diametral pitch and number of teeth for each gear is iterative. The acceptability of the sizes is left to the discretion of the designer.

To start the iterative process of sizing the gears, a diametral pitch is assumed (12). The diametral pitch may be different for each gear ratio. Generally the larger the diametral pitch the narrower the face width for the same diameter gear. Larger diametral pitches should be used for the gear box ratios because they transfer all the force through one set of teeth. The control gears and the planetary gear train may have smaller diametral pitch because they have three pairs of teeth in contact. The initial assumption will be checked. However, a good initial assumption will result in less iterations to complete the procedure. Regardless of the accuracy of the initial assumption, an acceptable diametral pitch will be found by following the process. After a diametral pitch is assumed, the number of teeth for each gear set is determined to match the desired ratio (13). The only constraint here is that gears sets on parallel shafts must have the same center distances. This can be checked with standard gear equations.

Once the diametral pitch and the number of teeth for each gear has been assumed, the radii for each gear can be calculated using standard gear equations (14). The acceptability of the values calculated for each gear is up to the discretion of the designer. The criteria used for the gears radii can be related to a number of factors, which are determined by the application. If the gear radii are not acceptable the diametral pitch or the number of teeth per gear must be adjusted. Once acceptable radii have been found for all gears, the gears face 
widths can be calculated using AGMA standards (Avallone and Baumeister 1996, Shigley and Mischke 1989). Power balance curves are used to determine the loads being transferred by each gear set. The acceptability of the gear face width is again, at the designer's discretion. When acceptable gear sizes have been determined the conceptual design procedure has been completed.

Several things have been determined in the conceptual design procedure. These are the gear ratios and sizes, the variable element, and the output power. Using this information the designer can: estimate the overall size of the transmission, develop $2 \mathrm{D}$ and $3 \mathrm{D}$ drawings, and produce efficiency curves. This information is useful in determining the feasibility of a multistage power split continuously variable transmission to fulfill the requirements of an application. 


\section{Chapter 5 Example Conceptual Design}

\subsection{Application Description}

In this chapter, the conceptual design procedure is applied to the development of a power split continuously variable transmission for an industrial application. In general, this process, may be used as the initial step in determining whether a PS-CVT is a viable alternative. To determine the feasibility for an application, the procedure addresses the following issues.

1. Can a power split configuration be found which meets the input/output speed and power requirements of the application?

2. Is the overall size of the transmission within the design requirement?

3. What is the estimated efficiency of the resulting PS-CVT design?

Once favorable results are obtained from the design procedure described here, transition to the refined design stage is made.

An industrial pump has been chosen for use as example of an industrial application. In the current pump system an electronically controlled automatic transmission is used to transmit power from the power source to the pump. The conceptual design procedure is utilized to determine if a PS-CVT is a feasible replacement option for this application. Two conceptual designs, utilizing different variable elements, are considered in the study. One uses a hydrodynamic coupling and a second one uses a hydrostatic drive. 


\subsection{Conceptual design using a hydraulic coupling}

The first step in the procedure was to define the application. The power source for the industrial pump is a $425 \mathrm{hp}$ diesel engine that operates between 1300 and $2100 \mathrm{rpm}$. Speed reduction in this system is achieved with a transmission and 8.6:1 worm gearing on the pump. This combination results in pump operation speeds of 47 to $244 \mathrm{rpm}$ and transmission output speeds of between 408 and $2100 \mathrm{rpm}$. The PS-CVT will be replacing a 4 speed automatic transmission with ratios varying from 3.19:1 to 1:1. The block diagram, Figure 5.1, illustrates the application requirements.

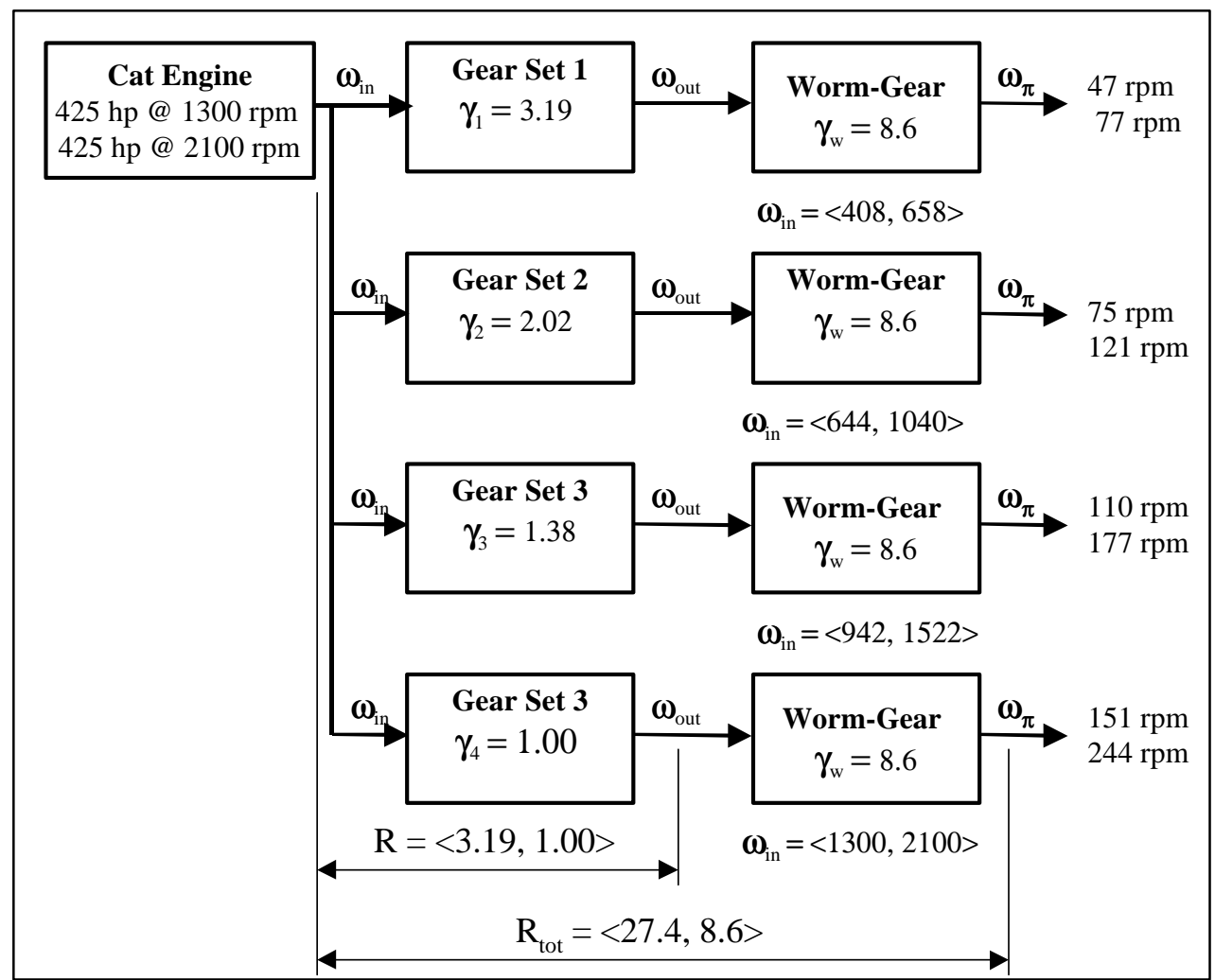

Figure 5.1: Block diagram of automatic transmission to be replaced in example application.

For step 2 of the procedure, a maximum power fraction of .5 was assumed implying that half of the input power will flow through the variable element. With this information, a coupling was selected from the Voith (1997) catalog, completing step three. A $\Gamma$ of .5 and a 
maximum input power of $425 \mathrm{hp}$, requires that the selected coupling have a capacity above $212 \mathrm{hp}$. The table in catalog (Voith 1997) indicates that the smallest unit that meets this requirement is a Voith SVN 316, which has a 316 hp capacity. The coupling velocity ratio range (range of $\gamma_{\mathrm{h}}$ ) varies between 5:1 to $1: 1$, and with an efficiency of approximately $1 / \gamma_{\mathrm{h}}$.

Step 4 consists of PS-CVT input-output configuration selection from the configuration charts found in Appendix A. Since a hydrodynamic coupling is utilized in this example, the $\Gamma$ range must fall between 0 and -1 , implying that the power is never regenerative. The inclusion of a reduction gearbox in the PS-CVT requires that the velocity ratio be negative such that the input and output of the entire transmission will rotate in the same direction. For this application, configuration 3.2 was selected. This configuration offers the advantages of a large, negative velocity ratio span while fulfilling the requirements for $\Gamma$, as stipulated above.

Step 5 utilizes the range of velocity ratios resulting from step three to construct a plot of the span- $\Gamma$ relationship. For this application, this relationship was the same as described in the example plot (Figure 3.13) presented in chapter 3. Calculation of the total span required for the application was accomplished by dividing the low speed ratio by the high speed ratio of the automatic transmission being replaced. This results in an overall span for this application of 3.19, completing step 6.

Step 7, involves the calculation of the number of stages required to cover the overall span for the application. This was accomplished by an iteration process using the following equation.

$$
\operatorname{span}_{i}=\sqrt[N S]{\operatorname{span}}
$$


In the equation, $\operatorname{span}_{i}$ is the span required for each stage, $N_{s}$ is the number of stages, and span refers to the overall span required for the application. Each iteration begins by assuming a number of stages and computing the span required for each stage using Equation 5.1. An additional $5 \%$ is added to the calculated span in order to account for discrepancies that occur downstream in the process when gears are selected to match theoretical values. The resulting span is then divided by the total ratio range of the variable element which produces a value referred to as the "ratio coverage" of the variable element. Once the ratio coverage is determined, the span- $\Gamma$ plot constructed in step 5, is utilized to ascertain the value of $\Gamma$ that corresponds to the span for the number of stages used. This value is then compared to the maximum allowable $\Gamma$ selected in step 2. If it is below the allowable value, then the number of stage is acceptable. If not, the iteration must be repeated using a larger number of stages. This iteration process is completed for the example configuration starting with a single stage and adding one stage with each iteration until a $\Gamma$ of less then .5 is obtained. For this application use of a single stage results in an overall span of 3.35 (after $5 \%$ is added), which corresponds to a coupling ratio coverage of $58 \%$. The $\Gamma$ value obtained from the span- $\Gamma$ curve, Figure 3.13 , was over $80 \%$, which is above the maximum allowable. Repeating the process with two stages, results in a value for $\Gamma$ of $59 \%$. With three stages, the span of each stage becomes 1.52 and the resulting value of $\Gamma$ was calculated to be $42 \%$ which is below the maximum allowable. The iteration process was then complete with three stages proving to be sufficient for the system requirements.

In step 8, the values for $\gamma_{\mathrm{g}}$ and $\gamma_{\mathrm{c}}$ were determined. This involves finding combinations of the two unknown variables that produce a span of 1.52 and was accomplished using Equation 3.37 which is: 


$$
\gamma_{c}=\frac{\gamma_{h L} \gamma_{h H}(1-\operatorname{span})}{\left(\gamma_{h H} \operatorname{span}-\gamma_{h L}\right)\left(\gamma_{g}+1\right)}
$$

The results were plotted in Figure 5.2. The PS-CVT contains two reductions.

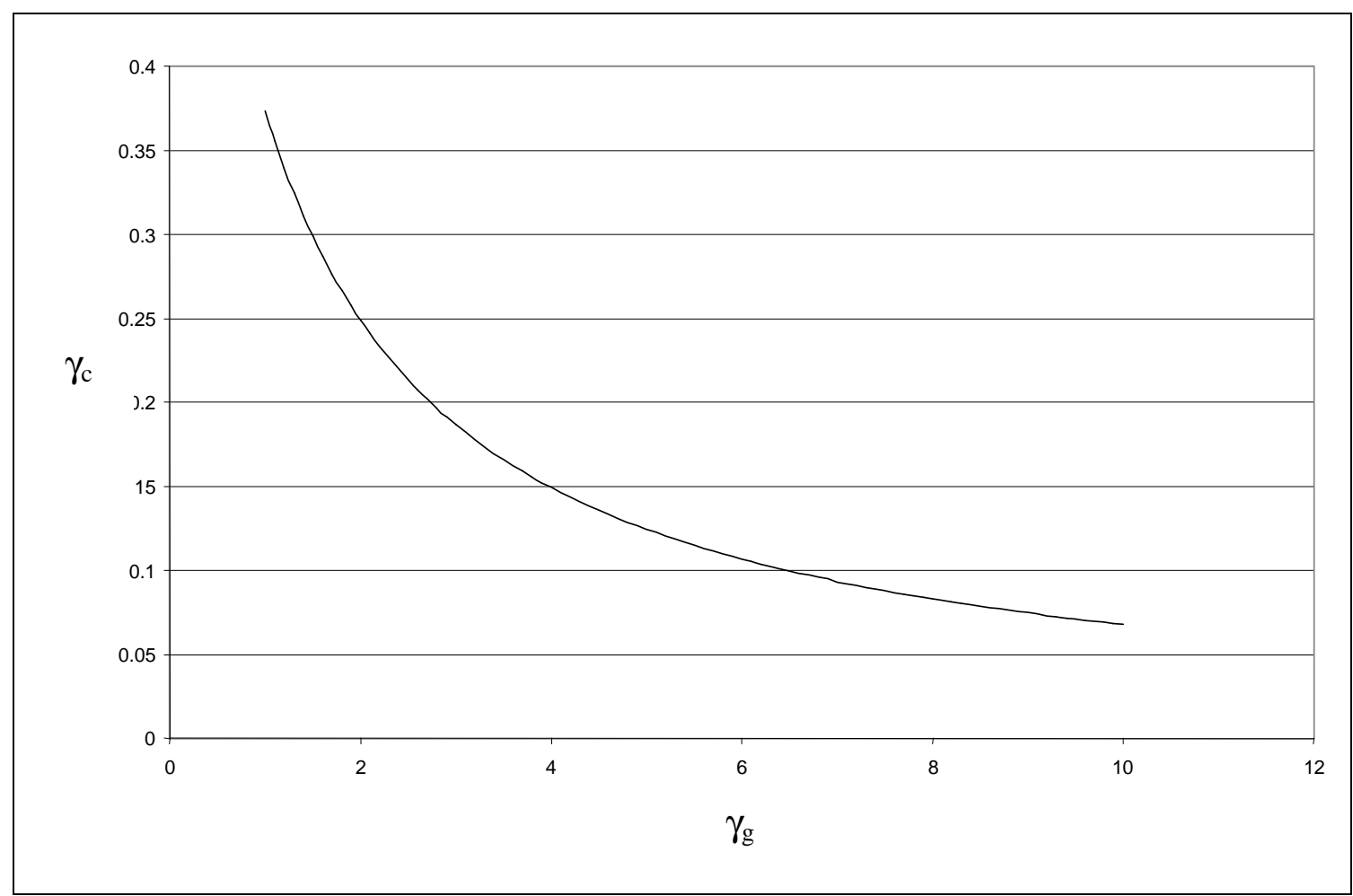

Figure 5.2: Possible combinations of $\gamma_{\mathrm{g}}$ and $\gamma_{\mathrm{c}}$ which yield a span of 1.52 for example application.

These include the power split unit, which is the input/output configuration and the gearbox, which contains the reductions for each stage. It is desirable that the resulting values of $\gamma_{\mathrm{g}}$ and $\gamma_{c}$ produce the smallest gear sizes for the two planetary gear trains of the power split unit. In Appendix A, $\gamma_{\mathrm{g}}$ and $\gamma_{\mathrm{c}}$ are defined as $\mathrm{N}_{\mathrm{R}} / \mathrm{N}_{\mathrm{S}}$ and $\mathrm{N}_{\mathrm{o}} / \mathrm{N}_{\mathrm{c}}$ respectively. Where $\mathrm{N}_{\mathrm{S}}$ and $\mathrm{N}_{\mathrm{o}}$ are the number of teeth on the sun gears and $\mathrm{N}_{\mathrm{R}}$ and $\mathrm{N}_{\mathrm{c}}$ are the number of teeth on the ring gears of these planetary gear trains. Since it is desirable to minimize gear size tooth counts for the sun gear is held at a fixed assumed, minimum value while $\mathrm{N}_{c}$ and $\mathrm{N}_{R}$ are allowed to vary. Figure 5.2 illustrates the inverse relationship between $\gamma_{\mathrm{g}}$ and $\gamma_{\mathrm{c}}$. Since $\mathrm{N}_{\mathrm{o}}$ and $\mathrm{N}_{\mathrm{S}}$ are held 
fixed. A decrease in $\gamma_{c}$ must be accomplished by an increase in $\mathrm{N}_{\mathrm{c}}$. Similarly, as $\gamma_{\mathrm{g}}$ increases, $N_{R}$ must be increased. Consequently moving left to right along the $\gamma_{\mathrm{g}}-\gamma_{c}$ curve results in increasing ring gear sizes. To reduce the size of these gear trains a $\gamma_{g}$ and $\gamma_{c}$ are selected from the left portion of the curve. The minimum value that can be used for $\gamma_{\mathrm{g}}$, considering geometric limitations, has been determined to be 1.5. The corresponding $\gamma_{c}$ is .3. These are the values used for the example application.

Step 9 of this procedure involves plotting a set of power balance curves using the input power and the efficiency of the coupling. The equations for the powers of configuration 3.2 were derived in chapter 3. The following Equation 3.36 was used to solve for $\mathrm{To}_{\mathrm{i}}$

$$
T o_{i}=\frac{T_{i n}}{1+\frac{\eta \gamma_{h}}{\gamma_{c}\left(\gamma_{g}+1\right)}}
$$

The value obtained was then substituted into the following equations (Equations 3.28 through 3.32) to obtain the powers of the system.

$$
\begin{gathered}
\mathrm{P}_{\text {sun }}=\mathrm{P}_{\text {in }}+\mathrm{Po}_{\mathrm{I}} \\
\mathrm{P}_{\text {cir }}=\mathrm{Po}_{\mathrm{i}}-\mathrm{P}_{\text {loss }} \\
\mathrm{P}_{\text {out }}=\mathrm{P}_{\text {sun }}+\mathrm{P}_{\text {cir }} \\
\mathrm{P}_{\text {loss }}=\mathrm{Po}_{\mathrm{i}}(1-\eta) \\
\mathrm{Po}_{\mathrm{i}}=\mathrm{To}_{\mathrm{i}} \omega_{\text {in }}
\end{gathered}
$$

The resulting powers are plotted against $\gamma_{\mathrm{h}}$ and is shown below in Figure 5.3. $\mathrm{Po}_{\mathrm{i}}$ is the amount of power that is transmitted through the coupling and has a maximum value of 180 hp as shown by the power balance curves. This is below the capacity of the selected coupling. 


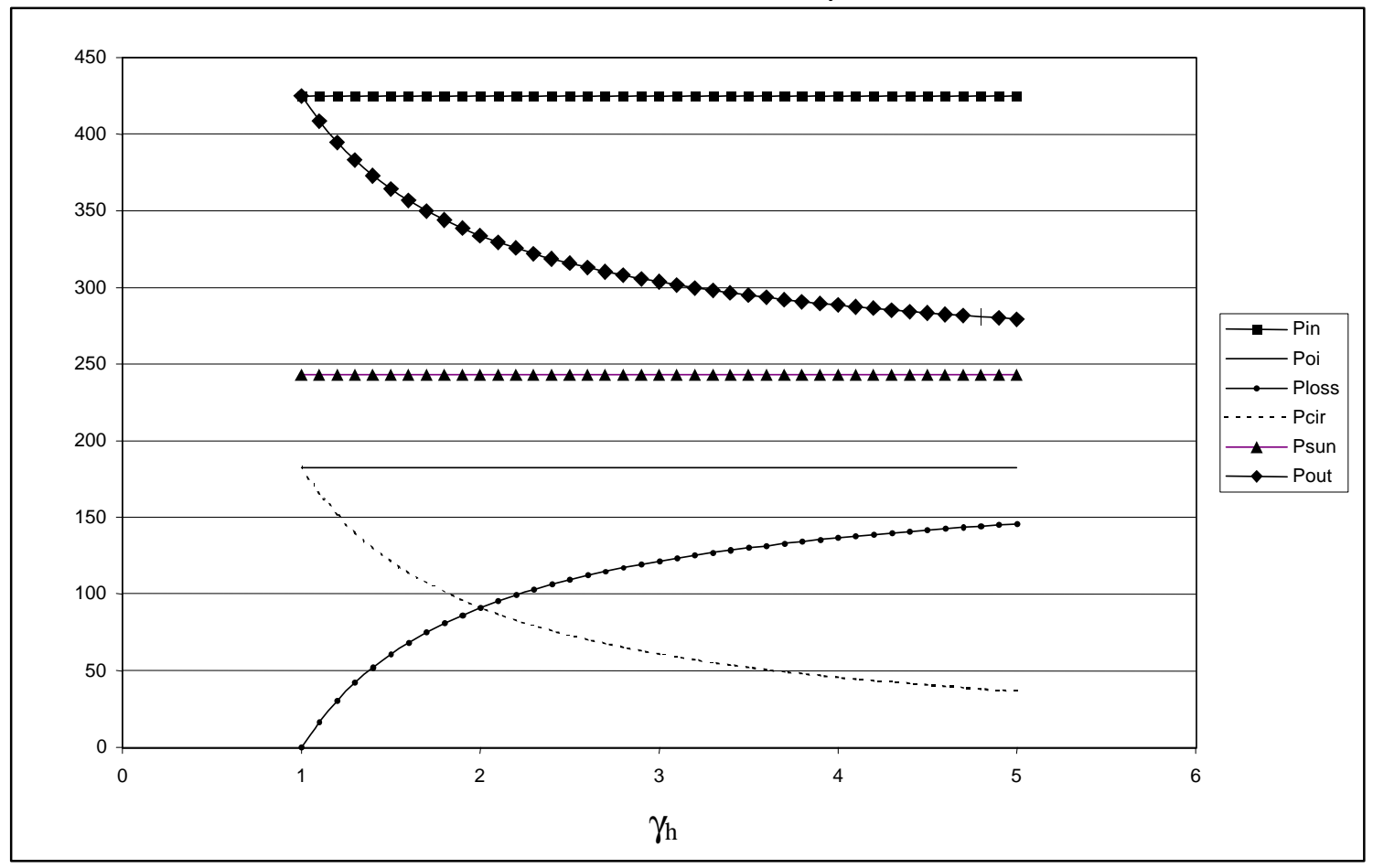

Figure 5.3: Power balance curves for example application.

In step 10 of the procedure the gearbox ratios, $\gamma_{1}, \gamma_{2}$, and $\gamma_{3}$ are determined. The power split unit reduction is fixed by the selection of $\gamma_{\mathrm{g}}$ and $\gamma_{\mathrm{c}}$. The low and high speed ratios of the power split unit were then calculated by substituting $\gamma_{\mathrm{hL}}$ and $\gamma_{\mathrm{hH}}$ for $\gamma_{\mathrm{h}}$ in the velocity ratio equation. For configuration 3.2, Equation 3.7,

$$
\frac{\omega_{\text {in }}}{\omega_{\text {out }}}=-\frac{\gamma_{g} \gamma_{h}}{\gamma_{h}+\gamma_{c}\left(\gamma_{g}+1\right)}
$$

was used to determine these values. In this application $\gamma_{\mathrm{hL}}$ and $\gamma_{\mathrm{hH}}$ are 5 and 1 respectively. Substitution of these values results in a low speed ratio of -1.3 and a high speed ratio -.85 . The gearbox ratios for each stage are calculated to meet the required ratios of the application. To determine $\gamma_{1}$, the lowest transmission ratio was divided by the low speed ratio of the power split unit. This yields a value for $\gamma_{1}$ of -1.55 . The high speed ratio of this stage was obtained by multiplying the high speed ratio of the power split unit by $\gamma_{1}$. The high speed 
ratio of stage one is 2.17 , for stepless shifts to occur, this must also be equal to the low speed ratio of the transmission for stage two. Calculation of $\gamma_{2}$ is accomplished by dividing this low speed ratio by the low speed ratio of the power split unit. This results in a value for $\gamma_{2}$ of -1.73. The high speed ratio of stage two was calculated using the same method as described for stage one. This process is repeated one more time in order to determine $\gamma_{3}$, which was -1.15. As a final check the high speed velocity ratio should be computed and compared to the overall required high speed velocity ratio. If the high speed ratio for the final stage was less then or equal to the overall required high speed ratio then the gearbox ratios have been determined correctly. For the example, the high speed ratio was .97, which compares favorably to the target value of 1.0.

Steps 12 through 15 calculate the gear sizes. A spreadsheet was set up to make this part of the procedure more efficient, since it requires several iterations. The resulting gear sizes for the example application can be found in Appendix B. The first step in sizing the gears was to select a diametral pitch. The initial selection of a diametral pitch for the planetary and control gears was 8 . For the gearbox gears a value of 5 was chosen. The initial number of teeth for each gear was selected to achieve the desired ratios calculated above. Gear selection was subject to the following two additional constraints. For the two planetary gear trains, the radius of the sun plus the diameter of the planet or idler should always equal to the radius of the ring gear. Since the gear ratios $\gamma_{1}, \gamma_{2}$, and $\gamma_{3}$ are on parallel shafts, the center distance between the gear sets should also be kept equal. The face width of each gear was calculated using AGMA bending strength standards (Avallone and Baumeister 1996, Shigley and Mischke 1989). The tangential load on each gear was calculated using the maximum powers transmitted by the gears, which was found from the power balance curves. 
By selecting a gear material and setting all the constants to standard values, the face width was calculated. The requirements for this application are to keep the ring gears of the planetary and control gear trains smaller than the diameter of the coupling and to keep the face width of all the gears less then 1.5 inches. After several iterations using the spreadsheet, satisfactory values were obtained. This completes the conceptual design procedure. The conceptual design is illustrated with two diagrams. Figure 5.4 is a wire-frame schematic diagram, which relates to Figure 5.5, the block diagram that describes the ratios and velocities of the transmission. 


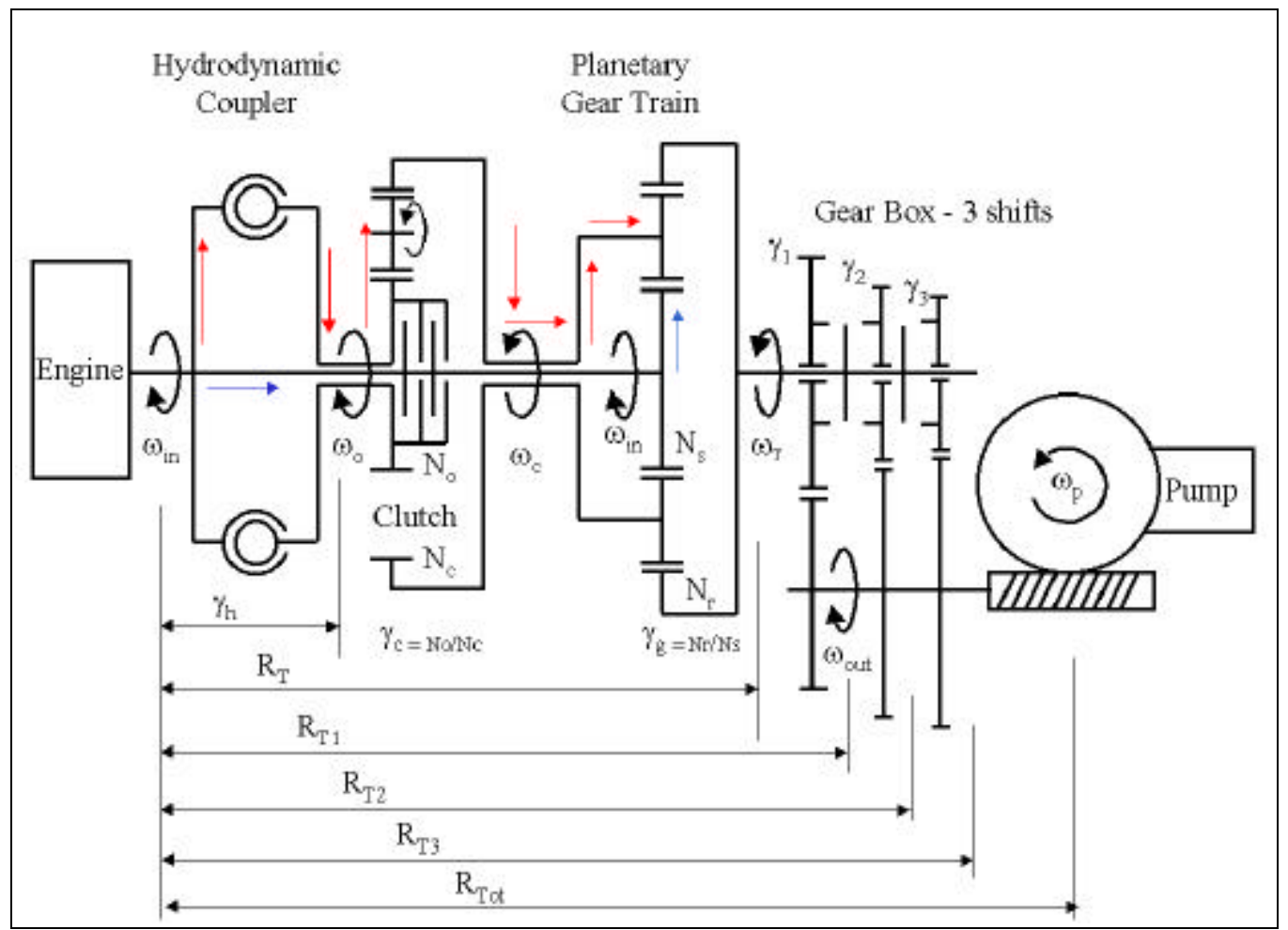

Figure 5.4: Description of example conceptual design system with coupling.

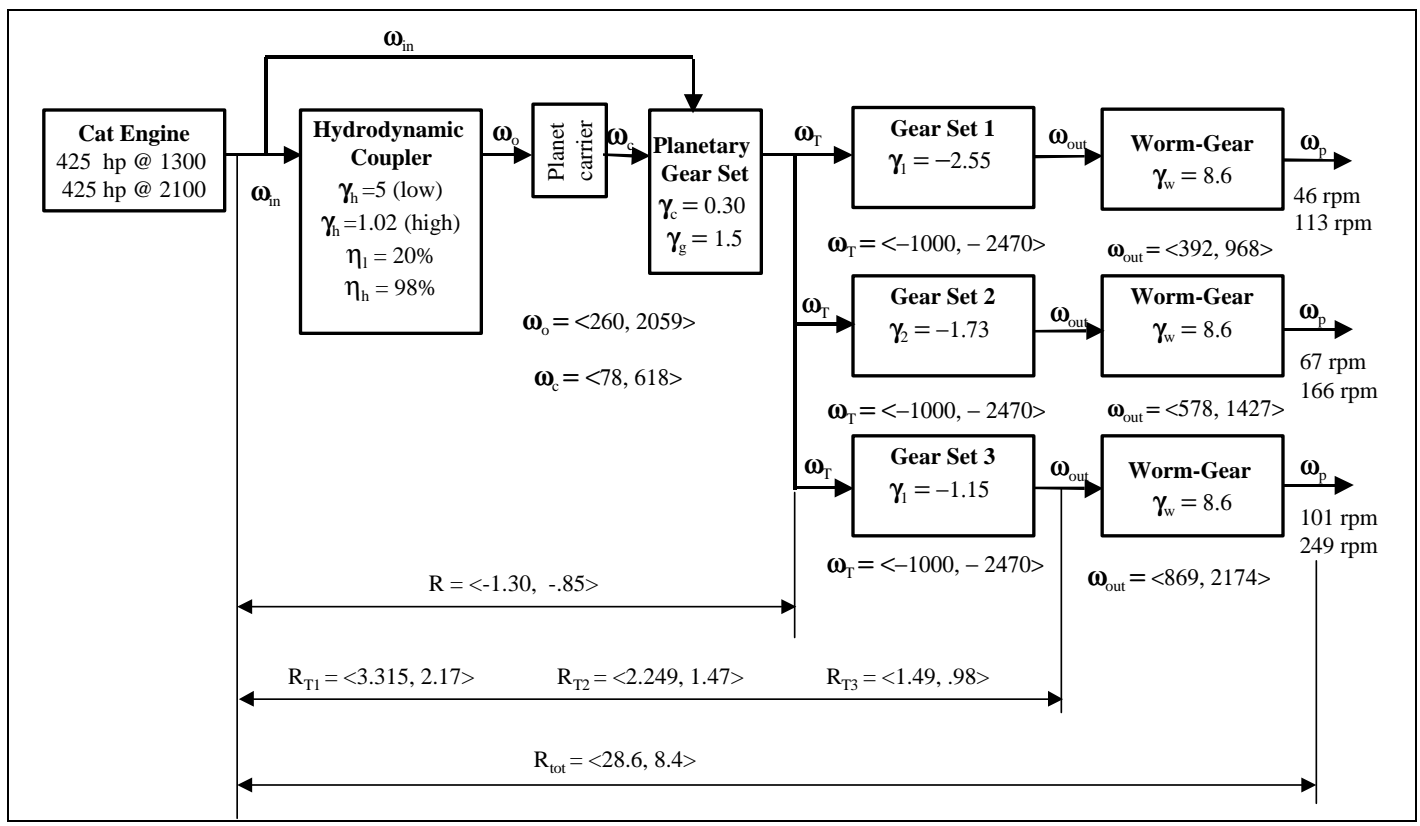

Figure 5.5: Block diagram of PS-CVT design using a Voith coupling for a well service pump.

Using the calculated values for gear ratios and sizes, and the selected coupling and output power, several things are determined about the design. First, the gear and coupling 
dimensions are used to develop a 2D drawing of the conceptual design. A 2D rendering of the conceptual PS-CVT design is presented in Figure 5.6. From this illustration in combination with the coupling gear sizes, the overall size of the transmission may be estimated. The overall sizes are then compared to those of the automatic transmission currently in use. These are presented in Table 5.1.

\begin{tabular}{|c|c|c|}
\hline Dimension & $\begin{array}{c}\text { Concept PS-CVT } \\
\text { w/ coupling }\end{array}$ & Automatic Transmission \\
\hline Length & $36 "$ & $41 "$ \\
\hline Width & $31.5 "$ & $21.8 "$ \\
\hline Height & $20 "$ & $26.6 "$ \\
\hline
\end{tabular}

Table 5.1: Dimension comparison of developed PS-CVT concept with automatic transmissions. 


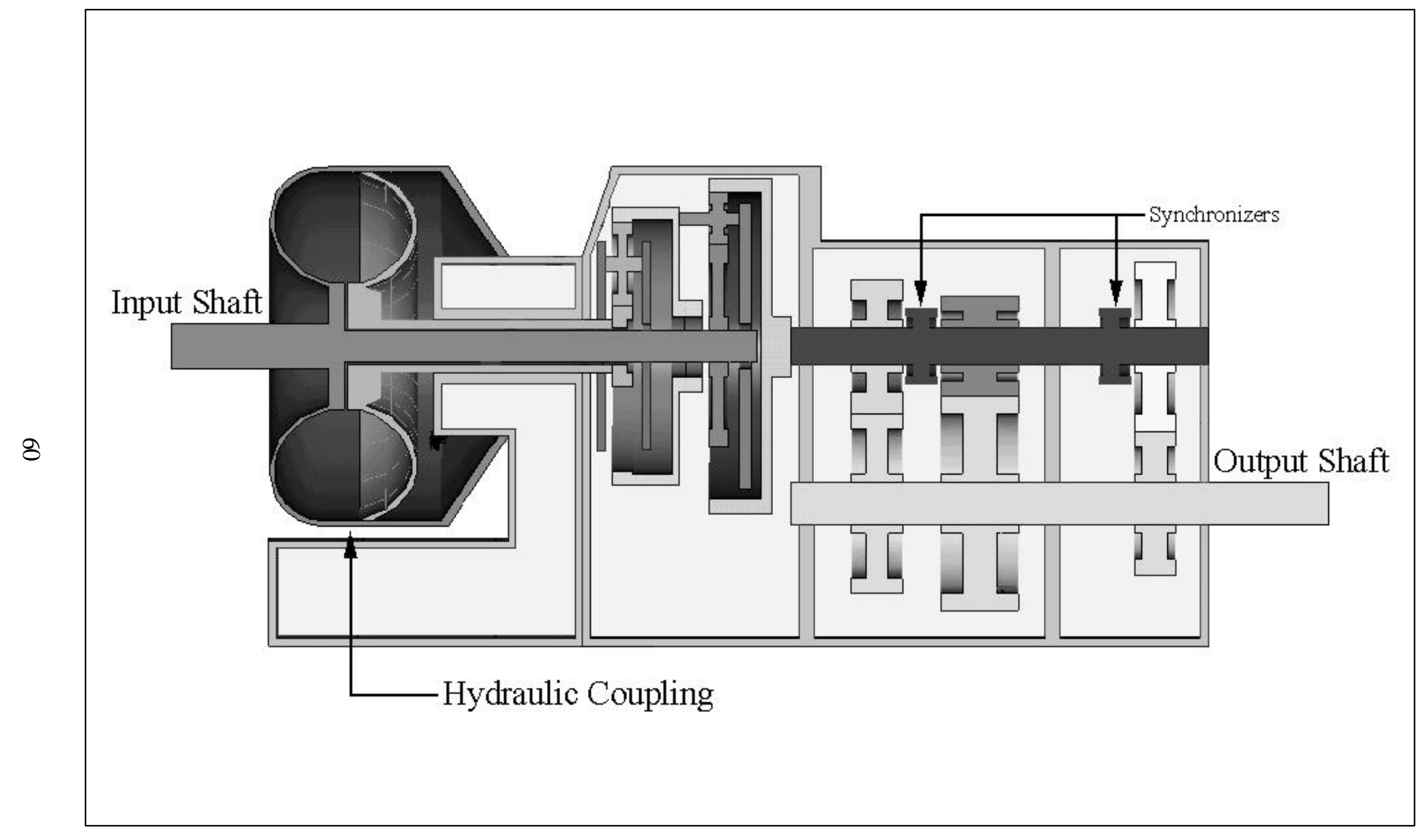

Figure 5.6: $2 \mathrm{D}$ rendering of the conceptual design using a coupling. 
The efficiency of this conceptual design was estimated using the power balance curves. Dividing the output power by the input power created Figure 5.7, of the estimated efficiencies.

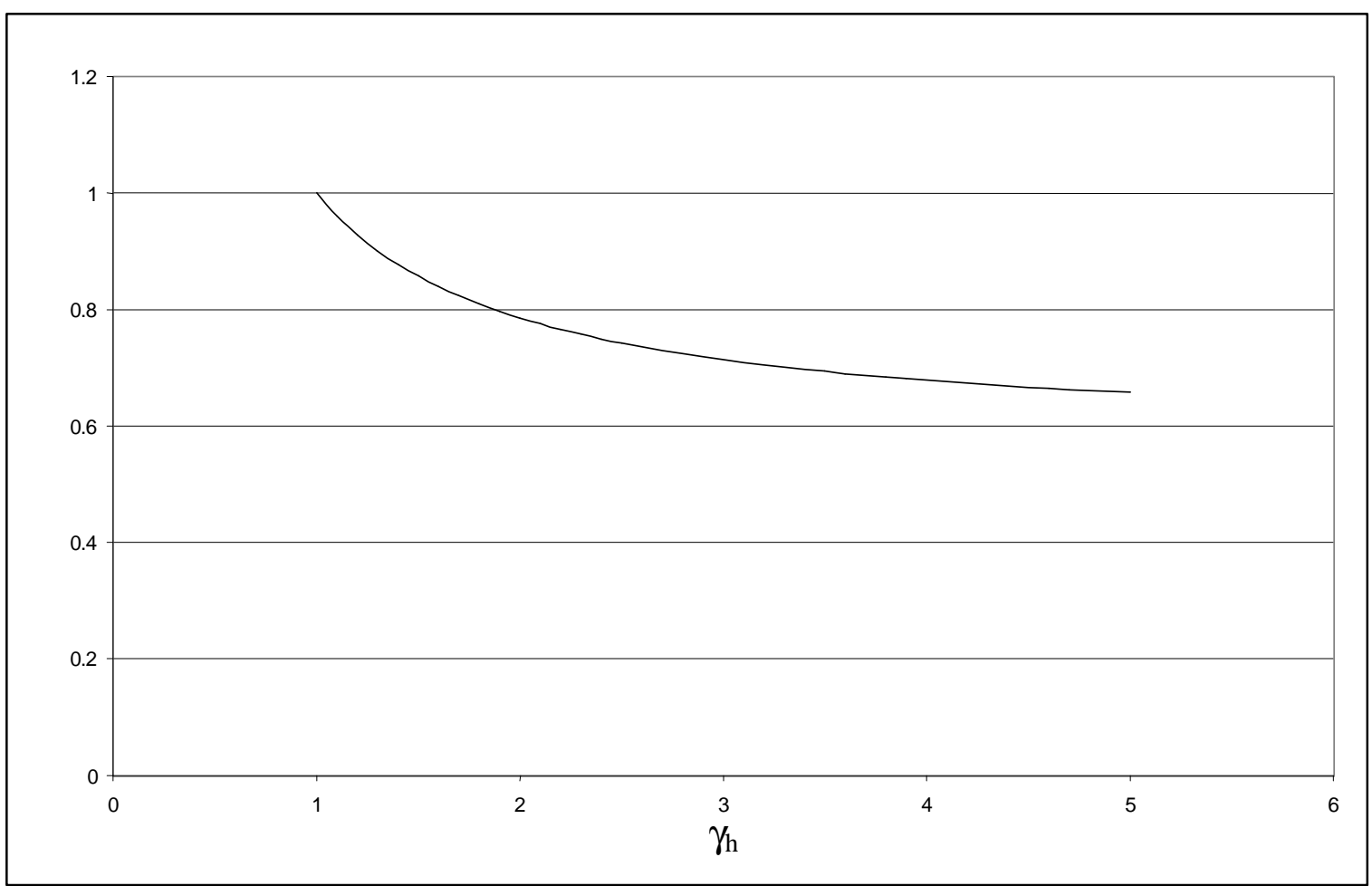

Figure 5.7: Estimated efficiency of the conceptual design using coupling.

\subsection{Conceptual design using a hydrostatic drive}

A second conceptual design for this application utilizes a hydrostatic drive for the variable element. Following the same steps outlined in the previous section the sizes and efficiencies of the alternative design are determined. The results of the process are presented in following figures. The overall design specifications are shown with a wire-frame diagram in Figure 5.8 and a block diagram shown in Figure 5.9. 


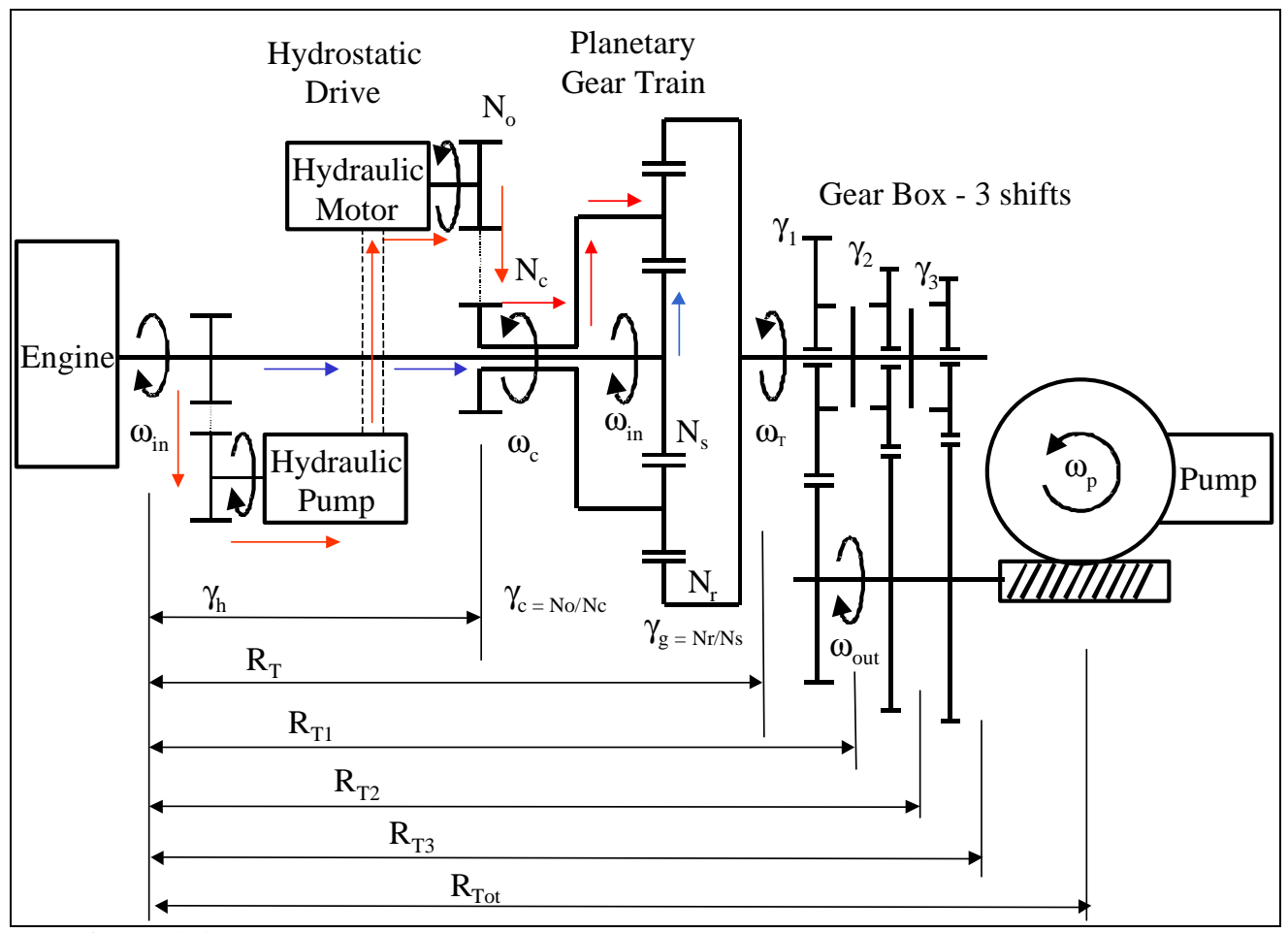

Figure 5.8: Description of example conceptual design system with hydrostatic drive.

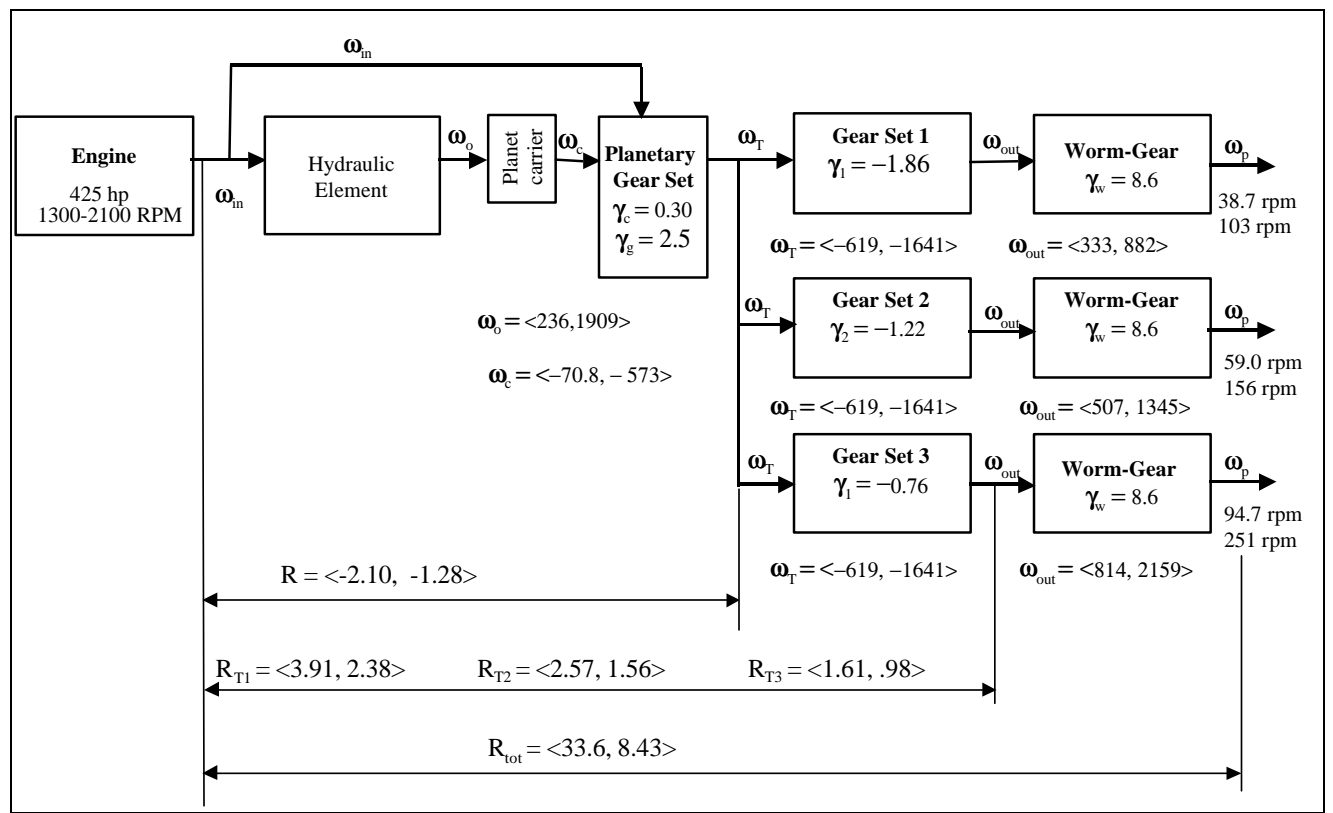

Figure 5.9: Block Diagram of PS-CVT design using a hydrostatic drive for a well service pump.

In the design procedure, the power balance diagram, Figure 5.10, was developed for the hydrostatic drive. The estimated efficiency was plotted in Figure 5.11 using the data from the power balance curve. 


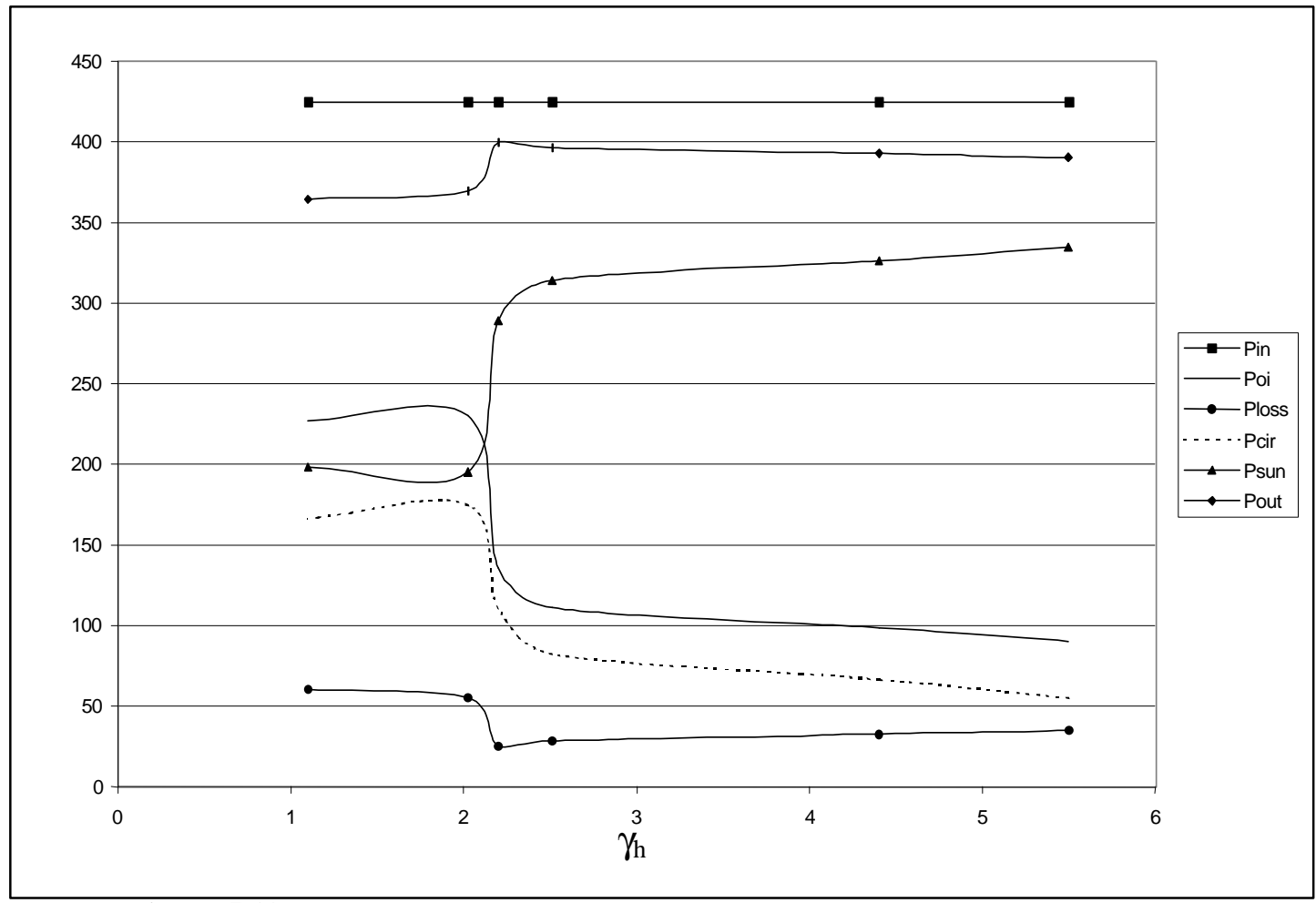

Figure 5.10: Power balance curve for conceptual PS-CVT using a hydrostatic drive.

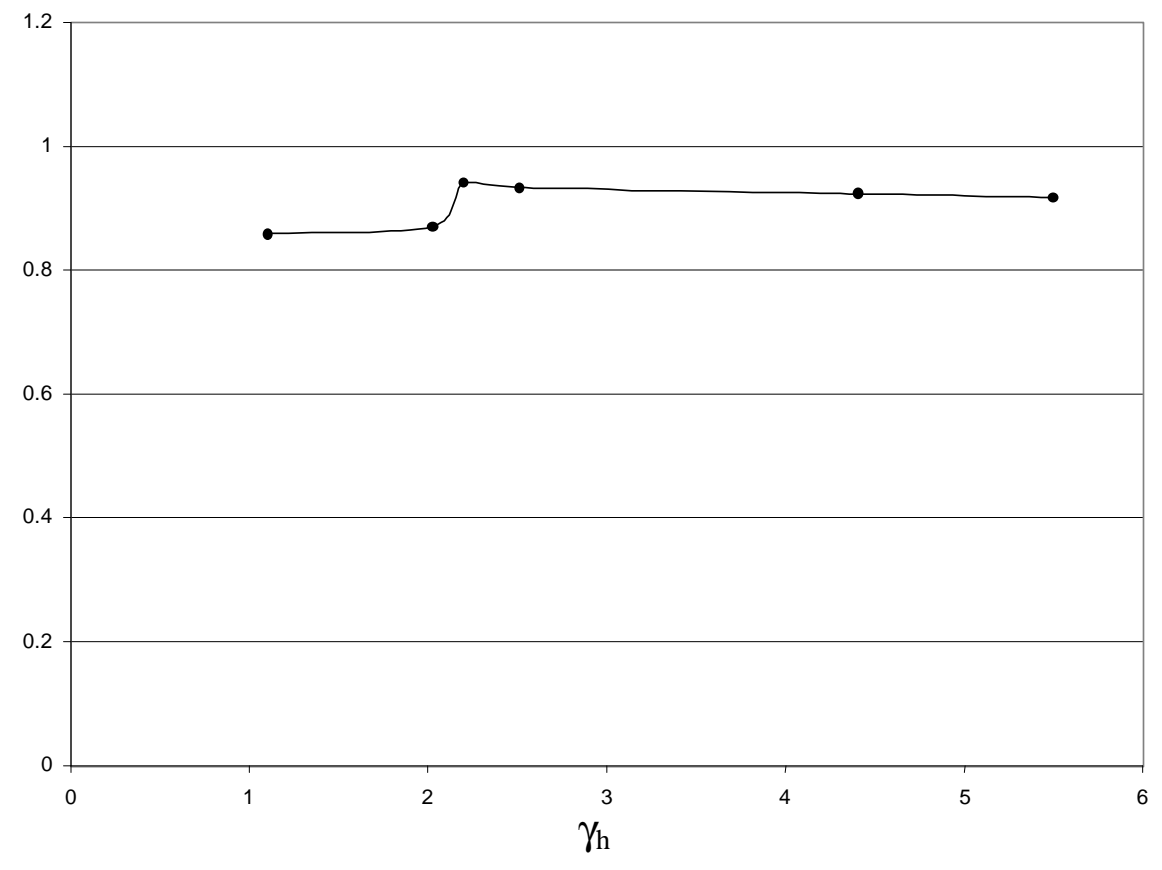

Figure 5.11: Estimated efficiency of the PS-CVT with hydrostatic drive. 
With the results for the procedure a $2 \mathrm{D}$ drawing of the conceptual design is presented in Figure 5.12. Using this illustration, along with the gear sizes (found in Appendix C) the overall dimensions for this design were estimated. The length, width, and height is 25 ", 15 ", and $26 "$ respectively.

Close examination of the results of the two conceptual designs reveals that the size and efficiency of the design utilizing a hydrostatic drive is more favorable than those of the hydrodynamic coupling configuration. The examples illustrated that the conceptual design procedure can be utilized to determine if a PS-CVT is a feasible replacement option for a given application. They also show that the information resulting from the conceptual design procedure facilitates the comparison of alternative PS-CVT designs. The use of a standardized design procedure allows for progression through the conceptual design phase of the application in an effective manner. 


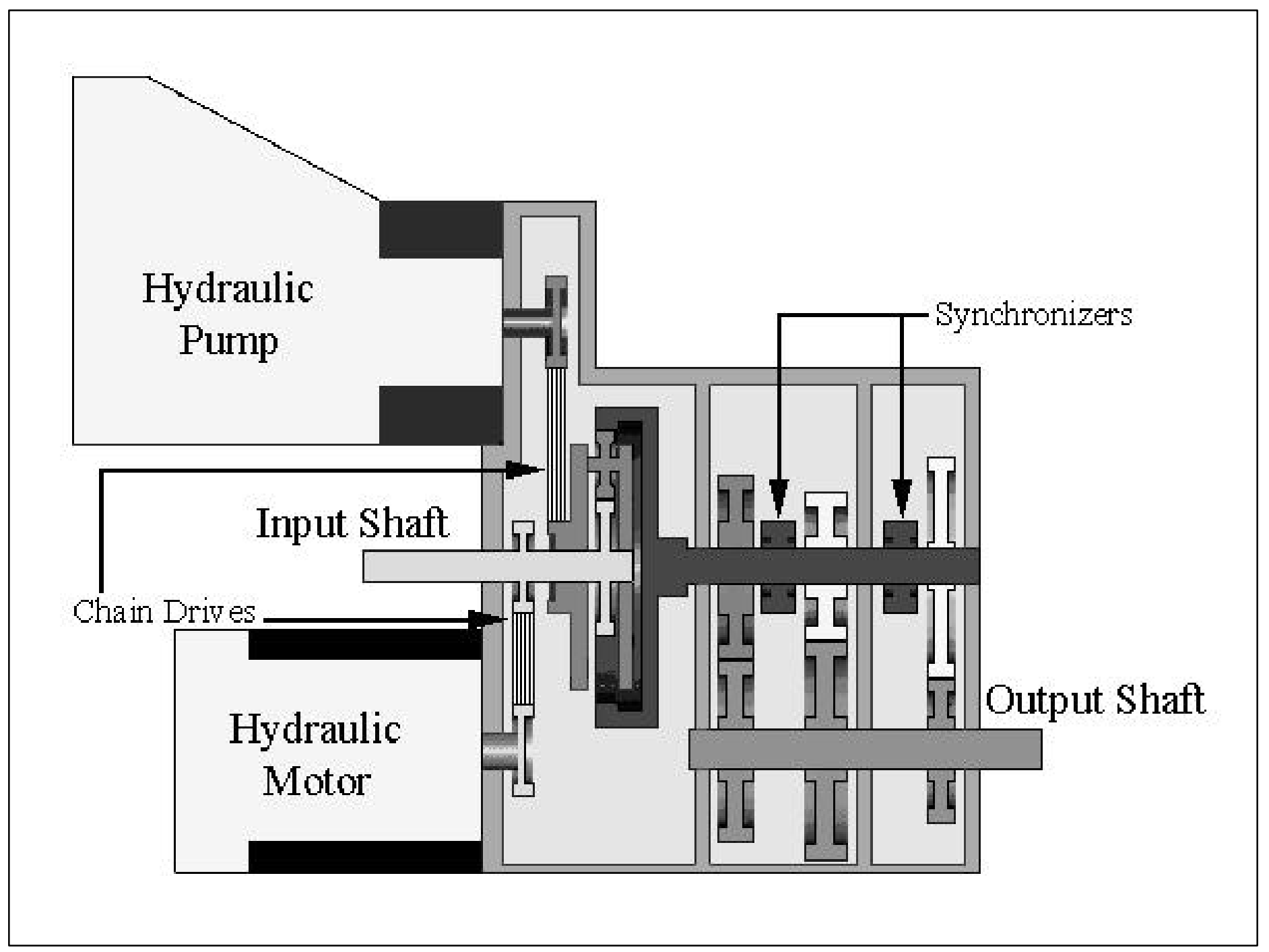

Figure 5.12: 2D illustration of the PS-CVT utilizing a hydrostatic drive. 


\section{Chapter 6 Conclusions}

\subsection{Conclusions}

Although much research has been devoted to the development of continuously variable transmissions, a literature search returned limited information on conceptual design procedures for a PS-CVT. The majority of the research to date has dealt with specialized designs that consist of a configuration and a variable element, for a specific application.

The purpose of this work was to develop a conceptual design procedure for a power split continuously variable transmission for industrial applications. This 15 step procedure was outlined using a flow chart. The results of the procedure can be used to estimate overall size, produce 2D and 3D scale drawings and estimate efficiencies of the conceptual transmission. This procedure offers a means to improve the efficiency of the conceptual design stage of a PS-CVT. It also allows the designer to determine more effectively the feasibility of a PS-CVT for a specific application in the conceptual stages.

A industrial pump was used as an example industrial application to illustrate the conceptual design procedure. Two conceptual designs, using different variable elements where completed for this application. The two variable elements considered were a hydrodynamic coupling and a hydrostatic drive. The two examples also illustrated how the results of the procedure can be used to determine whether a PS-CVT is a viable alternative for the well service pump system. 


\subsection{Contributions}

The work of this thesis has several contributions to the knowledge of power split continuously variable transmission design as well as contributions to Halliburton Energy Services. The configuration charts can be used to streamline the process of determining an input coupled variable shunt configuration for a given application. The conceptual design procedure can be used to: develop a PS-CVT, compare conceptual designs, and determine feasibility of PS-CVTs for specific applications. In the process of demonstrating the procedure with examples of an industrial pumping application, the feasibility of a PS-CVT for an industrial pump application was studied.

\subsection{Recommendations}

It is recommended that further refinement of the conceptual procedure be completed. This would include optimization of several steps, such as gear sizes, gearbox ratios, and the span. Several steps may also be added. These would include determining shaft sizes, bearing sizes and weights, and housings material and size for the PS-CVT. Considering the number of combinations and steps involved, it would be useful to develop a conceptual design program. This could make the conceptual design stage of a PS-CVT even more efficient. 


\section{Bibliography}

Avallone, Eugene A., and Theodore Baumeister III. Marks' Standard Handbook for Mechanical Engineers Tenth Edition. New York: McGraw-Hill, 1996.

Beachley, N. H., et al. "Evaluation of Split-path Extended Range Continuously-variable Transmissions for Automotive Applications," Journal of the Franklin Institute Vol. 317, No. 4 (1984 ): 235-262.

Bretz, Lyle, Team Leader of Marketing Technical Services. Telephone interview at SauerSundstrand Company, Ames, Iowa. from Morgantown, WV., 12 November 1998.

Cowen, Ben. “Variable Speed Transmissions,” U.S. Patent No. 5,167,591, Dec. 1, 1992

Cowen, Ben. "Variable Speed Transmissions," U.S. Patent No. 5,215,323, Jun. 1, 1993

Dorey, R. E., and N. D. Vaughan. "Computer Aided Design of Split Power Hydrostatic Transmission Systems," CAD in High Pressure Hydraulics. London, England: ImechE, 1983.61-69

Hausenbauer, Tom C. Design Study of a Power Split Hydrostatic Transmission. Masters Thesis, University of Wisconsin-Madison, 1976.

Hsieh, Long-Chang, and Hong-Sen Yan. "On the Mechanical Efficiency of Continuously Variable Transmissions with Planetary Gear Trains." International Journal of Vehicle Design 11.2 (1990): 176-187.

Kluger, Michael A., and Douglas R. Fussner. "An Overview of Current CVT Mechanisms, Forces and Efficiencies," SAE Technical Paper No. 970688, 1997.

Kumm, Emerson L. "Continuously Variable Transmission Using Planetary Gearing with Regenerative Torque Transfer and Employing Belt Slip to Measure and Control Pulley Torque," U.S. Patent No. 5,011,458, Apr. 30, 1991.

Lemmens, John. “Variable Speed Transmission,” U.S. Patent No. 3,641,843, Feb. 15, 1972.

Lu, Zhijian, Acceleration Simulation of a Vehicle with a Continuously Variable Power Split Transmission. Masters Thesis, West Virginia University, 1998.

Lu, Zhijian, et al. "Simulation of a Continously Variable Power Split Transmission," SAE Technical Paper No. 1999-01-0062, 1999.

Mabie, Hamilton H., and Charles F. Reinholtz. Mechanisms and Dynamics of Machinery. New York: John Wiley \& Sons, 1987. 
Mucino, Victor H., et al. "A Double Planetary Gear Train-CVT Transmission with Multiple Applications," SAE Technical Paper No. 950094, 1995.

Orshansky, Eli, and William E. Weseloh. "Characteristics of Multiple Range Hydromechanical Transmissions," SAE Technical Paper No. 720724, 1972.

Sauer-Sundstrand Company. Axial Piston Pumps (Series 90) Technical Information. Rev. C. May 1996.

Sauer-Sundstrand Company. Bent Axis Variable Motors (Series 51) Technical Information. Rev. C. March 1997.

Sheu, K. B, et al. "Conceptual Design of Hybrid Transmissions for Motorcycle applications." International Journal of Vehicle Design, Vol. 17, No. 4, (1996): 430448

Shigley, Joseph E., and Charles R. Mischke. Mechanical Engineering Design Fifth Edition. New York: McGraw-Hill, 1989.

Takayama et al. "Continuously Variable Transmission," U.S. Patent No. 5,050,457, Sep. 24, 1991.

Voith Transmission Inc. Variable-Speed Turbo Couplings. September 1997.

White, G. "Compounded Two-Path Variable Ratio Transmissions with Coaxial Gear Trains." Mechanism and Machine Theory, Vol. 11 (1976): 227-240.

White, G. "A Two-Path Variable-Ratio Transmission with an Extended Range of Ratios," ASME Paper No. 76-DET-37, 1976. 


\section{Appendix A: Configuration Charts}




\begin{tabular}{|c|c|c|c|c|c|c|c|c|}
\hline Config & input variables & $\omega_{c}$ & output variables & $\omega_{\text {out }}$ & $\omega_{r} / \omega_{\text {aut }}$ & Power split & Curves & \\
\hline 1.1 & $\begin{array}{l}\gamma_{h}=\frac{\Phi_{i n}}{\omega_{o}} \\
\gamma_{c}=\frac{N_{o}}{N_{c}} \\
\gamma_{n}=\frac{N_{2}}{N_{1}} \\
N_{o}+N_{1}=N_{c}+N_{2}\end{array}$ & $\varpi_{i n} \frac{\gamma_{c} \gamma_{n}}{\gamma_{h}}$ & $\begin{array}{l}\gamma_{g}=\frac{N_{R}}{N_{s}} \\
\varpi_{s}=\bar{\varpi}_{\text {in }} \\
\bar{\varpi}_{a}=\varpi_{\text {out }} \\
\varpi_{R}=\bar{\varpi}_{c}\end{array}$ & $\frac{\bar{\varpi}_{i n}+\gamma_{g} \bar{\varpi}_{c}}{\gamma_{g}+1}$ & $\frac{\gamma_{g} \gamma_{h}+\gamma_{h}}{\gamma_{h}+\gamma_{c} \gamma_{n} \gamma_{g}}$ & $\begin{array}{c}R=\frac{\gamma_{g} \gamma_{h}+\gamma_{h}}{\gamma_{h}+\gamma_{c} \gamma_{h} \gamma_{s}} \\
\quad r=\frac{\gamma_{c} \gamma_{n}}{\gamma_{h}} \\
\gamma=\frac{r(1-R)}{1-r}\end{array}$ & 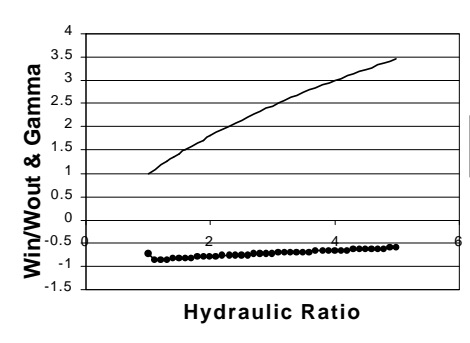 & 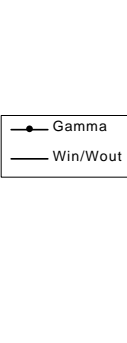 \\
\hline 1.2 & $\begin{array}{l}\gamma_{h}=\frac{\sigma_{i n}}{\omega_{o}} \\
\gamma_{c}=\frac{N_{o}}{N_{c}} \\
\gamma_{n}=\frac{N_{2}}{N_{1}} \\
N_{o}+N_{1}=N_{c}+N_{2}\end{array}$ & $\varpi_{i n} \frac{\gamma_{c} \gamma_{n}}{\gamma_{h}}$ & $\begin{array}{l}\gamma_{g}=\frac{N_{R}}{N_{s}} \\
\varpi_{s}=\varpi_{\text {in }} \\
\varpi_{a}=\varpi_{c} \\
\varpi_{R}=\varpi_{\text {out }}\end{array}$ & $\frac{\Phi_{c}\left(\gamma_{g}+1\right)-\Phi_{i n}}{\gamma_{g}}$ & $-\frac{\gamma_{g} \gamma_{h}}{\gamma_{h}-\gamma_{c} \gamma_{n}\left(\gamma_{g}+1\right)}$ & $\begin{array}{c}R=\frac{\gamma_{g} \gamma_{h}}{\left.\gamma_{h}-\gamma_{C} \gamma_{n}+1\right)} \\
r=\frac{\gamma_{c} \gamma_{n}}{\gamma_{h}} \\
\gamma=\frac{r(1-R)}{1-r}\end{array}$ & Hydraulic Ratio & 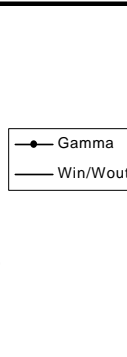 \\
\hline 1.3 & $\begin{array}{l}\gamma_{h}=\frac{\Phi_{i n}}{\Phi_{o}} \\
\gamma_{c}=\frac{N_{o}}{N_{c}} \\
\gamma_{n}=\frac{N_{2}}{N_{1}} \\
N_{o}+N_{1}=N_{c}+N_{2}\end{array}$ & $\varpi_{i n} \frac{\gamma_{c} \gamma_{n}}{\gamma_{h}}$ & $\begin{array}{l}\gamma_{g}=\frac{N_{R}}{N_{s}} \\
\varpi_{s}=\varpi_{o u t} \\
\varpi_{a}=\varpi_{\text {in }} \\
\varpi_{R}=\varpi_{c}\end{array}$ & $\Phi_{i n}\left(\gamma_{g}+1\right)-\gamma_{g} \bar{\omega}_{c}$ & $\frac{\gamma_{h}}{\gamma_{h}\left(\gamma_{g}+1\right)-\gamma_{c} \gamma_{n} \gamma_{g}}$ & $\begin{array}{c}R=\frac{\gamma_{h}}{\gamma_{h}\left(\gamma_{g}+1\right)-\gamma_{c h} \gamma_{g}} \\
r=\frac{\gamma_{c} \gamma_{n}}{\gamma_{h}} \\
\gamma=\frac{r(1-R)}{1-r}\end{array}$ & 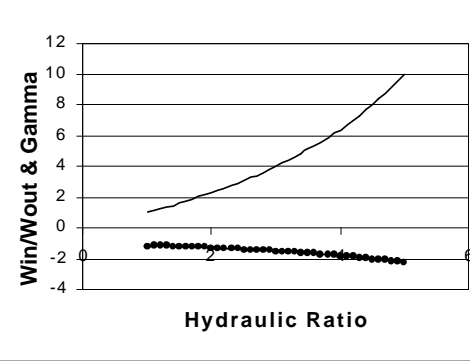 & 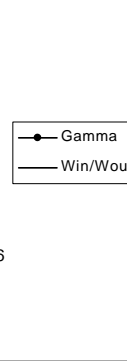 \\
\hline 1.4 & $\begin{array}{l}\gamma_{h}=\frac{\Phi_{i n}}{\Phi_{o}} \\
\gamma_{c}=\frac{N_{o}}{N_{c}} \\
\gamma_{n}=\frac{N_{2}}{N_{1}} \\
N_{o}+N_{1}=N_{c}+N_{2}\end{array}$ & $\varpi_{i n} \frac{\gamma_{c} \gamma_{n}}{\gamma_{h}}$ & $\begin{array}{l}\gamma_{g}=\frac{N_{R}}{N_{s}} \\
\bar{\varpi}_{s}=\bar{\varpi}_{c} \\
\bar{\varpi}_{a}=\bar{\varpi}_{\text {in }} \\
\bar{\varpi}_{R}=\bar{\varpi}_{\text {out }}\end{array}$ & $\frac{\Phi_{i n}\left(\gamma_{g}+1\right)-\Phi_{c}}{\gamma_{g}+1}$ & $\frac{\gamma_{h}\left(\gamma_{g}+1\right)}{\gamma_{h}\left(\gamma_{g}+1\right)-\gamma_{c} \gamma_{n}}$ & $\begin{array}{c}R=\frac{\gamma_{h}\left(\gamma_{g}+1\right)}{\gamma_{h}\left(\gamma_{g}+1\right)-\gamma_{c} \gamma_{n}} \\
r=\frac{\gamma_{c} \gamma_{n}}{\gamma_{h}} \\
\gamma=\frac{r(1-R)}{1-r}\end{array}$ & 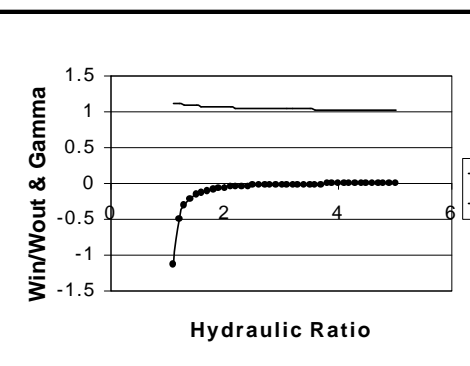 & 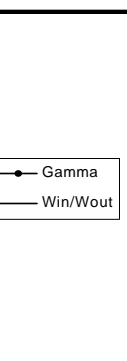 \\
\hline
\end{tabular}




\begin{tabular}{|c|c|c|c|c|c|c|c|c|}
\hline Sonfig. & input variables & $\omega_{c s}$ & output variables & $\omega_{\text {out }}$ & $\omega_{n} / \omega_{\text {out }}$ & Power split & Curves & \\
\hline 1.5 & $\begin{array}{l}\gamma_{h}=\frac{\bar{\omega}_{i n}}{\Phi_{o}} \\
\gamma_{c}=\frac{N_{o}}{N_{c}} \\
\gamma_{n}=\frac{N_{2}}{N_{1}} \\
N_{o}+N_{1}=N_{c}+N_{2}\end{array}$ & $\varpi_{i n} \frac{\gamma_{c} \gamma_{n}}{\gamma_{h}}$ & $\begin{array}{l}\gamma_{g}=\frac{N_{R}}{N_{s}} \\
\varpi_{s}=\bar{\varpi}_{c} \\
\bar{\varpi}_{a}=\bar{\varpi}_{\text {out }} \\
\bar{\varpi}_{R}=\bar{\varpi}_{\text {in }}\end{array}$ & $\frac{\overline{\boldsymbol{\Phi}}_{c}+\gamma_{g} \overline{\boldsymbol{\omega}}_{i n}}{\gamma_{g}+1}$ & $\frac{\gamma_{h}\left(\gamma_{g}+1\right)}{\gamma_{h} \gamma_{g}+\gamma_{c} \gamma_{n}}$ & $\begin{array}{c}R=\frac{\gamma_{h}\left(\gamma_{g}+1\right)}{\gamma_{h} \gamma_{g}+\gamma_{c} \gamma_{n}} \\
\quad r=\frac{\gamma_{c} \gamma_{n}}{\gamma_{h}} \\
\gamma=\frac{r(1-R)}{1-r}\end{array}$ & 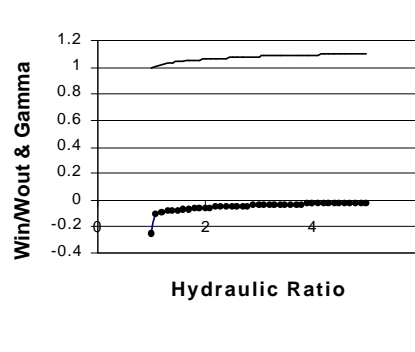 & 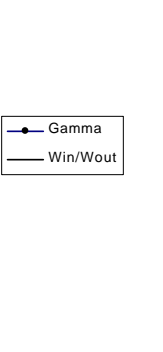 \\
\hline 1.6 & $\begin{array}{l}\gamma_{h}=\frac{\sigma_{\text {in }}}{\omega_{o}} \\
\gamma_{c}=\frac{N_{o}}{N_{c}} \\
\gamma_{n}=\frac{N_{2}}{N_{1}} \\
N_{o}+N_{1}=N_{c}+N_{2}\end{array}$ & $\bar{\varpi}_{i n} \frac{\gamma_{c} \gamma_{n}}{\gamma_{h}}$ & $\begin{array}{l}\gamma_{g}=\frac{N_{R}}{N_{s}} \\
\varpi_{s}=\varpi_{\text {out }} \\
\varpi_{a}=\varpi_{c} \\
\varpi_{R}=\varpi_{\text {in }}\end{array}$ & $\gamma_{g}\left(\bar{\omega}_{c}-\bar{\omega}_{i n}\right)+\Phi_{c}$ & $-\frac{\gamma_{h}}{\gamma_{g} \gamma_{h}-\gamma_{c} \gamma_{n}\left(\gamma_{g}+1\right)}$ & $\begin{array}{c}R=-\frac{\gamma_{h}}{\gamma_{s} \gamma_{h}-\gamma_{c h}\left(\gamma_{s}+1\right)} \\
r=\frac{\gamma_{c} \gamma_{n}}{\gamma_{h}} \\
\gamma=\frac{r(1-R)}{1-r}\end{array}$ & 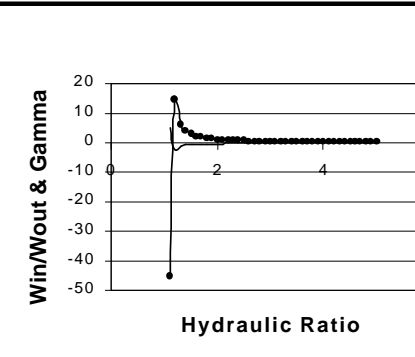 & $\begin{array}{l}\overrightarrow{-}_{- \text {Gamma }}^{\text {Girnout }} \\
\text { - }\end{array}$ \\
\hline 2.1 & $\begin{array}{l}\gamma_{h}=\frac{\sigma_{i n}}{\omega_{o}} \\
\gamma_{c}=\frac{N_{o}}{N_{c}} \\
\gamma_{n}=\frac{N_{2}}{N_{1}} \\
N_{o}+N_{1}=N_{c}+N_{2}\end{array}$ & $-\sigma_{i n} \frac{\gamma_{c} \gamma_{n}}{\gamma_{n}}$ & $\begin{array}{l}\gamma_{g}=\frac{N_{R}}{N_{s}} \\
\varpi_{s}=\bar{\varpi}_{\text {in }} \\
\varpi_{a}=\varpi_{\text {out }} \\
\varpi_{R}=\varpi_{c}\end{array}$ & $\frac{\bar{\varpi}_{i n}+\gamma_{g} \bar{\varpi}_{c}}{\gamma_{g}+1}$ & $\frac{\gamma_{g} \gamma_{h}+\gamma_{h}}{\gamma_{h}-\gamma_{c} \gamma_{n} \gamma_{g}}$ & $\begin{array}{c}R=\frac{\gamma_{g} \gamma_{h}+\gamma_{h}}{\gamma_{h}-\gamma_{c} \gamma_{n} \gamma_{g}} \\
r=\frac{\gamma_{c} \gamma_{n}}{\gamma_{h}} \\
\gamma=\frac{r(1-R)}{1-r}\end{array}$ & 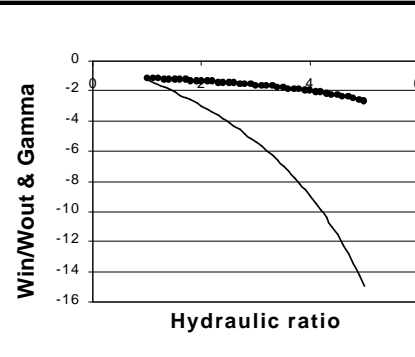 & 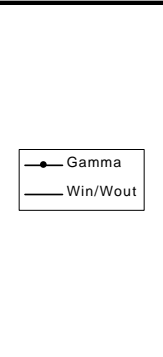 \\
\hline 2.2 & $\begin{array}{l}\gamma_{h}=\frac{\sigma_{i n}}{\bar{\omega}_{o}} \\
\gamma_{c}=\frac{N_{o}}{N_{c}} \\
\gamma_{n}=\frac{N_{2}}{N_{1}} \\
N_{o}+N_{1}=N_{c}+N_{2}\end{array}$ & $-\omega_{i n} \frac{\gamma_{c} \gamma_{n}}{\gamma_{n}}$ & $\begin{array}{l}\gamma_{g}=\frac{N_{R}}{N_{s}} \\
\varpi_{s}=\bar{\varpi}_{\text {in }} \\
\varpi_{a}=\varpi_{c} \\
\varpi_{R}=\varpi_{\text {out }}\end{array}$ & $\frac{\Phi_{c}\left(\gamma_{g}+1\right)-\bar{\varpi}_{i n}}{\gamma_{g}}$ & $\frac{\gamma_{g} \gamma_{h}}{\gamma_{h}+\gamma_{c} \gamma_{n}\left(\gamma_{g}+1\right)}$ & $\begin{array}{c}R=-\frac{\gamma_{8} \gamma_{h}}{\gamma_{h}+\gamma_{c} \gamma_{n}\left(\gamma_{8}+1\right)} \\
r=\frac{\gamma_{c} \gamma_{n}}{\gamma_{h}} \\
\gamma=\frac{r(1-R)}{1-r}\end{array}$ & 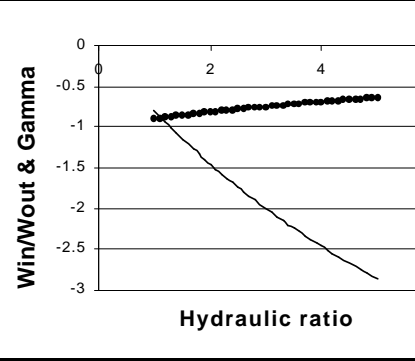 & 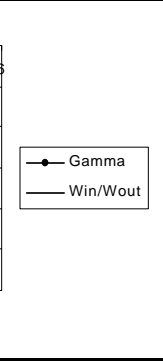 \\
\hline
\end{tabular}




\begin{tabular}{|c|c|c|c|c|c|c|c|c|}
\hline jonfig & variables & $\omega_{s}$ & output variables & $\omega_{\text {out }}$ & $\omega_{n} / \omega_{\text {out }}$ & Power split & Curves & \\
\hline 2.3 & $\begin{array}{l}\gamma_{h}=\frac{\sigma_{n_{n}}}{\bar{\omega}_{o}} \\
\gamma_{c}=\frac{N_{o}}{N_{c}} \\
\gamma_{n}=\frac{N_{2}}{N_{1}} \\
N_{o}+N_{1}=N_{c}+N_{2}\end{array}$ & $-\Phi_{i n} \frac{\gamma_{c} \gamma_{n}}{\gamma_{n}}$ & $\begin{array}{l}\gamma_{g}=\frac{N_{R}}{N_{s}} \\
\varpi_{s}=\bar{\varpi}_{\text {out }} \\
\varpi_{a}=\bar{\varpi}_{\text {in }} \\
\varpi_{R}=\varpi_{c}\end{array}$ & $\varpi_{i n}\left(\gamma_{g}+1\right)-\gamma_{g} \bar{\omega}_{c}$ & $\frac{\gamma_{h}}{\gamma_{h}\left(\gamma_{g}+1\right)+\gamma_{c} \gamma_{n} \gamma_{g}}$ & $\begin{array}{c}R=\frac{\gamma_{h}}{\gamma_{h}\left(\gamma_{g}+1\right)+\gamma \gamma_{h} \gamma_{g}} \\
r=\frac{\gamma_{c} \gamma_{n}}{\gamma_{h}} \\
\gamma=\frac{r(1-R)}{1-r}\end{array}$ & 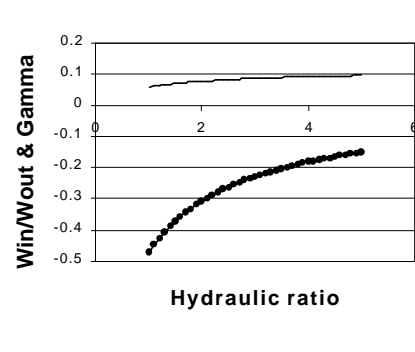 & \begin{tabular}{|l}
$\boldsymbol{-}^{\text {-Gamma }}$ \\
WinWout \\
\end{tabular} \\
\hline 2.4 & $\begin{array}{l}\gamma_{h}=\frac{\sigma_{i n}}{\omega_{o}} \\
\gamma_{c}=\frac{N_{o}}{N_{c}} \\
\gamma_{n}=\frac{N_{2}}{N} \\
N_{1}+N_{1}=N_{c}+N_{2}\end{array}$ & $-\Phi_{i n} \frac{\gamma_{c} \gamma_{n}}{\gamma_{n}}$ & $\begin{array}{l}\gamma_{g}=\frac{N_{R}}{N_{s}} \\
\bar{\varpi}_{s}=\bar{\varpi}_{c} \\
\bar{\varpi}_{a}=\bar{\varpi}_{\text {in }} \\
\bar{\varpi}_{R}=\bar{\varpi}_{\text {out }}\end{array}$ & $\frac{\Phi_{i n}\left(\gamma_{g}+1\right)-\Phi_{c}}{\gamma_{g}+1}$ & $\frac{\gamma_{h}\left(\gamma_{g}+1\right)}{\gamma_{h}\left(\gamma_{g}+1\right)+\gamma_{c} \gamma_{n}}$ & $\begin{array}{c}R=\frac{\gamma_{h}\left(\gamma_{s}+1\right)}{\gamma_{h}\left(\gamma_{g}+1\right)+\gamma_{c} \gamma_{n}} \\
r=\frac{\gamma_{c} \gamma_{n_{2}}}{\gamma_{h}} \\
\gamma=\frac{r(1-R)}{1-r}\end{array}$ & 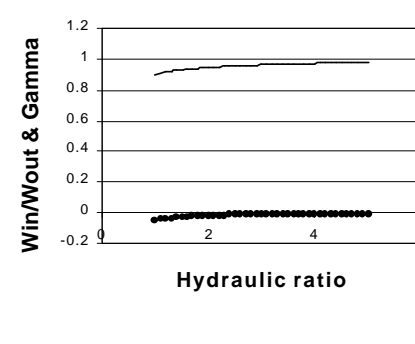 & 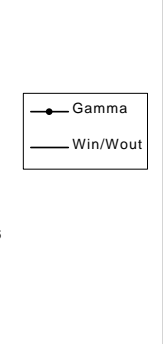 \\
\hline 2.5 & $\begin{array}{l}\gamma_{h}=\frac{\bar{\omega}_{n}}{\omega_{o}} \\
\gamma_{c}=\frac{N_{o}}{N_{c}} \\
\gamma_{n}=\frac{N_{2}}{N} \\
N_{1}+N_{1}=N_{c}+N_{2}\end{array}$ & $-\bar{\sigma}_{i n} \frac{\gamma_{c} \gamma_{n}}{\gamma_{n}}$ & $\begin{array}{l}\gamma_{g}=\frac{N_{R}}{N_{s}} \\
\varpi_{s}=\varpi_{c} \\
\varpi_{a}=\bar{\varpi}_{\text {out }} \\
\bar{\varpi}_{R}=\bar{\varpi}_{\text {in }}\end{array}$ & $\frac{\overline{\boldsymbol{\Phi}}_{c}+\gamma_{g} \overline{\boldsymbol{\varpi}}_{i n}}{\gamma_{g}+1}$ & $\frac{\gamma_{h}\left(\gamma_{g}+1\right)}{\gamma_{h} \gamma_{g}-\gamma_{c} \gamma_{n}}$ & $\begin{array}{c}R=\frac{\gamma_{h}\left(\gamma_{g}+1\right)}{\gamma_{h} \gamma_{g}-\gamma_{c} \gamma_{n}} \\
r=\frac{\gamma_{c} \gamma_{n}}{\gamma_{h}} \\
\gamma=\frac{r(1-R)}{1-r}\end{array}$ & 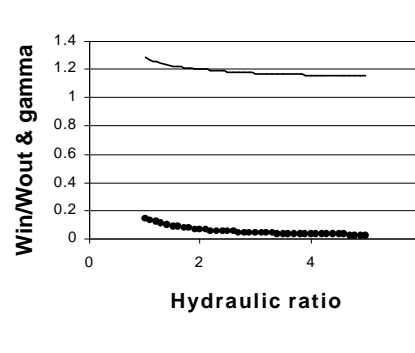 & \begin{tabular}{|l} 
- - Gamma \\
WinNout \\
\end{tabular} \\
\hline 2.6 & $\begin{array}{l}\gamma_{h}=\frac{\bar{\omega}_{n}}{\bar{\omega}_{o}} \\
\gamma_{c}=N_{o} \\
N_{c} \\
\gamma_{n}=\frac{N_{2}}{N} \\
N_{o}+N_{1}=N_{c}+N_{2}\end{array}$ & $-\Phi_{i n} \frac{\gamma_{c} \gamma_{n}}{\gamma_{h}}$ & $\begin{array}{l}\gamma_{g}=\frac{N_{R}}{N_{s}} \\
\varpi_{s}=\varpi_{o u t} \\
\varpi_{a}=\varpi_{c} \\
\varpi_{R}=\bar{\varpi}_{\text {in }}\end{array}$ & $\gamma_{g}\left(\Phi_{c}-\Phi_{i n}\right)+\Phi_{c}$ & $-\frac{\gamma_{h}}{\gamma_{g} \gamma_{h}+\gamma_{c} \gamma_{n}\left(\gamma_{g}+1\right)}$ & $\begin{array}{c}R=-\frac{\gamma_{h}}{\gamma_{s} \gamma_{n}+\gamma_{\gamma_{n}}\left(\gamma_{s}+1\right)} \\
r=\frac{\gamma_{c} \gamma_{n}}{\gamma_{h}} \\
\gamma=\frac{r(1-R)}{1-r}\end{array}$ & 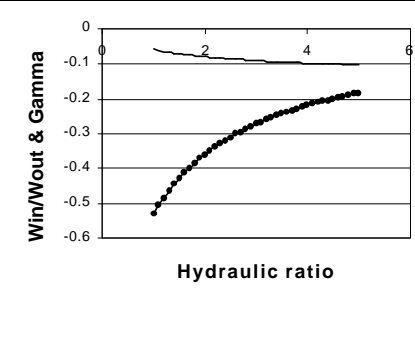 & \begin{tabular}{|l|}
$\boldsymbol{F}^{- \text {Carmma }}$ \\
Winowout \\
\end{tabular} \\
\hline
\end{tabular}




\begin{tabular}{|c|c|c|c|c|c|c|c|c|}
\hline Config. & input variables & $\omega_{c}$ & output variables & $\omega_{\text {out }}$ & $\omega_{n} / \omega_{\text {out }}$ & Power split & Curves & \\
\hline 3.1 & $\begin{array}{l}\gamma_{h}=\frac{\bar{\varpi}_{i n}}{\bar{\varpi}_{o}} \\
\gamma_{c}=\frac{N_{o}}{N_{c}} \\
N_{o}<N_{c}\end{array}$ & $-\varpi_{i n} \frac{\gamma_{c}}{\gamma_{h}}$ & $\begin{array}{l}\gamma_{g}=\frac{N_{R}}{N_{s}} \\
\varpi_{s}=\bar{\varpi}_{\text {in }} \\
\varpi_{a}=\varpi_{\text {out }} \\
\varpi_{R}=\varpi_{c}\end{array}$ & $\frac{\bar{\varpi}_{i n}+\gamma_{g} \bar{\varpi}_{c}}{\gamma_{g}+1}$ & $\frac{\gamma_{h}\left(\gamma_{g}+1\right)}{\gamma_{h}-\gamma_{c} \gamma_{g}}$ & $\begin{array}{c}R=\frac{\gamma_{h}\left(\gamma_{g}+1\right)}{\gamma_{h}-\gamma_{c} \gamma_{g}} \\
r=-\frac{\gamma_{c}}{\gamma_{h}} \\
\gamma=\frac{r(1-R)}{1-r}\end{array}$ & $\sum_{\text {ulic ratio }}$ & 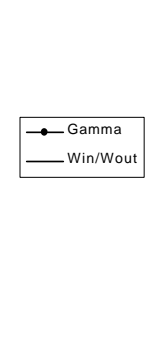 \\
\hline 3.2 & $\begin{array}{l}\gamma_{h}=\frac{\bar{\varpi}_{i n}}{\bar{\varpi}_{o}} \\
\gamma_{c}=\frac{N_{o}}{N_{c}} \\
N_{o}<N_{c}\end{array}$ & $-\bar{\sigma}_{i n} \frac{\gamma_{c}}{\gamma_{h}}$ & $\begin{array}{l}\gamma_{g}=\frac{N_{R}}{N_{s}} \\
\varpi_{s}=\varpi_{\text {in }} \\
\varpi_{a}=\varpi_{c} \\
\varpi_{R}=\varpi_{\text {out }}\end{array}$ & $\frac{\Phi_{c}\left(\gamma_{g}+1\right)-\Phi_{i n}}{\gamma_{g}}$ & $-\frac{\gamma_{g} \gamma_{h}}{\gamma_{h}+\gamma_{c}\left(\gamma_{g}+1\right)}$ & $\begin{array}{c}R=-\frac{\gamma_{\gamma} \gamma_{h}}{\gamma_{h}+\gamma_{c}\left(\gamma_{g}+1\right)} \\
r=-\frac{\gamma_{c}}{\gamma_{h}} \\
\gamma=\frac{r(1-R)}{1-r}\end{array}$ & 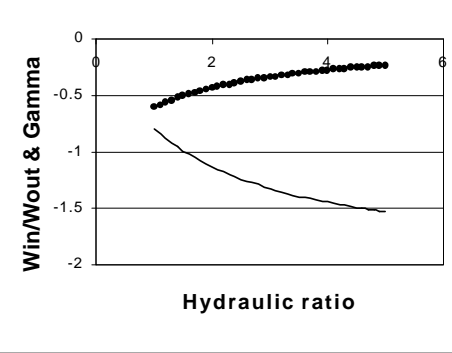 & 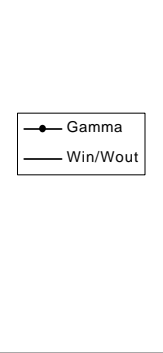 \\
\hline 3.3 & $\begin{array}{l}\gamma_{h}=\frac{\Phi_{i n}}{\Phi_{o}} \\
\gamma_{c}=\frac{N_{o}}{N_{c}} \\
N_{o}<N_{c}\end{array}$ & $-\bar{\sigma}_{i n} \frac{\gamma_{c}}{\gamma_{h}}$ & $\begin{array}{l}\gamma_{g}=\frac{N_{R}}{N_{s}} \\
\varpi_{s}=\varpi_{\text {out }} \\
\varpi_{a}=\varpi_{\text {in }} \\
\varpi_{R}=\bar{\varpi}_{c}\end{array}$ & $\Phi_{i n}\left(\gamma_{g}+1\right)-\gamma_{g} \Phi_{c}$ & $\frac{\gamma_{h}}{\gamma_{h}\left(\gamma_{g}+1\right)+\gamma_{c} \gamma_{g}}$ & $\begin{array}{c}R=\frac{\gamma_{h}}{\gamma_{h}\left(\gamma_{g}+1\right)+\gamma_{c} \gamma_{g}} \\
r=-\frac{\gamma_{c}}{\gamma_{h}} \\
\gamma=\frac{r(1-R)}{1-r}\end{array}$ & 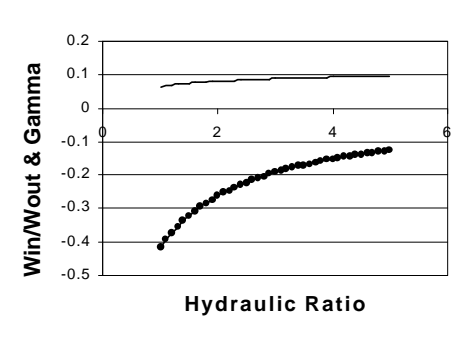 & 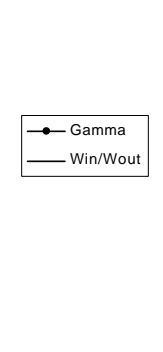 \\
\hline 3.4 & $\begin{array}{l}\gamma_{h}=\frac{\bar{\varpi}_{i n}}{\bar{\varpi}_{o}} \\
\gamma_{c}=\frac{N_{o}}{N_{c}} \\
N_{o}<N_{c}\end{array}$ & $-\boldsymbol{\sigma}_{i n} \frac{\gamma_{c}}{\gamma_{h}}$ & $\begin{array}{l}\gamma_{g}=\frac{N_{R}}{N_{s}} \\
\varpi_{s}=\bar{\varpi}_{c} \\
\varpi_{a}=\varpi_{\text {in }} \\
\varpi_{R}=\varpi_{\text {out }}\end{array}$ & $\frac{\Phi_{i n}\left(\gamma_{g}+1\right)-\Phi_{c}}{\gamma_{g}+1}$ & $\frac{\gamma_{h}\left(\gamma_{s}+1\right)}{\gamma_{h}\left(\gamma_{g}+1\right)+\gamma_{c}}$ & $\begin{array}{c}R=\frac{\gamma_{h}\left(\gamma_{g}+1\right)}{\gamma_{h}\left(\gamma_{g}+1\right)+\gamma_{c}} \\
r=-\frac{\gamma_{c}}{\gamma_{h}} \\
\gamma=\frac{r(1-R)}{1-r}\end{array}$ & 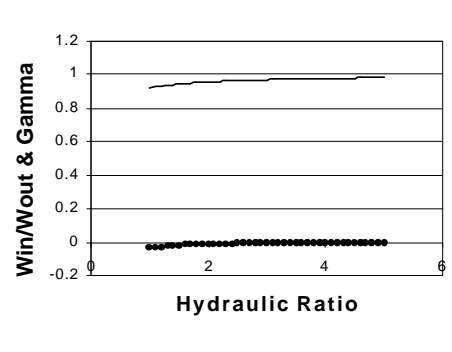 & 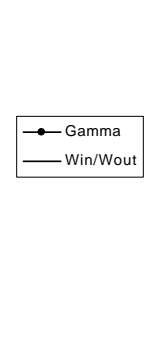 \\
\hline
\end{tabular}




\begin{tabular}{|c|c|c|c|c|c|c|c|c|}
\hline Config. & input variables & $\omega_{\omega_{s}}$ & output variables & $\omega_{\text {out }}$ & $\omega_{n} / \omega_{o u t}$ & Power split & Curves & \\
\hline 3.5 & $\begin{array}{l}\gamma_{h}=\frac{\Phi_{i n}}{\Phi_{o}} \\
\gamma_{c}=\frac{N_{o}}{N_{c}} \\
N_{o}<N_{c}\end{array}$ & $-\bar{\varpi}_{i n} \frac{\gamma_{c}}{\gamma_{h}}$ & $\begin{array}{l}\gamma_{g}=\frac{N_{R}}{N_{s}} \\
\bar{\varpi}_{s}=\bar{\varpi}_{c} \\
\bar{\varpi}_{a}=\bar{\varpi}_{\text {out }} \\
\bar{\varpi}_{R}=\bar{\varpi}_{\text {in }}\end{array}$ & $\frac{\bar{\Phi}_{c}+\gamma_{g} \bar{\Phi}_{i n}}{\gamma_{g}+1}$ & $\frac{\gamma_{h}\left(\gamma_{g}+1\right)}{\gamma_{h} \gamma_{g}-\gamma_{c}}$ & $\begin{aligned} R & =\frac{\gamma_{h}\left(\gamma_{g}+1\right)}{\gamma_{h} \gamma_{g}-\gamma_{c}} \\
r & =-\frac{\gamma_{c}}{\gamma_{h}} \\
\gamma & =\frac{r(1-R)}{1-r}\end{aligned}$ & 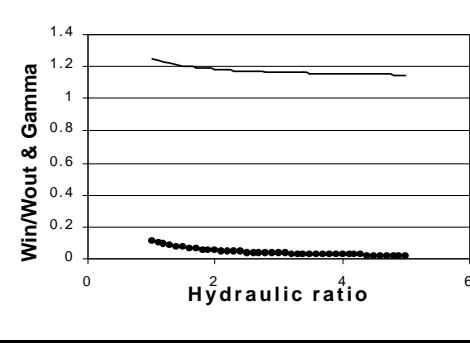 & 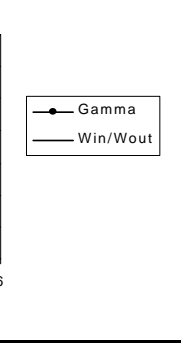 \\
\hline 3.6 & $\begin{array}{l}\gamma_{h}=\frac{\bar{\varpi}_{i n}}{\bar{\varpi}_{o}} \\
\gamma_{c}=\frac{N_{o}}{N_{c}} \\
N_{o}<N_{c}\end{array}$ & $-\bar{\sigma}_{i n} \frac{\gamma_{c}}{\gamma_{h}}$ & $\begin{array}{l}\gamma_{g}=\frac{N_{R}}{N_{s}} \\
\varpi_{s}=\varpi_{\text {out }} \\
\varpi_{a}=\varpi_{c} \\
\varpi_{R}=\varpi_{\text {in }}\end{array}$ & $\gamma_{g}\left(\Phi_{c}-\bar{\Phi}_{i n}\right)+\Phi_{c}$ & $-\frac{\gamma_{h}}{\gamma_{s} \gamma_{h}+\gamma_{c}\left(\gamma_{s}+1\right)}$ & $\begin{array}{c}R=-\frac{\gamma_{h}}{\gamma_{g} \gamma_{h}+\gamma_{c}\left(\gamma_{g}+1\right)} \\
r=-\frac{\gamma_{c}}{\gamma_{h}} \\
\gamma=\frac{r(1-R)}{1-r}\end{array}$ & 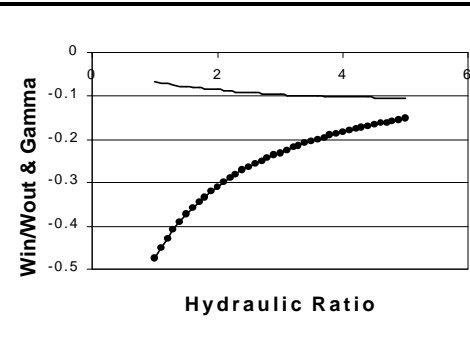 & \begin{tabular}{|l}
$\vec{\nabla}^{\text {Gamman }}$ \\
Winnout
\end{tabular} \\
\hline 4.1 & $\begin{array}{l}\gamma_{h}=\frac{\Phi_{i n}}{\Phi_{o}} \\
\gamma_{c}=\frac{N_{o}}{N_{c}} \\
N_{o}>N_{c}\end{array}$ & $-\bar{\sigma}_{i n} \frac{\gamma_{c}}{\gamma_{h}}$ & $\begin{array}{l}\gamma_{g}=\frac{N_{R}}{N_{s}} \\
\bar{\varpi}_{s}=\bar{\varpi}_{\text {in }} \\
\varpi_{a}=\bar{\varpi}_{\text {out }} \\
\bar{\varpi}_{R}=\bar{\varpi}_{c}\end{array}$ & $\frac{\bar{\varpi}_{i n}+\gamma_{g} \bar{\varpi}_{c}}{\gamma_{g}+1}$ & $\frac{\gamma_{h}\left(\gamma_{g}+1\right)}{\gamma_{h}-\gamma_{c} \gamma_{g}}$ & $\begin{aligned} R & =\frac{\gamma_{h}\left(\gamma_{g}+1\right)}{\gamma_{h}-\gamma_{c} \gamma_{g}} \\
r & =-\frac{\gamma_{c}}{\gamma_{h}} \\
\gamma & =\frac{r(1-R)}{1-r}\end{aligned}$ & 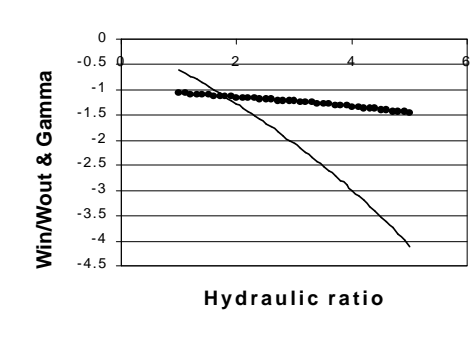 & 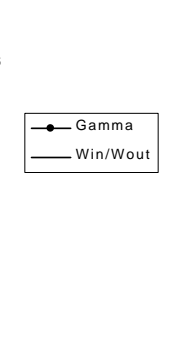 \\
\hline 4.2 & $\begin{array}{l}\gamma_{h}=\frac{\Phi_{i n}}{\Phi_{o}} \\
\gamma_{c}=\frac{N_{o}}{N_{c}} \\
N_{o}>N_{c}\end{array}$ & $-\bar{\omega}_{i n} \frac{\gamma_{c}}{\gamma_{h}}$ & $\begin{array}{l}\gamma_{g}=\frac{N_{R}}{N_{s}} \\
\bar{\varpi}_{s}=\bar{\varpi}_{i n} \\
\bar{\varpi}_{a}=\bar{\varpi}_{c} \\
\bar{\omega}_{R}=\bar{\varpi}_{\text {out }}\end{array}$ & $\frac{\boldsymbol{\Phi}_{c}\left(\gamma_{g}+1\right)-\Phi_{i n}}{\gamma_{g}}$ & $-\frac{\gamma_{g} \gamma_{h}}{\gamma_{h}+\gamma_{c}\left(\gamma_{g}+1\right)}$ & $\begin{array}{c}R=-\frac{\gamma_{g} \gamma_{h}}{\gamma_{h}+\gamma_{c}\left(\gamma_{g}+1\right)} \\
r=-\frac{\gamma_{c}}{\gamma_{h}} \\
\gamma=\frac{r(1-R)}{1-r}\end{array}$ & 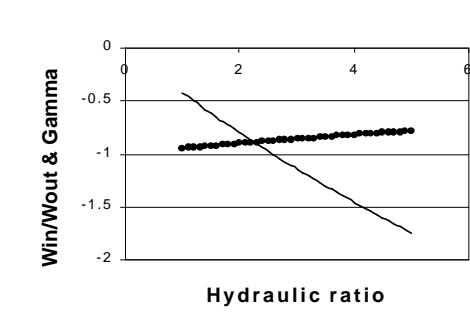 & 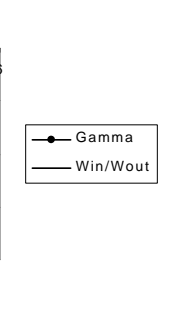 \\
\hline
\end{tabular}




\begin{tabular}{|c|c|c|c|c|c|c|c|c|}
\hline Sonfig & input variables & $\omega_{c}$ & output variables & $\omega_{\text {out }}$ & $\omega_{n}^{\prime} \omega_{\text {out }}$ & Power split & Curves & \\
\hline 4.3 & $\begin{array}{l}\gamma_{h}=\frac{\bar{\varpi}_{i n}}{\bar{\varpi}_{o}} \\
\gamma_{c}=\frac{N_{o}}{N_{c}} \\
N_{o}>N_{c}\end{array}$ & $-\bar{\varpi}_{i n} \frac{\gamma_{c}}{\gamma_{h}}$ & $\begin{array}{l}\gamma_{g}=\frac{N_{R}}{N_{s}} \\
\bar{\varpi}_{s}=\bar{\varpi}_{\text {out }} \\
\bar{\omega}_{a}=\bar{\varpi}_{\text {in }} \\
\bar{\omega}_{R}=\bar{\varpi}_{c}\end{array}$ & $\Phi_{i n}\left(\gamma_{g}+1\right)-\gamma_{g} \Phi_{c}$ & $\frac{\gamma_{h}}{\gamma_{h}\left(\gamma_{g}+1\right)+\gamma_{c} \gamma_{g}}$ & $\begin{array}{c}R=\frac{\gamma_{h}}{\gamma_{h}\left(\gamma_{g}+1\right)+\gamma_{c} \gamma_{g}} \\
r=-\frac{\gamma_{c}}{\gamma_{h}} \\
\gamma=\frac{r(1-R)}{1-r}\end{array}$ & 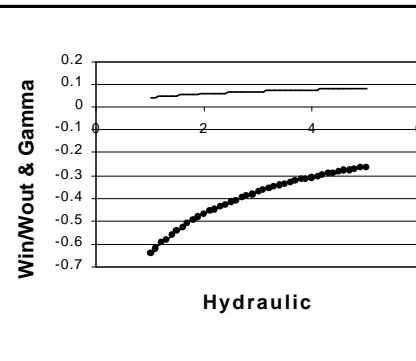 & 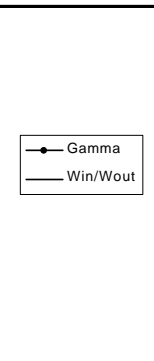 \\
\hline 4.4 & $\begin{array}{l}\gamma_{h}=\frac{\bar{\varpi}_{i n}}{\bar{\varpi}_{o}} \\
\gamma_{c}=\frac{N_{o}}{N_{c}} \\
N_{o}>N_{c}\end{array}$ & $-\bar{\sigma}_{i n} \frac{\gamma_{c}}{\gamma_{h}}$ & $\begin{array}{l}\gamma_{g}=\frac{N_{R}}{N_{s}} \\
\varpi_{s}=\bar{\varpi}_{c} \\
\varpi_{a}=\varpi_{\text {in }} \\
\varpi_{R}=\varpi_{\text {out }}\end{array}$ & $\frac{\Phi_{i n}\left(\gamma_{g}+1\right)-\Phi_{c}}{\gamma_{g}+1}$ & $\frac{\gamma_{h}\left(\gamma_{g}+1\right)}{\gamma_{h}\left(\gamma_{g}+1\right)+\gamma_{c}}$ & $\begin{array}{c}R=\frac{\gamma_{h}\left(\gamma_{g}+1\right)}{\gamma_{h}\left(\gamma_{g}+1\right)+\gamma_{c}} \\
r=-\frac{\gamma_{c}}{\gamma_{h}} \\
\gamma=\frac{r(1-R)}{1-r}\end{array}$ & 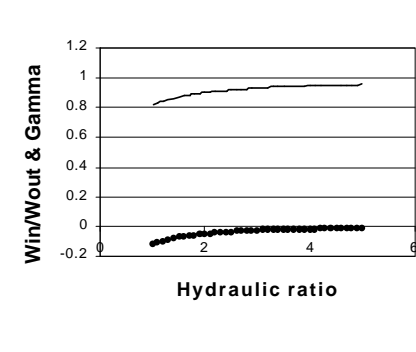 & 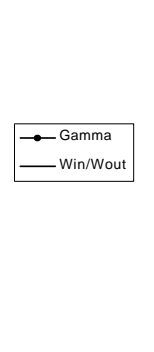 \\
\hline 4.5 & $\begin{array}{l}\gamma_{h}=\frac{\Phi_{i n}}{\Phi_{o}} \\
\gamma_{c}=\frac{N_{o}}{N_{c}} \\
N_{o}>N_{c}\end{array}$ & $-\bar{\omega}_{i n} \frac{\gamma_{c}}{\gamma_{h}}$ & $\begin{array}{l}\gamma_{g}=\frac{N_{R}}{N_{s}} \\
\varpi_{s}=\varpi_{c} \\
\varpi_{a}=\varpi_{\text {out }} \\
\varpi_{R}=\bar{\varpi}_{\text {in }}\end{array}$ & $\frac{\varpi_{c}+\gamma_{g} \bar{\varpi}_{i n}}{\gamma_{g}+1}$ & $\frac{\gamma_{h}\left(\gamma_{g}+1\right)}{\gamma_{h} \gamma_{g}-\gamma_{c}}$ & $\begin{aligned} R & =\frac{\gamma_{h}\left(\gamma_{g}+1\right)}{\gamma_{h} \gamma_{g}-\gamma_{c}} \\
r & =-\frac{\gamma_{c}}{\gamma_{h}} \\
\gamma & =\frac{r(1-R)}{1-r}\end{aligned}$ & 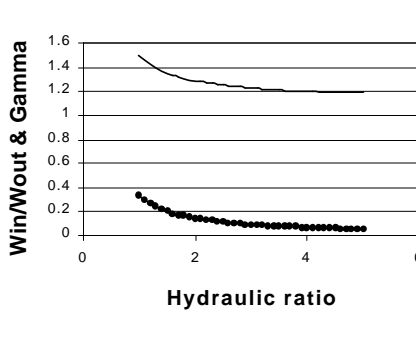 & 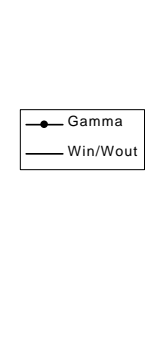 \\
\hline 4.6 & $\begin{array}{l}\gamma_{h}=\frac{\Phi_{i n}}{\bar{\Phi}_{o}} \\
\gamma_{c}=\frac{N_{o}}{N_{c}} \\
N_{o}>N_{c}\end{array}$ & $-\bar{\varpi}_{i n} \frac{\gamma_{c}}{\gamma_{h}}$ & $\begin{array}{l}\gamma_{g}=\frac{N_{R}}{N_{s}} \\
\varpi_{s}=\bar{\varpi}_{\text {out }} \\
\varpi_{a}=\bar{\varpi}_{c} \\
\varpi_{R}=\bar{\varpi}_{\text {in }}\end{array}$ & $\gamma_{g}\left(\Phi_{c}-\Phi_{i n}\right)+\Phi_{c}$ & $-\frac{\gamma_{h}}{\gamma_{g} \gamma_{h}+\gamma_{c}\left(\gamma_{g}+1\right)}$ & $\begin{array}{c}R=-\frac{\gamma_{h}}{\gamma_{g} \gamma_{h}+\gamma_{c}\left(\gamma_{g}+1\right)} \\
r=-\frac{\gamma_{c}}{\gamma_{h}} \\
\gamma=\frac{r(1-R)}{1-r}\end{array}$ & 幽 & 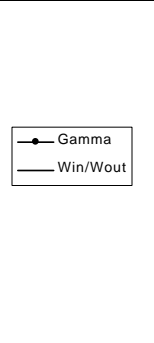 \\
\hline
\end{tabular}




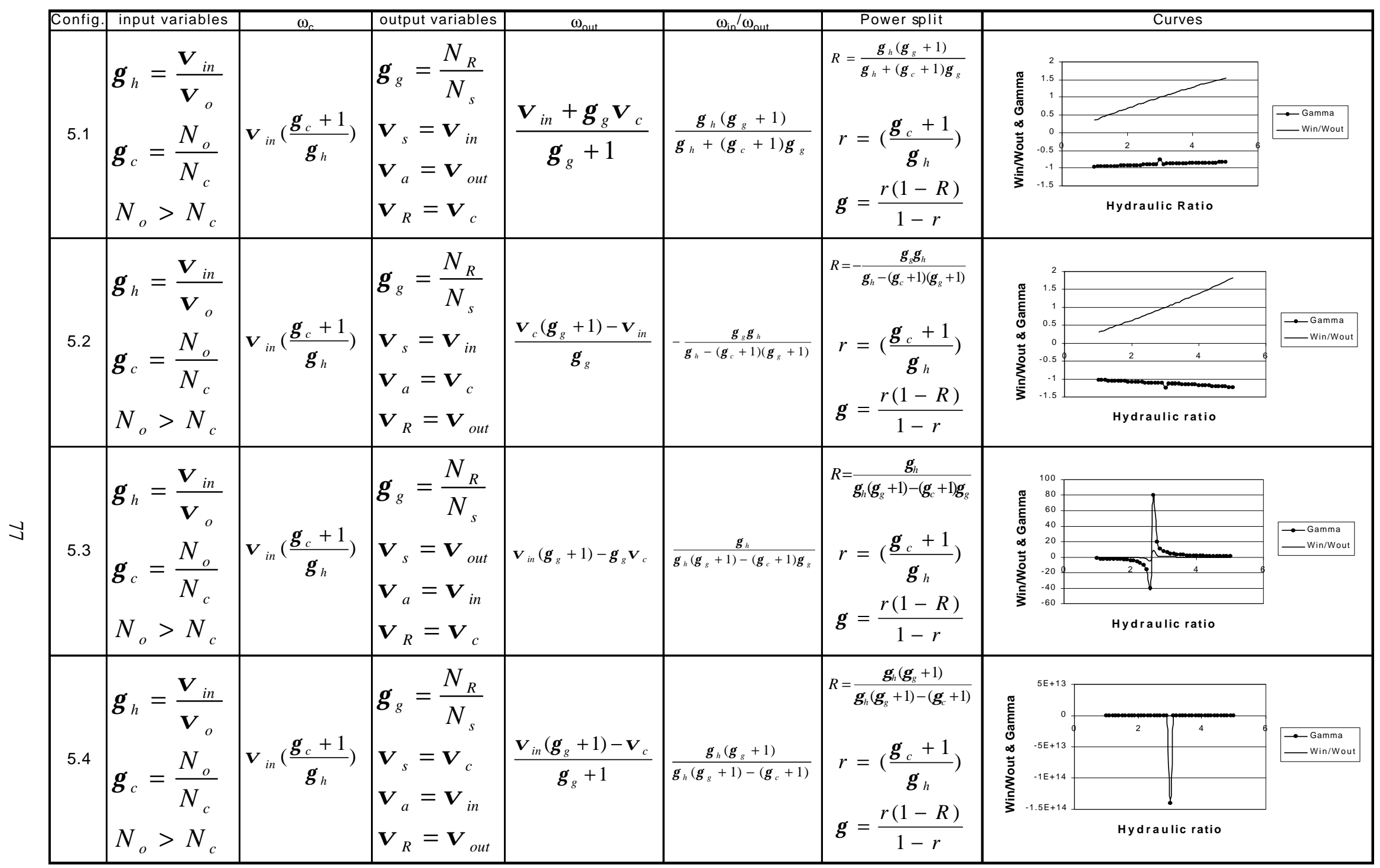




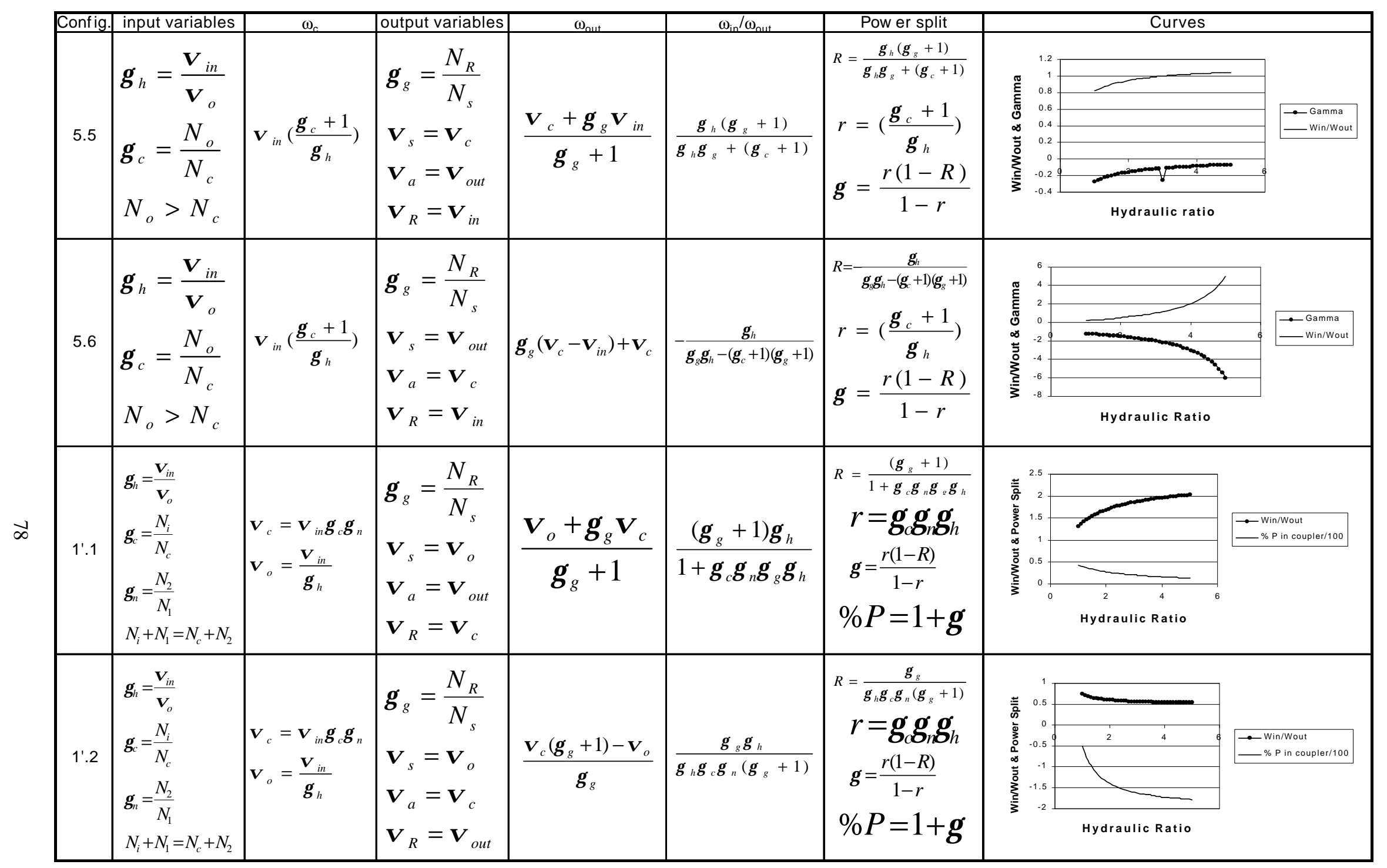




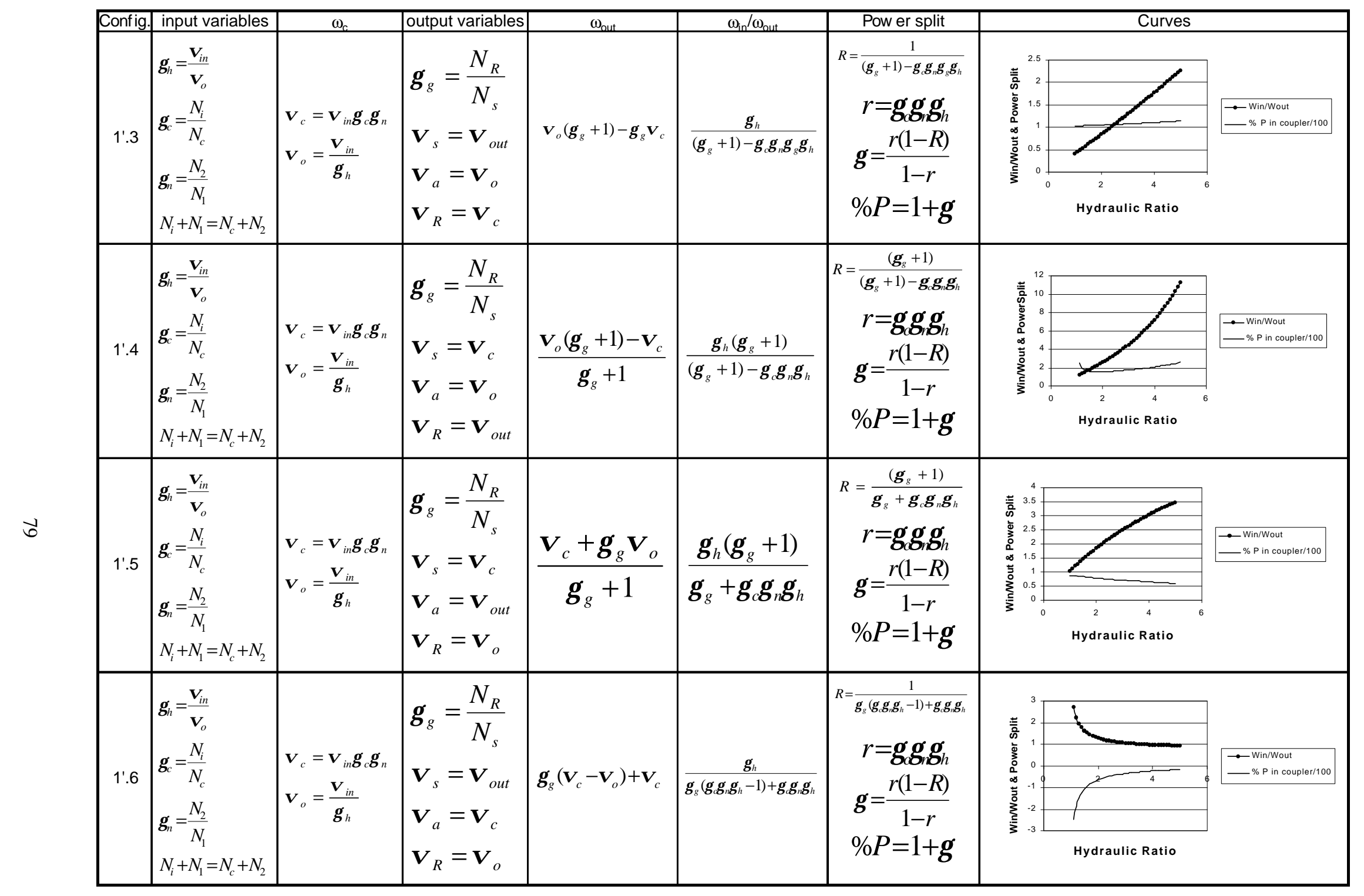




\begin{tabular}{|c|c|c|c|c|c|c|c|c|}
\hline onfig & input variables & $\omega_{s s}$ & output variables & $\omega_{0 \text { out }}$ & $\omega_{0} / \omega_{0 \text { wh }}$ & Pow er split & $\begin{array}{l}\text { Curves } \\
\end{array}$ & \\
\hline $2: 1$ & $\begin{array}{l}\gamma_{h}=\frac{\Phi_{i n}}{\Phi_{o}} \\
\gamma_{c}=\frac{N_{i}}{N_{c}} \\
\gamma_{n}=\frac{N_{2}}{N_{1}} \\
N_{i}+N_{1}=N_{c}+N_{2}\end{array}$ & $\begin{array}{l}\Phi_{c}=-\bar{\Phi}_{i n} \gamma_{c} \gamma_{n} \\
\varpi_{o}=\frac{\Phi_{i n}}{\gamma_{h}}\end{array}$ & $\begin{array}{l}\gamma_{g}=\frac{N_{R}}{N_{s}} \\
\bar{\varpi}_{s}=\bar{\varpi}_{o} \\
\varpi_{a}=\bar{\varpi}_{\text {out }} \\
\varpi_{R}=\varpi_{c}\end{array}$ & $\frac{\bar{\varpi}_{o}+\gamma_{g} \bar{\varpi}_{c}}{\gamma_{g}+1}$ & $\frac{\left(\gamma_{g}+1\right) \gamma_{h}}{1-\gamma_{h} \gamma_{c} \gamma_{n} \gamma_{g}}$ & $\begin{array}{c}R=-\frac{\gamma_{g}}{1+\gamma_{\gamma_{h}} \gamma_{h}\left(\gamma_{s}+1\right)} \\
r=-\gamma_{c} \gamma_{n} \gamma_{h} \\
\gamma=\frac{r(1-R)}{1-r} \\
\% P=1+\gamma\end{array}$ & 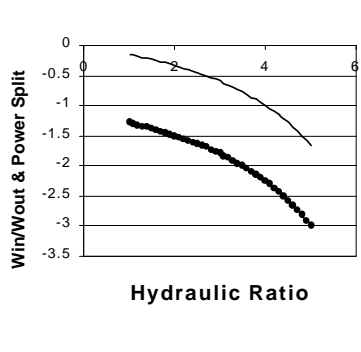 & 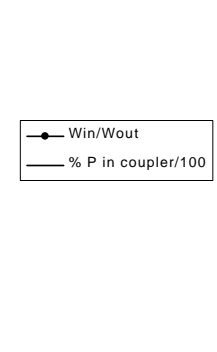 \\
\hline 2'.2 & $\begin{array}{l}\gamma_{h}=\frac{\bar{\omega}_{i n}}{\bar{\sigma}_{o}} \\
\gamma_{c}=\frac{N_{i}}{N_{c}} \\
\gamma_{n}=\frac{N_{2}}{N_{1}} \\
N_{i}+N_{1}=N_{c}+N_{2}\end{array}$ & $\begin{array}{l}\Phi_{c}=-\Phi_{i n} \gamma_{c} \gamma_{n} \\
\varpi_{o}=\frac{\Phi_{i n}}{\gamma_{h}}\end{array}$ & $\begin{array}{l}\gamma_{g}=\frac{N_{R}}{N_{s}} \\
\varpi_{s}=\varpi_{o} \\
\varpi_{a}=\varpi_{c} \\
\varpi_{R}=\varpi_{\text {out }}\end{array}$ & $\frac{\Phi_{c}\left(\gamma_{g}+1\right)-\Phi_{o}}{\gamma_{g}}$ & $-\frac{\gamma_{g} \gamma_{h}}{1+\gamma_{c} \gamma_{n} \gamma_{h}\left(\gamma_{g}+1\right)}$ & $\begin{array}{c}R=-\frac{\gamma_{g}}{1+\gamma_{c} \gamma_{n} \gamma_{h}\left(\gamma_{g}+1\right)} \\
r=-\gamma_{c} \gamma_{n} \gamma_{h} \\
\gamma=\frac{r(1-R)}{1-r} \\
\% P=1+\gamma\end{array}$ & מ & 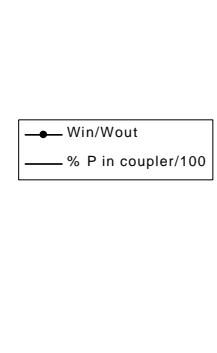 \\
\hline $2: 3$ & $\begin{array}{l}\gamma_{h}=\frac{\sigma_{i n}}{\omega_{o}} \\
\gamma_{c}=\frac{N_{i}}{N_{c}} \\
\gamma_{n}=\frac{N_{2}}{N_{1}} \\
N_{i}+N_{1}=N_{c}+N_{2}\end{array}$ & $\begin{array}{l}\Phi_{c}=-\Phi_{i n} \gamma_{c} \gamma_{n} \\
\varpi_{o}=\frac{\Phi_{i n}}{\gamma_{h}}\end{array}$ & $\begin{array}{l}\gamma_{g}=\frac{N_{R}}{N_{s}} \\
\varpi_{s}=\varpi_{\text {out }} \\
\varpi_{a}=\varpi_{o} \\
\varpi_{R}=\varpi_{c}\end{array}$ & $\Phi_{o}\left(\gamma_{g}+1\right)-\gamma_{g} \Phi$ & $\frac{\gamma_{h}}{\left(\gamma_{g}+1\right)+\gamma_{c} \gamma_{n} \gamma_{g} \gamma_{h}}$ & $\begin{array}{c}R=\frac{1}{\left(\gamma_{s}+1\right)+\gamma_{c} \gamma_{h} \gamma_{g} \gamma_{h}} \\
r=-\gamma_{c} \gamma_{n} \gamma_{h} \\
\gamma=\frac{r(1-R)}{1-r} \\
\% P=1+\gamma\end{array}$ & 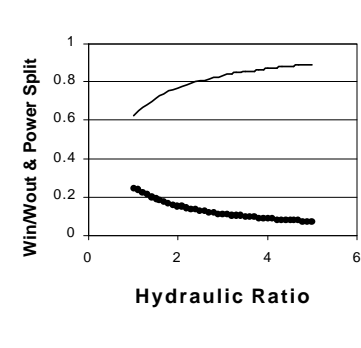 & 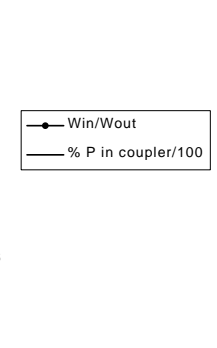 \\
\hline 2'.4 & $\begin{array}{l}\gamma_{h}=\frac{\sigma_{i n}}{\sigma_{o}} \\
\gamma_{c}=\frac{N_{i}}{N_{c}} \\
\gamma_{n}=\frac{N_{2}}{N_{1}} \\
N_{i}+N_{1}=N_{c}+N_{2}\end{array}$ & $\mid \begin{array}{l}\Phi_{c}=-\bar{\varpi}_{i n} \gamma_{c} \gamma_{n} \\
\bar{\varpi}_{o}=\frac{\Phi_{i n}}{\gamma_{h}}\end{array}$ & $\begin{array}{l}\gamma_{g}=\frac{N_{R}}{N_{s}} \\
\varpi_{s}=\varpi_{c} \\
\varpi_{a}=\varpi_{o} \\
\varpi_{R}=\varpi_{\text {out }}\end{array}$ & $\frac{\Phi_{o}\left(\gamma_{g}+1\right)-\Phi_{c}}{\gamma_{g}+1}$ & $\frac{\gamma_{h}\left(\gamma_{g}+1\right)}{\left(\gamma_{g}+1\right)+\gamma_{c} \gamma_{n} \gamma_{h}}$ & $\begin{array}{c}R=\frac{\left(\gamma_{g}+1\right)}{\left(\gamma_{g}+1\right)+\gamma_{\gamma} \gamma_{h} \gamma_{h}} \\
r=-\gamma_{c} \gamma_{n} \gamma_{h} \\
\gamma=\frac{r(1-R)}{1-r} \\
\% P=1+\gamma\end{array}$ & 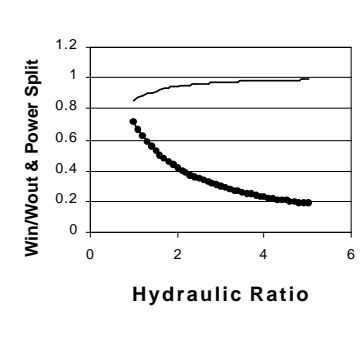 & 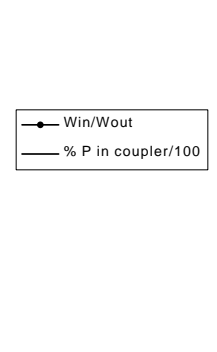 \\
\hline
\end{tabular}




\begin{tabular}{|c|c|c|c|c|c|c|c|c|}
\hline 2.5 & \begin{tabular}{|l} 
input variables \\
$\gamma_{h}=\frac{\varpi_{i n}}{\varpi_{o}}$ \\
$\gamma_{c}=\frac{N_{i}}{N_{c}}$ \\
$\gamma_{n}=\frac{N_{2}}{N_{1}}$ \\
$N_{i}+N_{1}=N_{c}+N_{2}$
\end{tabular} & $\begin{array}{l}\Phi_{c}=-\Phi_{i n} \gamma_{c} \gamma_{n} \\
\varpi_{o}=\frac{\Phi_{i n}}{\gamma_{h}}\end{array}$ & $\begin{array}{l}\text { output variables } \\
\gamma_{g}=\frac{N_{R}}{N_{s}} \\
\varpi_{s}=\bar{\varpi}_{c} \\
\varpi_{a}=\bar{\varpi}_{\text {out }} \\
\varpi_{R}=\bar{\varpi}_{o}\end{array}$ & $\frac{\overline{\boldsymbol{\omega}}_{c}+\gamma_{g} \overline{\boldsymbol{\omega}}_{o}}{\gamma_{g}+1}$ & $\frac{\gamma_{h}\left(\gamma_{g}+1\right)}{\gamma_{g}-\gamma_{c} \gamma_{n} \gamma_{h}}$ & 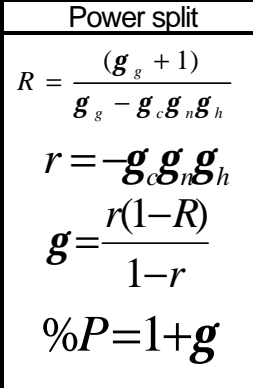 & 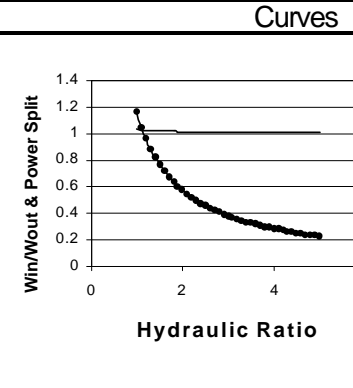 & 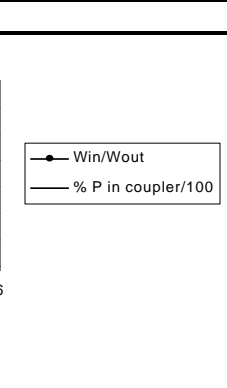 \\
\hline 2.6 & $\begin{array}{l}\gamma_{h}=\frac{\bar{\omega}_{i n}}{\bar{\omega}_{o}} \\
\gamma_{c}=\frac{N_{i}}{N_{c}} \\
\gamma_{n}=\frac{N_{2}}{N_{1}} \\
N_{i}+N_{1}=N_{c}+N_{2}\end{array}$ & $\left\{\begin{array}{l}\Phi_{c}=-\bar{\Phi}_{i n} \gamma_{c} \gamma_{n} \\
\bar{\varpi}_{o}=\frac{\Phi_{i n}}{\gamma_{h}}\end{array}\right.$ & $\begin{array}{l}\gamma_{g}=\frac{N_{R}}{N_{s}} \\
\varpi_{s}=\bar{\varpi}_{\text {out }} \\
\varpi_{a}=\varpi_{c} \\
\varpi_{R}=\varpi_{o}\end{array}$ & $\gamma_{g}\left(\bar{\omega}_{c}-\Phi_{o}\right)+\Phi_{c}$ & $-\frac{\gamma_{h}}{\gamma_{g}\left(\gamma \gamma_{n} \gamma_{h}+1\right)+\gamma_{C} \gamma_{n} \gamma_{h}}$ & $\begin{array}{c}R=\frac{1}{\gamma_{g}\left(\gamma \gamma_{c} \gamma_{h}+1\right)+\gamma_{c} \gamma_{n} \gamma_{n}} \\
r=-\gamma_{c} \gamma_{n} \gamma_{h} \\
\gamma=\frac{r(1-R)}{1-r} \\
\% P=1+\gamma\end{array}$ & 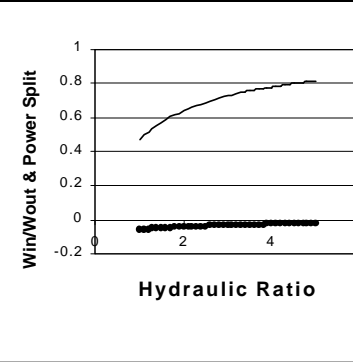 & 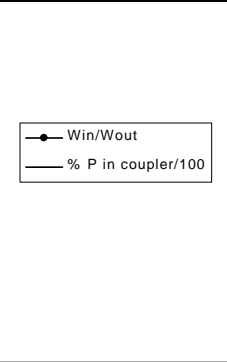 \\
\hline 3:1 & $\begin{array}{l}\gamma_{h}=\frac{\bar{\varpi}_{i n}}{\bar{\varpi}_{o}} \\
\gamma_{c}=\frac{N_{i}}{N_{c}} \\
N_{i}<N_{c}\end{array}$ & $\begin{array}{l}\varpi_{c}=-\varpi_{i n} \gamma_{c} \\
\varpi_{o}=\frac{\varpi_{i n}}{\gamma_{h}}\end{array}$ & $\begin{array}{l}\gamma_{g}=\frac{N_{R}}{N_{s}} \\
\varpi_{s}=\bar{\varpi}_{o} \\
\varpi_{a}=\varpi_{\text {out }} \\
\varpi_{R}=\varpi_{c}\end{array}$ & $\frac{\bar{\varpi}_{o}+\gamma_{g} \bar{\varpi}_{c}}{\gamma_{g}+1}$ & $\frac{\gamma_{h}\left(\gamma_{g}+1\right)}{1-\gamma_{c} \gamma_{g} \gamma_{h}}$ & $\begin{array}{c}R=\frac{\left(\gamma_{g}+1\right)}{1-\gamma_{c} \gamma_{g} \gamma_{h}} \\
r=-\gamma_{c} \gamma_{h} \\
\gamma=\frac{r(1-R)}{1-r} \\
\% P=1+\gamma\end{array}$ & 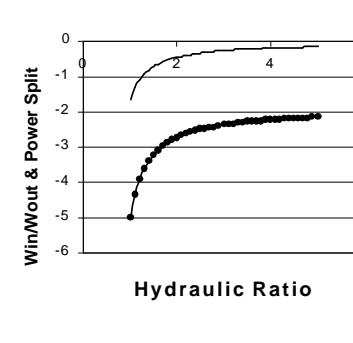 & 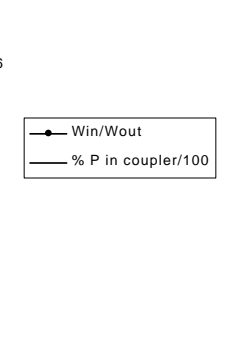 \\
\hline 3'.2 & $\begin{array}{l}\gamma_{h}=\frac{\bar{\varpi}_{i n}}{\Phi_{o}} \\
\gamma_{c}=\frac{N_{i}}{N_{c}} \\
N_{i}<N_{c}\end{array}$ & $\begin{array}{c}\bar{\varpi}_{c}=-\bar{\varpi}_{i n} \gamma_{c} \\
\bar{\varpi}_{o}=\frac{\varpi_{i n}}{\gamma_{h}}\end{array}$ & $\begin{array}{l}\gamma_{g}=\frac{N_{R}}{N_{s}} \\
\varpi_{s}=\varpi_{o} \\
\varpi_{a}=\varpi_{c} \\
\varpi_{R}=\varpi_{\text {out }}\end{array}$ & $\frac{\Phi_{c}\left(\gamma_{g}+1\right)-\bar{\Phi}_{o}}{\gamma_{g}}$ & $-\frac{\gamma_{g} \gamma_{h}}{1+\gamma_{c} \gamma_{h}\left(\gamma_{g}+1\right)}$ & $\begin{array}{c}R=-\frac{\gamma_{s}}{1+\gamma_{c h}\left(\gamma_{s}+1\right)} \\
r=-\gamma_{c} \gamma_{h} \\
\gamma=\frac{r(1-R)}{1-r} \\
\% P=1+\gamma\end{array}$ & 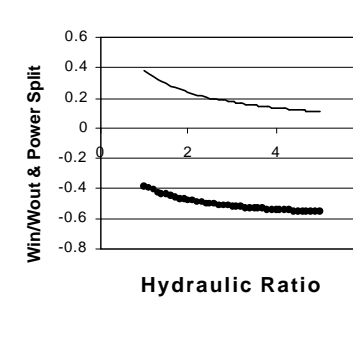 & 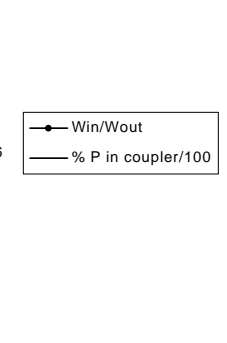 \\
\hline
\end{tabular}




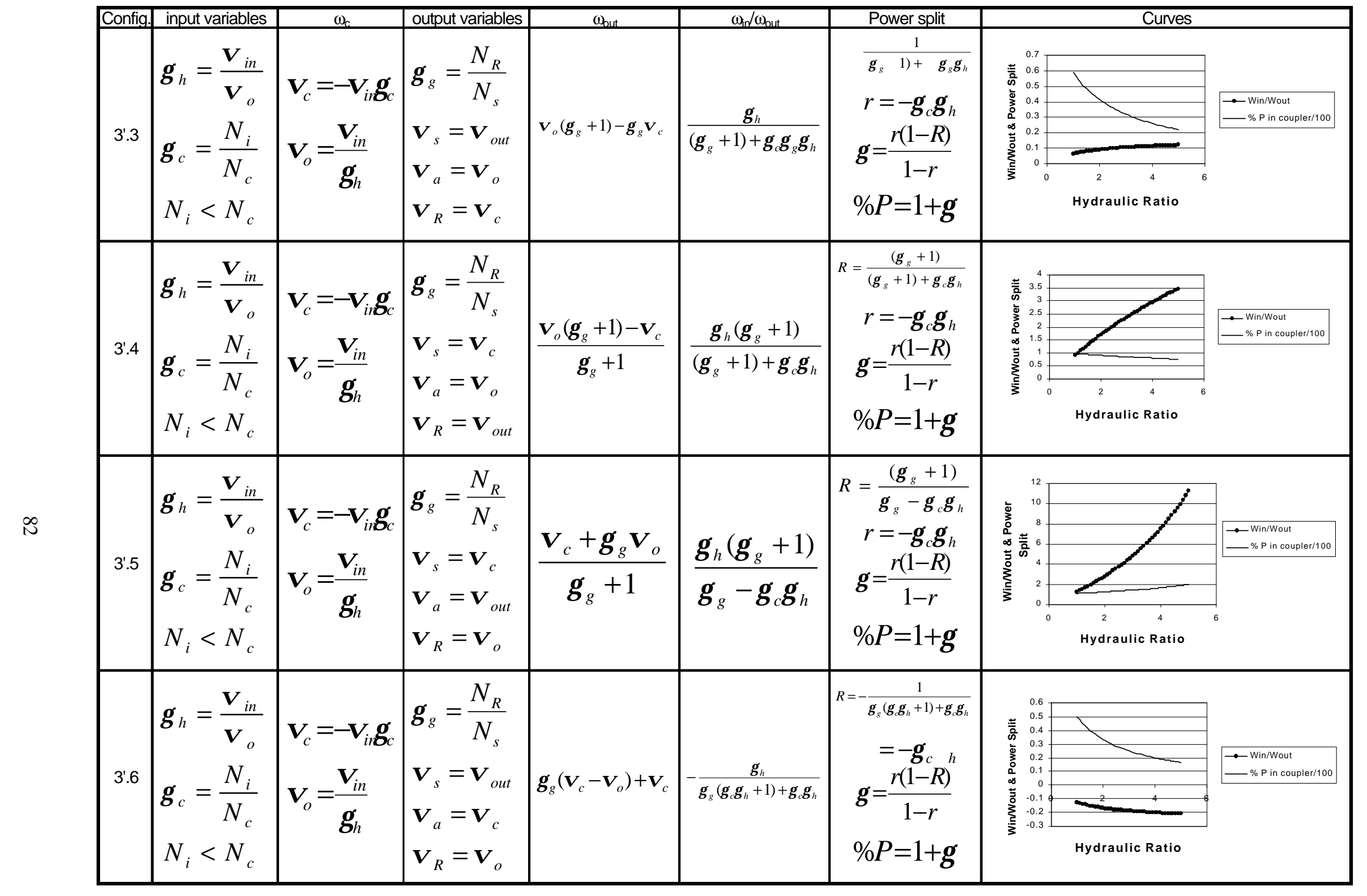




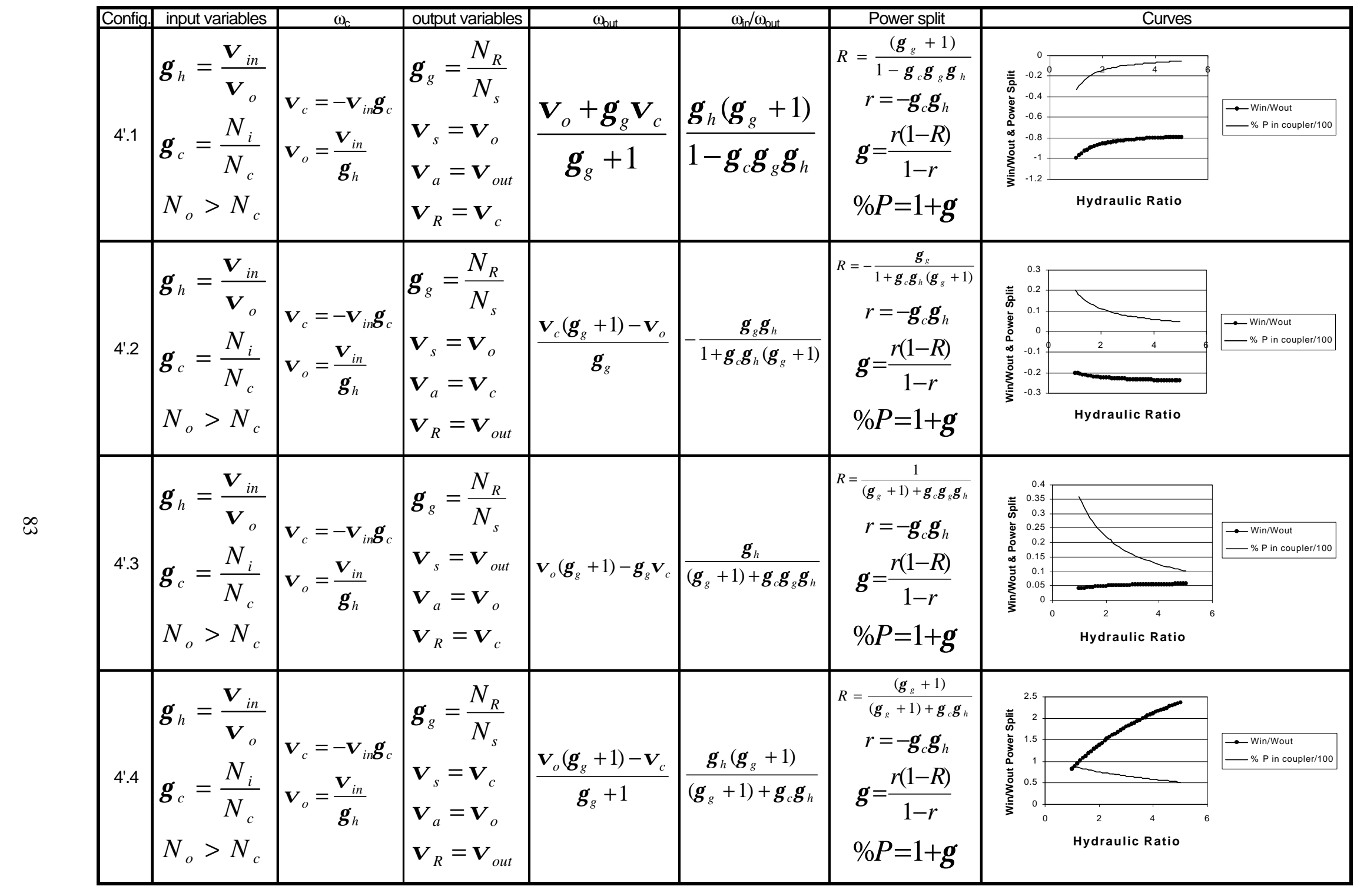




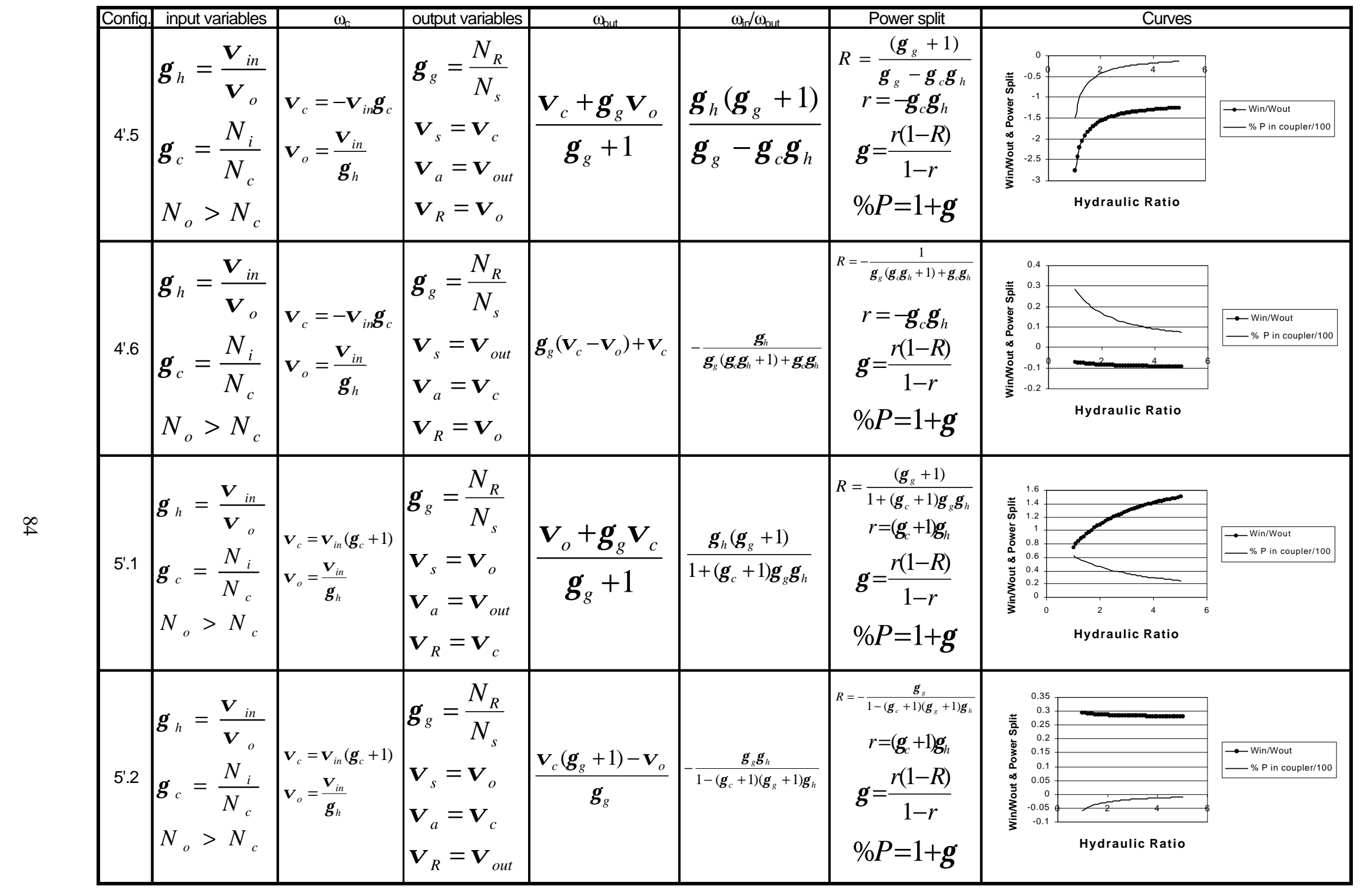




\begin{tabular}{|c|c|c|c|c|c|c|c|c|}
\hline$\overline{\text { Config }}$ & \begin{tabular}{|l|l|} 
input variables \\
\end{tabular} & $\omega_{c}$ & \begin{tabular}{|l} 
output variables \\
\end{tabular} & $\omega_{\text {out }}$ & $\omega_{r} / \omega_{\text {wit }}$ & Power split & Curves & \\
\hline $5^{5} .3$ & $\begin{array}{l}\gamma_{h}=\frac{\overline{\boldsymbol{\sigma}}_{i n}}{\boldsymbol{\Phi}_{o}} \\
\gamma_{c}=\frac{N_{i}}{N_{c}} \\
N_{o}>N_{c}\end{array}$ & $\begin{array}{l}\Phi_{c}=\bar{\Phi}_{i n}\left(\gamma_{c}+1\right) \\
\varpi_{o}=\frac{\Phi_{i n}}{\gamma_{h}}\end{array}$ & $\begin{array}{l}\gamma_{g}=\frac{N_{R}}{N_{s}} \\
\varpi_{s}=\varpi_{o u t} \\
\varpi_{a}=\varpi_{o} \\
\varpi_{R}=\varpi_{c}\end{array}$ & $\Phi_{o}\left(\gamma_{g}+1\right)-\gamma_{g} \Phi_{c}$ & $\frac{\gamma_{h}}{\left(\gamma_{g}+1\right)-\left(\gamma_{c}+1\right) \gamma_{g} \gamma_{h}}$ & $\begin{array}{c}R=\frac{1}{\left(\gamma_{s}+1\right)-\left(\gamma_{c}+1\right) \gamma_{s} \gamma_{n}} \\
r=\left(\gamma_{c}+1\right) \gamma_{h} \\
\gamma=\frac{r(1-R)}{1-r} \\
\% P=1+\gamma\end{array}$ & 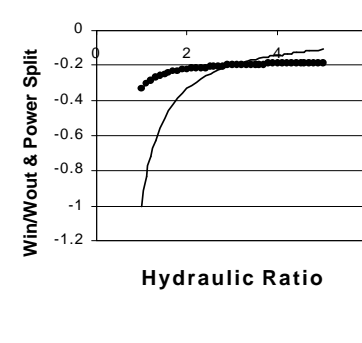 & 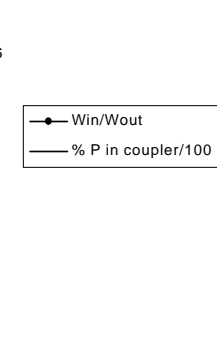 \\
\hline 5.4 & $\begin{array}{l}\gamma_{h}=\frac{\bar{\Phi}_{i n}}{\bar{\Phi}_{o}} \\
\gamma_{c}=\frac{N_{i}}{N_{c}} \\
N_{o}>N_{c}\end{array}$ & $\begin{array}{l}\Phi_{c}=\Phi_{i n}\left(\gamma_{c}+1\right) \\
\varpi_{o}=\frac{\Phi_{i n}}{\gamma_{h}}\end{array}$ & $\begin{array}{l}\gamma_{g}=\frac{N_{R}}{N_{s}} \\
\varpi_{s}=\bar{\varpi}_{c} \\
\varpi_{a}=\varpi_{o} \\
\varpi_{R}=\varpi_{\text {out }}\end{array}$ & $\frac{\Phi_{o}\left(\gamma_{g}+1\right)-\Phi_{c}}{\gamma_{g}+1}$ & $\frac{\gamma_{h}\left(\gamma_{s}+1\right)}{\left(\gamma_{B}+1\right)-\left(\gamma_{c}+1\right) \gamma_{h}}$ & $\begin{array}{c}R=\frac{\left(\gamma_{g}+1\right)}{\left(\gamma_{g}+1\right)-\left(\gamma_{c}+1\right) \gamma_{h}} \\
r=\left(\gamma_{c}+1\right) \gamma_{h} \\
\gamma=\frac{r(1-R)}{1-r} \\
\% P=1+\gamma\end{array}$ & 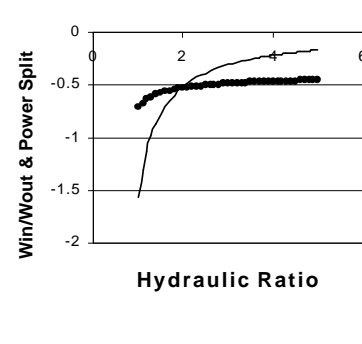 & $\begin{array}{l}- \text { Win'Wout } \\
-\% \text { in coupler/ } 100\end{array}$ \\
\hline 5.5 & $\begin{array}{l}\gamma_{h}=\frac{\bar{\Phi}_{i n}}{\Phi_{o}} \\
\gamma_{c}=\frac{N_{i}}{N_{c}} \\
N_{o}>N_{c}\end{array}$ & $\begin{array}{l}\Phi_{c}=\Phi_{i n}\left(\gamma_{c}+1\right) \\
\Phi_{o}=\frac{\Phi_{i n}}{\gamma_{h}}\end{array}$ & $\begin{array}{l}\gamma_{g}=\frac{N_{R}}{N_{s}} \\
\varpi_{s}=\varpi_{c} \\
\varpi_{a}=\varpi_{\text {out }} \\
\varpi_{R}=\varpi_{o}\end{array}$ & $\frac{\bar{\varpi}_{c}+\gamma_{g} \bar{\varpi}_{o}}{\gamma_{g}+1}$ & $\frac{\gamma_{h}\left(\gamma_{g}+1\right)}{\gamma_{g}+\left(\gamma_{c}+1\right) \gamma_{h}}$ & $\begin{array}{c}R=\frac{\left(\gamma_{g}+1\right)}{\gamma_{g}+\left(\gamma_{c}+1\right) \gamma_{h}} \\
r=\left(\gamma_{c}+1\right) \gamma_{h} \\
\gamma=\frac{r(1-R)}{1-r} \\
\% P=1+\gamma\end{array}$ & $\begin{array}{c}2 \\
\text { Hydraulic Ratio }\end{array}$ & 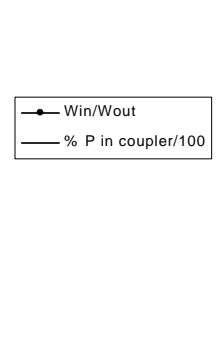 \\
\hline $5^{\prime} \cdot 6$ & $\begin{array}{l}\gamma_{h}=\frac{\Phi_{i n}}{\Phi_{o}} \\
\gamma_{c}=\frac{N_{i}}{N_{c}} \\
N_{o}>N_{c}\end{array}$ & $\begin{array}{l}\Phi_{c}=\Phi_{i n}\left(\gamma_{c}+1\right) \\
\Phi_{o}=\frac{\Phi_{\text {in }}}{\gamma_{h}}\end{array}$ & $\begin{array}{l}\gamma_{g}=\frac{N_{R}}{N_{s}} \\
\varpi_{s}=\varpi_{\text {out }} \\
\varpi_{a}=\bar{\varpi}_{c} \\
\varpi_{R}=\varpi_{o}\end{array}$ & $\gamma_{g}\left(\Phi_{c}-\Phi_{o}\right)+\Phi_{c}$ & $\frac{\gamma_{n}}{\left.\gamma_{r}\left(\gamma_{c}+1\right) \gamma_{n}-1\right)+\left(\gamma_{c}+1 \gamma_{k}\right.}$ & $\begin{array}{c}R=\frac{1}{\gamma_{s}\left(\theta_{c}+1 \gamma_{h}-1\right)+\left(_{c}+1\right) \gamma_{h}} \\
r=\left(\gamma_{c}+1\right) \gamma_{h} \\
\gamma=\frac{r(1-R)}{1-r} \\
\% P=1+\gamma\end{array}$ & 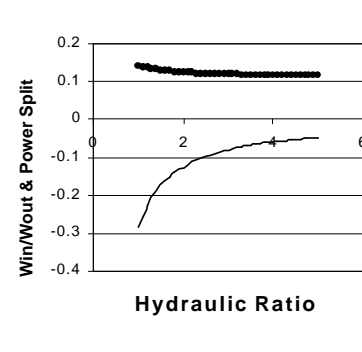 & 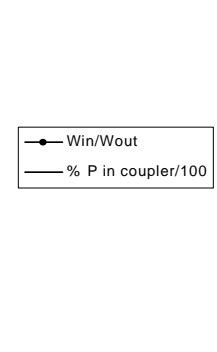 \\
\hline
\end{tabular}




\section{Appendix B: Gear Sizes of Example Hydrodynamic Coupling Conceptual Design}




\begin{tabular}{|c|c|c|c|c|c|c|c|}
\hline \multicolumn{8}{|l|}{425 HP Application with Voith SVN 313 coupling } \\
\hline \multicolumn{8}{|c|}{ Face Width for Planetary and Control Gears using AGMA Bending Strength Formula } \\
\hline Gear & & $\mathrm{N}_{\mathrm{P}}$ & $\mathrm{N}_{\mathrm{S}}$ & $\mathrm{N}_{\mathrm{R}}$ & $\mathrm{N}_{\mathrm{o}}$ & $\mathrm{N}_{\mathrm{c}}$ & $\mathrm{N}_{\text {ldler }}$ \\
\hline Dynamic factor & $K_{v}=\left(A /\left(A+V^{1 / 2}\right)^{B}\right.$ & 1.1207658 & 1.120766 & & 1.092633 & & \\
\hline AGMA transmission accuracy-level number & $Q_{v}$ & 11 & 11 & & 11 & & \\
\hline & $A=50+56(1-B)$ & 92 & 92 & & 92 & & \\
\hline & $B=\left(12-Q_{v}\right)^{2 / 3} / 4$ & 0.25 & 0.25 & & 0.25 & & \\
\hline Pitch line velocity (ft/min) & $\mathrm{V}=\pi^{*} \mathrm{n}_{\mathrm{p}}{ }^{*} \mathrm{~d} / 12$ & 2826 & 2826 & & 1530.75 & & \\
\hline Speed of pinion (rev/min) & $\mathrm{n}_{\mathrm{p}}$ & 7200 & 1800 & & 1800 & & \\
\hline Pitch diameter of pinion (in) & $\mathrm{d}=\mathrm{N} / \mathrm{P}_{\mathrm{d}}$ & 1.5 & 6 & 9 & 3.25 & 10.5 & 3.625 \\
\hline Number of teeth & $\mathrm{N}$ & 12 & 48 & 72 & 26 & 84 & 29 \\
\hline Diametral pitch (teeth per inch) & $P_{d}$ & 8 & 8 & 8 & 8 & 8 & 8 \\
\hline Transmitted pinion torque (in-lb) & $\bar{T}$ & & 8221 & & 6659 & & \\
\hline Transmitted tangential load (lb) & $W_{t}=2^{*} T / d$ & 913.44444 & 913.4444 & & 1365.949 & & \\
\hline Overload factor & $\mathrm{K}_{\mathrm{o}}$ & 1 & & & 1 & & \\
\hline Size factor & $\mathrm{K}_{\mathrm{s}}$ & 1 & & & 1 & & \\
\hline Load distribution factor & $\mathrm{K}_{\mathrm{m}}$ & 1.3 & & & 1.375 & & \\
\hline Rim thickness factor & $\mathrm{K}_{\mathrm{B}}$ & 1 & & & 1 & & \\
\hline Geometry factor for bending strength & $\mathrm{J}$ & 0.32 & & & 0.35 & & \\
\hline AGMA bending strength $\left(\mathrm{Ib} / \mathrm{in}^{2}\right)$ & $\mathrm{s}_{\mathrm{at}}$ & 75000 & & & 75000 & & \\
\hline Stress cycle factor for bending strength & $Y_{N}$ & 0.95 & & & 1 & & \\
\hline Temperature factor & $\mathrm{K}_{\mathrm{T}}$ & 1 & & & 1 & & \\
\hline Reliability factor & $\mathrm{K}_{\mathrm{R}}$ & 1 & & & 1 & & \\
\hline Safety factor for bending strength & $\mathrm{S}_{\mathrm{F}}$ & 1 & & & 1 & & \\
\hline Allowable bending stress number (lb/in $)$ & $\mathrm{S}_{\mathrm{t}}=\mathrm{S}_{\mathrm{at}}{ }^{*} \mathrm{Y}_{\mathrm{N}} /\left(\mathrm{S}_{\mathrm{F}}{ }^{*} \mathrm{~K}_{\mathrm{T}}{ }^{*} \mathrm{~K}_{\mathrm{R}}\right)$ & 71250 & & & 75000 & & \\
\hline Face width (in) & $\mathrm{F}$ & 0.466977 & 0.466977 & 0.466977 & 0.62542 & 0.62542 & 0.62542 \\
\hline
\end{tabular}


425 HP Application with Voith SVN 313 coupling

Face Width for Reduction Gears using AGMA Bending Strength Fundamental Formula Gear

Dynamic factor

AGMA transmission accuracy-level number

\begin{tabular}{|c|c|c|c|c|c|c|c|}
\hline AGMA transmission accuracy-level number & $Q_{v}$ & 11 & 11 & 11 & & & \\
\hline & $A=50+56(1-B)$ & 92 & 92 & 92 & & & \\
\hline & $B=\left(12-Q_{v}\right)^{2 / 3} / 4$ & 0.25 & 0.25 & 0.25 & & & \\
\hline Pitch line velocity (ft/min) & $V=\pi^{*} n_{p}^{*} d / 12$ & 1425.56 & 1853.228 & 4132.449 & & & \\
\hline Speed of pinion (rev/min) & $\mathrm{n}_{\mathrm{p}}$ & 1362 & 1362 & 2078 & & & \\
\hline Pitch diameter of pinion (in) & $d=N / P_{d}$ & 4 & 5.2 & 7.6 & 10.2 & 9 & 6.6 \\
\hline Number of teeth & $\mathrm{N}$ & 20 & 26 & 38 & 51 & 45 & 33 \\
\hline Diametral pitch (teeth per inch) & $P_{d}$ & 5 & 5 & 5 & 5 & 5 & 5 \\
\hline Transmitted pinion torque (in-lb) & $T$ & 13326 & 13326 & 8730 & & & \\
\hline Transmitted tangential load (lb) & $W_{t}=2^{*} T / d$ & 6663 & 5125.385 & 2297.368 & & & \\
\hline Overload factor & $\mathrm{K}_{\mathrm{o}}$ & 11 & 1 & 1 & & & \\
\hline Size factor & $\mathrm{K}_{\mathrm{s}}$ & 1 & 1 & 1 & & & \\
\hline Load distribution factor & $\mathrm{K}_{\mathrm{m}}$ & 1.3 & 1.3 & 1.3 & & & \\
\hline Rim thickness factor & $\mathrm{K}_{\mathrm{B}}$ & 1 & 1 & 1 & & & \\
\hline Geometry factor for bending strength & $\mathrm{J}$ & 0.36 & 0.38 & 0.36 & & & \\
\hline AGMA bending strength $\left(\mathrm{lb} / \mathrm{in}^{2}\right)$ & $\mathrm{S}_{\mathrm{at}}$ & 75000 & 75000 & 75000 & & & \\
\hline Stress cycle factor for bending strength & $\mathrm{Y}_{\mathrm{N}}$ & 1 & 1 & 1 & & & \\
\hline Temperature factor & $\mathrm{K}_{\mathrm{T}}$ & 1 & 1 & 1 & & & \\
\hline Reliability factor & $\overline{K_{R}}$ & 1 & 1 & 1 & & & \\
\hline Safety factor for bending strength & $\mathrm{S}_{\mathrm{F}}$ & 1 & 1 & 1 & & & \\
\hline Allowable bending stress number $\left(\mathrm{lb} / \mathrm{in}^{2}\right)$ & $\mathrm{S}_{\mathrm{t}}=\mathrm{S}_{\mathrm{at}}{ }^{*} \mathrm{Y}_{\mathrm{N}} /\left(\mathrm{S}_{\mathrm{F}}{ }^{*} \mathrm{~K}_{\mathrm{T}}{ }^{*} \mathrm{~K}_{\mathrm{R}}\right)$ & 75000 & 75000 & 75000 & & & \\
\hline Face width (in) & $\mathrm{F}$ & 1.748054 & 1.286681 & 0.631411 & 1.748054 & 1.286681 & 0.631411 \\
\hline
\end{tabular}




\section{Appendix C: Gear Sizes of Example Hydrodynamic Hydrostatic Drive Conceptual Design}




\begin{tabular}{|c|c|c|c|c|c|c|c|}
\hline \multicolumn{8}{|c|}{425 HP Application with Hydrostatic Drive } \\
\hline \multicolumn{8}{|c|}{ Face Width for Planetary and Control Gears using AGMA Bending Strength Formula } \\
\hline Gear & & $\mathrm{N}_{\mathrm{P}}$ & $\mathrm{N}_{S}$ & $\mathrm{~N}_{\mathrm{R}}$ & $\mathrm{N}_{\mathrm{o}}$ & $\mathrm{N}_{\mathrm{c}}$ & $\mathrm{N}_{\text {ldler }}$ \\
\hline Dynamic factor & $K_{v}=\left(A /\left(A+V^{1 / 2}\right)^{B}\right.$ & 1.1067582 & 1.106758 & & 1.089421 & & \\
\hline AGMA transmission accuracy-level number & $Q_{v}$ & 11 & 11 & & 11 & & \\
\hline & $A=50+56(1-B)$ & 92 & 92 & & 92 & & \\
\hline & $\mathrm{B}=\left(12-\mathrm{Q}_{\mathrm{V}}\right)^{2 / 3} / 4$ & 0.25 & 0.25 & & 0.25 & & \\
\hline Pitch line velocity (ft/min) & $\mathrm{V}=\pi^{*} \mathrm{n}_{\mathrm{p}}{ }^{*} \mathrm{~d} / 12$ & 2119.5 & 2119.5 & & 1413 & & \\
\hline Speed of pinion (rev/min) & $\mathrm{n}_{\mathrm{p}}$ & 3600 & 1800 & & 1800 & & \\
\hline Pitch diameter of pinion (in) & $\mathrm{d}=\mathrm{N} / \mathrm{P}_{\mathrm{d}}$ & 2.25 & 4.5 & 9 & 3 & 11 & 4 \\
\hline Number of teeth & $\mathrm{N}$ & 18 & 36 & 72 & 24 & 88 & 32 \\
\hline Diametral pitch (teeth per inch) & $P_{d}$ & 8 & 8 & 8 & 8 & 8 & 8 \\
\hline Transmitted pinion torque (in-lb) & $\mathrm{T}$ & & 8221 & & 6659 & & \\
\hline Transmitted tangential load (lb) & $W_{t}=2^{*} T / d$ & 1217.9259 & 1217.926 & & 1479.778 & & \\
\hline Overload factor & $\mathrm{K}_{\mathrm{o}}$ & 1 & & & 1 & & \\
\hline Size factor & $\mathrm{K}_{\mathrm{s}}$ & 1 & & & 1 & & \\
\hline Load distribution factor & $\mathrm{K}_{\mathrm{m}}$ & 1.3 & & & 1.375 & & \\
\hline Rim thickness factor & $\mathrm{K}_{\mathrm{B}}$ & 1 & & & 1 & & \\
\hline Geometry factor for bending strength & $\mathrm{J}$ & 0.32 & & & 0.35 & & \\
\hline AGMA bending strength $\left(\mathrm{lb} / \mathrm{in}^{2}\right)$ & $\mathrm{s}_{\mathrm{at}}$ & 75000 & & & 75000 & & \\
\hline Stress cycle factor for bending strength & $Y_{N}$ & 0.95 & & & 1 & & \\
\hline Temperature factor & $\mathrm{K}_{\mathrm{T}}$ & 1 & & & 1 & & \\
\hline Reliability factor & $\mathrm{K}_{\mathrm{R}}$ & 1 & & & 1 & & \\
\hline Safety factor for bending strength & $\mathrm{S}_{\mathrm{F}}$ & 1 & & & 1 & & \\
\hline Allowable bending stress number $\left(\mathrm{lb} / \mathrm{in}^{2}\right)$ & $\mathrm{S}_{\mathrm{t}}=\mathrm{S}_{\mathrm{at}}{ }^{*} \mathrm{Y}_{\mathrm{N}} /\left(\mathrm{S}_{\mathrm{F}}{ }^{*} \mathrm{~K}_{\mathrm{T}}{ }^{*} \mathrm{~K}_{\mathrm{R}}\right)$ & 71250 & & & 75000 & & \\
\hline Face width (in) & $\mathrm{F}$ & 0.6148541 & 0.614854 & 0.614854 & 0.675547 & 0.675547 & 0.675547 \\
\hline
\end{tabular}




\begin{tabular}{|c|c|c|c|c|c|c|c|}
\hline \multicolumn{8}{|l|}{425 HP Application with Hydrostatic Drive } \\
\hline \multicolumn{8}{|c|}{ Face Width for Reduction Gears using AGMA Bending Strength Fundamental Formula } \\
\hline Gear & & \begin{tabular}{l|l}
$N_{1}$ \\
\end{tabular} & $\mathrm{~N}_{3}$ & $\mathrm{~N}_{6}$ & $\mathrm{~N}_{2}$ & $\mathrm{~N}_{4}$ & $\mathrm{~N}_{5}$ \\
\hline Dynamic factor & $K_{v}=\left(A /\left(A+V^{1 / 2}\right)^{B}\right.$ & 1.09726 & 1.107167 & 1.116744 & & & \\
\hline AGMA transmission accuracy-level number & $Q_{v}$ & 11 & 11 & 11 & & & \\
\hline & $A=50+56(1-B)$ & 92 & 92 & 92 & & & \\
\hline & $\mathrm{B}=\left(12-\mathrm{Q}_{\mathrm{v}}\right)^{2 / 3} / 4$ & 0.25 & 0.25 & 0.25 & & & \\
\hline Pitch line velocity (ft/min) & $\mathrm{V}=\pi^{\star} \mathrm{n}_{\mathrm{p}}{ }^{*} \mathrm{~d} / 12$ & 1710.672 & 2138.34 & 2609.968 & & & \\
\hline Speed of pinion (rev/min) & $\mathrm{n}_{\mathrm{p}}$ & 1362 & 1362 & 2078 & & & \\
\hline Pitch diameter of pinion (in) & $d=N / P_{d}$ & 4.8 & 6 & 4.8 & 7.2 & 6 & 7.2 \\
\hline Number of teeth & $\mathrm{N}$ & 24 & 30 & 24 & 36 & 30 & 36 \\
\hline Diametral pitch (teeth per inch) & $\overline{P_{d}}$ & 5 & 5 & 5 & 5 & 5 & 5 \\
\hline Transmitted pinion torque (in-lb) & $T$ & 13326 & 13326 & 8730 & & & \\
\hline Transmitted tangential load (lb) & $W_{t}=2^{*} T / d$ & 5552.5 & 4442 & 3637.5 & & & \\
\hline Overload factor & $\mathrm{K}_{\mathrm{o}}$ & 1 & 1 & 1 & & & \\
\hline Size factor & $\mathrm{K}_{\mathrm{s}}$ & 1 & 1 & 1 & & & \\
\hline Load distribution factor & $\mathrm{K}_{\mathrm{m}}$ & 1.3 & 1.3 & 1.3 & & & \\
\hline Rim thickness factor & $\mathrm{K}_{\mathrm{B}}$ & 1 & 1 & 1 & & & \\
\hline Geometry factor for bending strength & $\mathrm{J}$ & 0.36 & 0.38 & 0.36 & & & \\
\hline AGMA bending strength $\left(\mathrm{lb} / \mathrm{in}^{2}\right)$ & $\mathrm{s}_{\mathrm{at}}$ & 75000 & 75000 & 75000 & & & \\
\hline Stress cycle factor for bending strength & $\mathrm{Y}_{\mathrm{N}}$ & 1 & 1 & 1 & & & \\
\hline Temperature factor & $\mathrm{K}_{\mathrm{T}}$ & 1 & 1 & 1 & & & \\
\hline Reliability factor & $\mathrm{K}_{\mathrm{R}}$ & 1 & 1 & 1 & & & \\
\hline Safety factor for bending strength & $\mathrm{S}_{\mathrm{F}}$ & 1 & 1 & 1 & & & \\
\hline Allowable bending stress number $\left(\mathrm{lb} / \mathrm{in}^{2}\right)$ & $\mathrm{S}_{\mathrm{t}}=\mathrm{S}_{\mathrm{at}}{ }^{*} \mathrm{Y}_{\mathrm{N}} /\left(\mathrm{S}_{\mathrm{F}}{ }^{*} \mathrm{~K}_{\mathrm{T}}{ }^{*} \mathrm{~K}_{\mathrm{R}}\right)$ & 75000 & 75000 & 75000 & & & \\
\hline Face width (in) & $\mathrm{F}$ & 1.466722 & 1.121657 & 0.977927 & 1.466722 & 1.121657 & \\
\hline
\end{tabular}

